Florida International University FIU Digital Commons

$3-5-2018$

\title{
For Those About to Rock: Gender Codes in the Rock Music Video Games Rock Band and Rocksmith
}

Elisa M. Melendez

elisamelendez@gmail.com

DOI: $10.25148 /$ etd.FIDC006558

Follow this and additional works at: https://digitalcommons.fiu.edu/etd

Part of the American Popular Culture Commons, Digital Humanities Commons, Ethnomusicology Commons, Gender and Sexuality Commons, Leisure Studies Commons, Musicology Commons, Other Film and Media Studies Commons, Other Sociology Commons, $\underline{\text { Sociology of Culture Commons, and the Women's Studies Commons }}$

\section{Recommended Citation}

Melendez, Elisa M., "For Those About to Rock: Gender Codes in the Rock Music Video Games Rock Band and Rocksmith" (2018). FIU Electronic Theses and Dissertations. 3685.

https://digitalcommons.fiu.edu/etd/3685 


\title{
FLORIDA INTERNATIONAL UNIVERSITY
}

Miami, Florida

FOR THOSE ABOUT TO ROCK: GENDER CODES IN THE ROCK MUSIC VIDEO GAMES ROCK BAND AND ROCKSMITH

\author{
A dissertation submitted in partial fulfillment of \\ the requirements for the degree of \\ DOCTOR OF PHILOSOPHY \\ in \\ COMPARATIVE SOCIOLOGY \\ by
}

Elisa M. Meléndez 
To: Dean John F. Stack, Jr.

Green School of International and Public Affairs

This dissertation, written by Elisa M. Meléndez, and entitled For Those About to Rock: Gender Codes in the Rock Music Video Games Rock Band and Rocksmith, having been approved in respect to style and intellectual content, is referred to you for judgment.

We have read this dissertation and recommend that it be approved.

Benjamin Smith

Laurie Shrage

Nelson Varas-Díaz

Guillermo Grenier, Major Professor

Date of Defense: March 5, 2018

The dissertation of Elisa M. Meléndez is approved.

Dean John F. Stack, Jr. Green School of International and Public Affairs

Andrés G. Gil

Vice President for Research and Economic Development and Dean of the University Graduate School

Florida International University, 2018 


\section{DEDICATION}

To my family. To Evan.

This is all your fault. 


\section{ACKNOWLEDGMENTS}

This dissertation, much less the research that supports it, would not have been possible without the support, guidance, kindness, and patience of countless people. To my family, who first instilled a love of both music and video games in me from a very early age - this is all your fault. To Evan, who helped me make music of my own. To Robert Loggia Baratheon, First of His Name, for laying on every book, iPad, laptop, and scrap of paper and imbuing it with your magical essence (and hair).

To Dr. Guillermo Grenier, who lit the initial torch that sent me down this long and winding path. Thank you for your patience. We did it!

To the QLC crew: for 90s compilations, for that interview with Moira Quirk, for Jackbox parties, for leaves of brown and skies of gray, for generally keeping me sane. You're the best friends anyone could ever ask for.

To the developers at Harmonix and Ubisoft, and to the players interviewed: thank you for letting me look into your world. To Gearbox, thank you for letting me be a part of yours.

Finally, thank you to Janis, Grace, Rita, Ann, Pat, Lita, Freddie, Gwen, Lisa, Shirley, Amy, Hayley, Morgan, Anita, Brenda, and countless others for inspiring me to use my voice. 


\section{ABSTRACT OF THE DISSERTATION \\ FOR THOSE ABOUT TO ROCK: GENDER CODES IN THE ROCK MUSIC VIDEO \\ GAMES ROCK BAND AND ROCKSMITH}

by

Elisa M. Meléndez

Florida International University, 2018

Miami, Florida

\section{Professor Guillermo Grenier, Major Professor}

This dissertation explores gender codes within the intersection of two American pop culture staples, video games and rock music, by conducting a feminist analysis of two video games (Rock Band and Rocksmith). Both video games and rock music have had their share of feminist academic critique: Musicologists point out how lack of canonical inclusion, gendered attitudes towards instruments, and messages from supporting media create an unwelcome environment for women to pursue a rock music career. Game studies scholars have examined similar attitudes, including a lack of women represented in both the video games and the studios that create them.

Through a mix of creator and player interviews, participant observation, content analysis, and autoethnography, I look at the intersection of these two literatures (the rock music video game) to see how gender is hard-coded into the game, and what opportunities, if any, exist for subversion of societal and industry gender norms. Through not just looking at the game as text, I present a more "thick description" of a video game that takes into account the creators of the games, the players that play them, and a researcher that occupies multiple identities within the space. I argue that, in an effort to 
replicate an authentic rock musician experience in a video game, Rock Band and Rocksmith often replicate a lot of these gendered messages. The games' text and set list emphasize a male-centric rock music canon. Rocksmith's original whiskey-soaked visual design and marketing skew heavily towards an older male demographic. However, resistances to these codes exist in both the players who defy expectations by showing up to perform and compete, as well as the creators who actively work to make these games more inclusive via changes to future games as well as inclusive hiring practices, marketing, and music sourcing (with varying degrees of success). 


\section{TABLE OF CONTENTS}

CHAPTER

PAGE

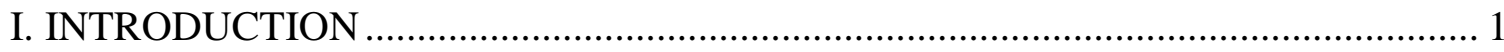

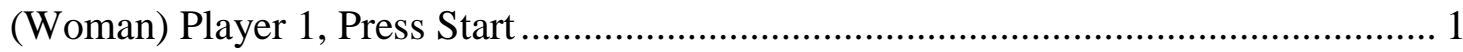

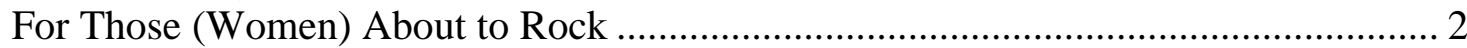

Plastic Guitars, Real Implications ........................................................................... 3

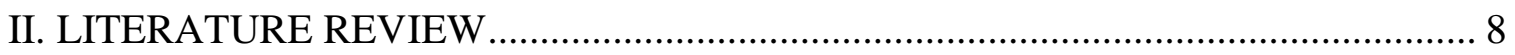

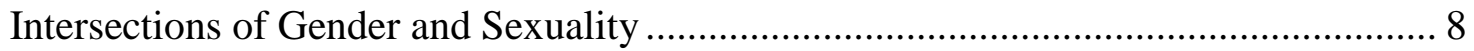

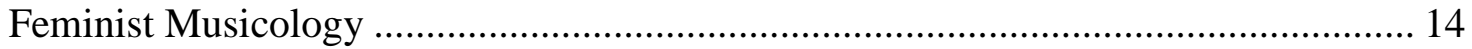

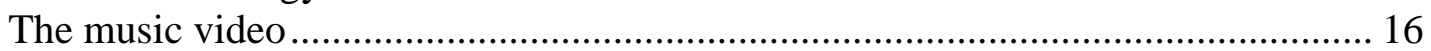

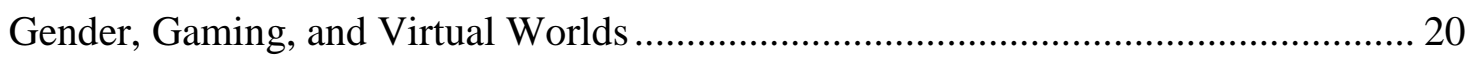

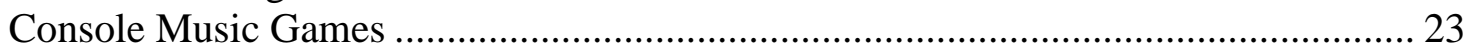

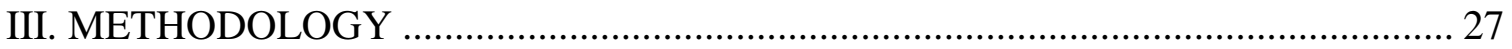

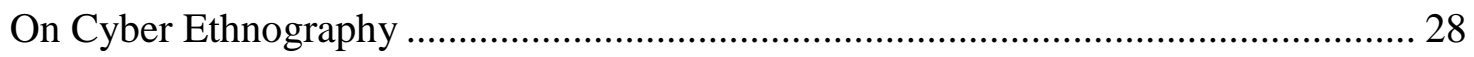

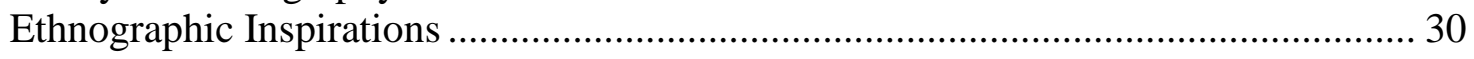

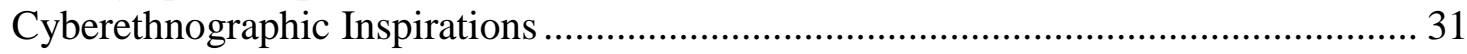

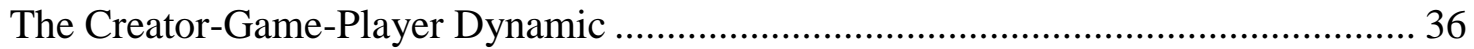

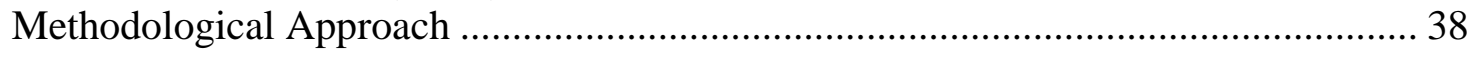

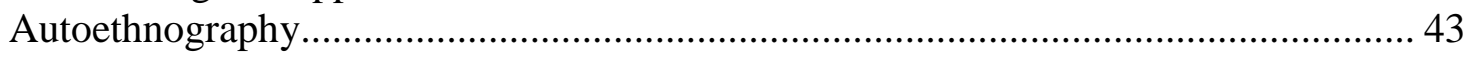

IV. THE ROCK DOC: AN AUTOETHNOGRAPHY …………................................. 44

Prologue: Putting the Rock in RockDoc................................................................ 44

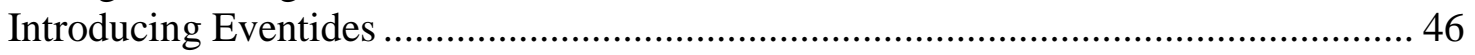

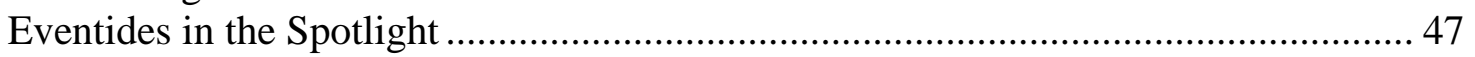

Eventides, the Frag Doll Cadette ........................................................................... 55

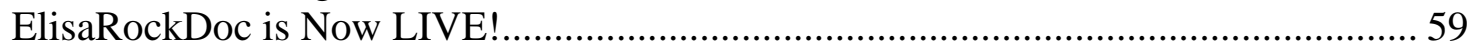

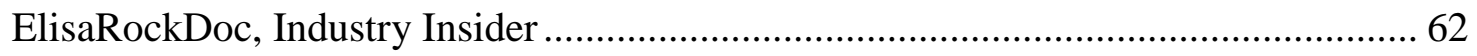

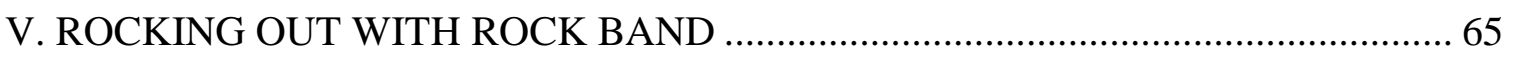

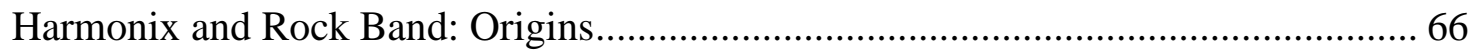

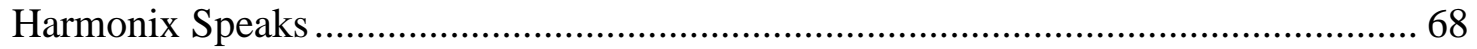

Rock Band: The Set List ............................................................................... 74

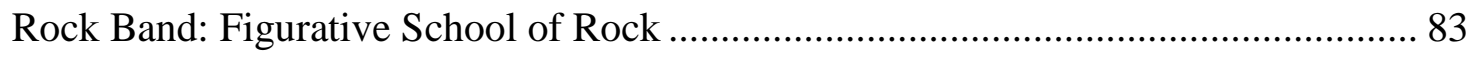

Playing at Performance ……………………………........................................... 94

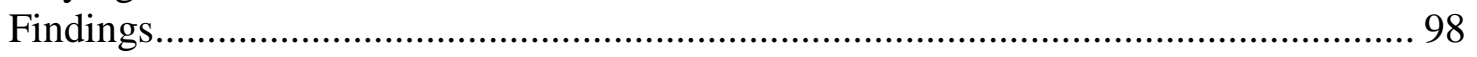

VI. ROCKSMITH: THE NEXT STEP...BUT IS IT PROGRESS?.............................. 102

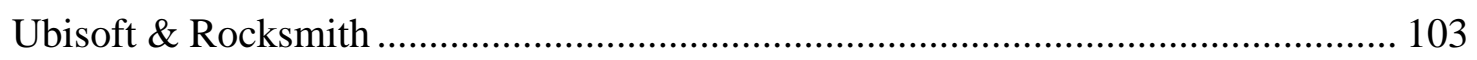

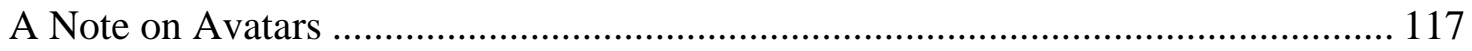

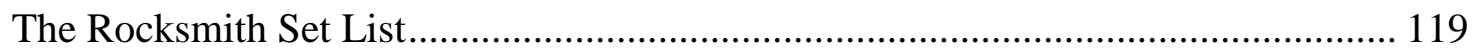

Becoming a Guitar Player...Through Swag .......................................................... 123 
For Those About to Rock, Check Out Our Booth 124

Findings.

VII. THE PLAYERS: BUTTONS, STRINGS, AND A FLIP OF THE HAIR............. 132

Our Avatars, Ourselves ...................................................................................... 132

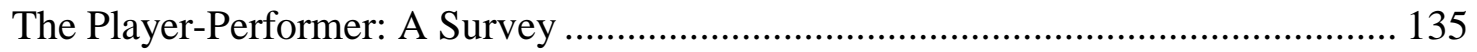

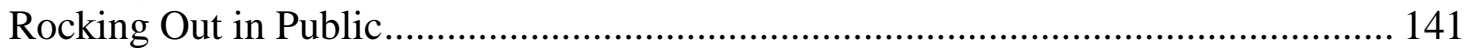

When Players Compete: An Interview ................................................................. 145

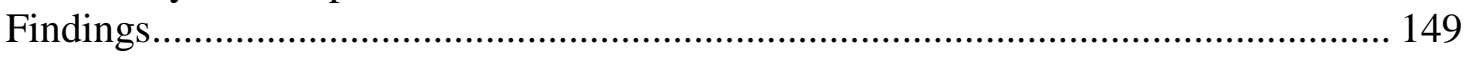

VIII. DISCUSSION, LIMITATIONS, AND CONTRIBUTIONS ............................ 152

Rock Band and Rocksmith: Gendered Cover Versions ........................................ 152

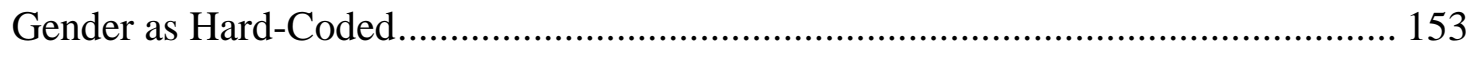

Subversion in Song? .................................................................................... 155

Verse, Chorus, Solo, Double Chorus, Fade Out: Subverting Expected Formulas...... 156

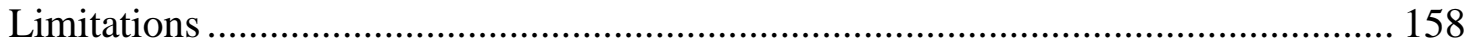

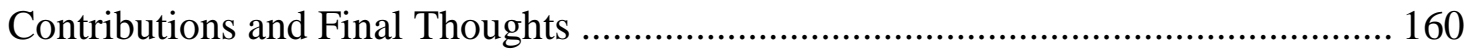

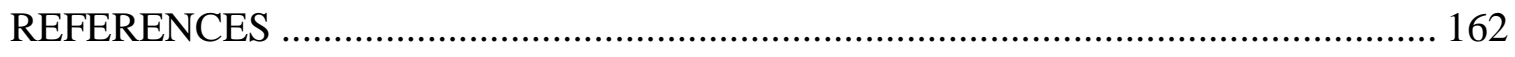

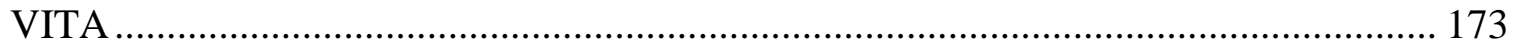


FIGURE

\section{LIST OF FIGURES}

Figure 1: Eventides the Avatar on Xbox Live. Retrieved from

https://account.xbox.com/en-US/CustomizeProfile in October 2011

Figure 2: Sending Eventides to the kitchen. Retrieved from Xbox Live in

October 2011. 53

Figure 3: Sending Eventides to the kitchen. Retrieved from Xbox Live in

October 2011 53

Figure 4: Asking for it. Retrieved from Xbox Live in October 2011.............................. 53

Figure 5: A delightful greeting. Retrieved from Xbox Live in October 2011.................. 54

Figure 6: Really painting a picture. Retrieved from Xbox Live in October 2011............ 54

Figure 7: GameCrush.com "booth babes" at PAX East 2010. Retrieved from https://cdn.gamerant.com/wp-content/uploads/GameCrush-girls-1.jpg May 1st, 2017... 58

Figure 8: A typical view of the video game broadcasting site Twitch.tv. Retrieved from twitch.tv/elisarockdoc on November 10, 2017.

Figure 9: Researcher as performer. Retrieved from twitch.tv/elisarockdoc

May 1st, 2017

Figure 10: The Rock Band start screen. Screenshot from Rock Band.............................. 66

Figure 11: Real-life sponsors for a virtual band. Screenshot from Rock Band

May 1st, 2017 71

Figure 12: Chi-square results when looking at Female Presence, instrument use, and song difficulty (Retrieved from XLSTAT November 2011). 76

Figure 13: Representation in the Rock Band set list. Image rendered in Excel. 79

Figure 14: Distribution of difficulty levels in Rock Band in number of stars. Image rendered in Excel.

Figure 15: Distribution of difficulty levels among songs which do not feature women musicians. Image rendered in Excel.

Figure 16: Distribution of difficulty levels among songs in the overall Rock Band song list which feature a woman musician. Image rendered in Excel. 82 
Figure 17: Loading screen text with game tips. Screenshot from Rock Band.

Figure 18: A makeup option, Stardust, which has the exact same look, name, and descriptive text for both male and female avatars. Screenshot from Rock Band.

Figure 19: Women's corduroy pants. Four Cords: Four chords are better than three in these perfectly worn corduroys.

Figure 20: Men's corduroy pants. "Worn Cords: They say you only need to know three chords. How many do you know? These cords will let them know what a virtuoso you are."

Figure 21: A Line 6 shirt unlocked through successful guitar play............................. 93

Figure 22: Being on the road is hard. Screenshot from Rock Band. ............................. 96

Figure 23: You can select and preview your rocker's attitude, which dictates your actions onstage. Screenshot from Rock Band.

Figure 24: The 2011 Rocksmith intro, featuring a headless leather-clad guitarist in a darkened room. Screenshot from Rocksmith.

Figure 25: The Rocksmith main menu, which outlines your point progress and labels you based on your experience. I reached "International Headliner" status. Screenshot from Rocksmith.

Figure 26: The headless leather-clad instructor, in darkened room. Screenshot from Rocksmith.

Figure 27: The Rocksmith 2014 start screen. The "Remastered" comes from a reworking of the game that released in Fall 2016. Screenshot from Rocksmith 2014... 111

Figure 28: The Rocksmith 2014 main menu. Light coming through the window plus a brightly-colored wall accent made of guitar pedals. Screenshot from Rocksmith 2014. 112

Figure 29: The new lessons menu, featuring a woman instructor as the thumbnail for the guitar-holding lesson. Screenshot from Rocksmith 2014.

Figure 30: Lessons occur in a brighter room, with the instructor even wearing brighter colors. Screenshot from Rocksmith 2014.

Figure 31: In the 2011 version, you practice in the same darkened room as the intro. Screenshot from Rocksmith. 
Figure 32: Performing in front of a crowd. Screenshot from Rocksmith.

Figure 33: The fog clears to reveal a cheering audience-if you do well enough.

Screenshot from Rocksmith 2014.

Figure 34: Your success in Rocksmith determined by points. Screenshot from

Rocksmith.

Figure 35: Rocksmith 2014's scoring, based on percentage. Screenshot from

Rocksmith 2014.

Figure 36: Gender makeup of Rocksmith artists. Image rendered in Excel.

Figure 37: Breakdown of women in Rocksmith. Image rendered in Excel.

Figure 38: A common Rocksmith giveaway on their livestream. Screenshot from twitch.tv/rocksmithgame.

Figure 39: The researcher (center) working the Rocksmith booth at PAX West 2011. . 125

Figure 40: The researcher demonstrating Rocksmith in Capitol Hill (Seattle, WA).

Note the branded guitar picks used for swag on the table to the right.

Figure 41: Racial/ethnic breakdown of respondents. Image rendered by

SurveyMonkey.

Figure 42: Gender breakdown of respondents, with categories already problematically out of date. Image rendered in SurveyMonkey.

Figure 43: Embellish the sound of the song through whammy bars or effects to make the song your own. Screenshot from Rock Band. 


\section{INTRODUCTION}

(Woman) Player 1, Press Start

I sign onto my Xbox and join a party with other members of my all-woman

gaming community. We decide on Blur, a high-powered racing game, as the night's game

of choice. We enter a lobby, wait for a race, and chat amongst ourselves. A slurred, low

voice from the opposing team cuts through:

"Hey, female girl."

We laugh to ourselves. The sentiment is nothing new, but the delivery is novel.

"Hey, female girl. Where you from? What's your name? You single?"

Our collective would be referred to as "Hey, female girl" by the opposing team for the rest of the night.

Video games are an increasingly important form of mainstream popular media worldwide. The Entertainment Software Association, in its most recent annual report, states that $67 \%$ of U.S. households own a device used to play video games, and that $54 \%$ of the most frequent gamers feel that video games provide more value for their money than other media such as music or movies (ESA 2017). With video games becoming more of a presence in households (and outside of them, as evidenced by last year's smartphone phenomenon Pokémon GO), it becomes increasingly important to turn an academic eye towards the media we consume and be mindful of potential effects on the people who engage with it. Academia has, in fact, turned its attention to this growing industry in recent decades, including feminist critiques, which figure prominently in my research. Even though the Entertainment Software Association (ESA) reports that women 
age 18 or older are a greater portion of the gamer population than boys under 18 , many mainstream video games as well as the marketing surrounding them often rely on heteronormative, sexist tropes (ESA 2017). Feminist critiques of video games overall have commonly focused on the violent and over-sexualized characters and questions of feminine agency in adventure games and first-person shooters (Dietz 1998, Burgess et. al. 2007, Miller \& Summers 2007, Soukup 2007). These studies conclude that video games are gendered spaces in which male characters possess the majority of the agency and exert their agency in the form of violence, while female characters generally fall into four categories with few exceptions: the damsel in distress, the barrier to progress, or pure eye candy (Dietz 1998). While my personal interests lie in video games of all genres (and I now work for a video game developer and publisher that produces games in multiple genres), the focus of this research is the rock music video game, exemplified by games such as Rock Band and Rocksmith. The origins of the interest are from my own experience as both a woman who plays games and a woman who plays rock music.

For Those (Women) About to Rock

I'm the lead singer of a rock band getting ready for a performance. An hour before our set starts, I head to the bar to grab bottles of water for the rest of the band. A man sits down next to me and eyes my frame, clad in dark jeans, lace-up boots, and fishnet top. [I wonder if this outfit was a mistake.] He places his hand on my wrist, adorned in a studded bracelet mere inches from a hand containing an engagement ring. 
[I wonder why it matters that I was engaged.]

"Are you with someone in the band, sweetheart? Don't break my heart now." I glower at him behind layers of black eyeliner. "I'm IN the band."

Similar to video games, popular music is subject to the same feminist academic scrutiny. Feminist critiques of popular music (Bayton 1997, Garr 1992, McClary 1993, Whiteley 2000) have focused on gendered performance in music videos, stage performances, and lyrical content. Throughout rock music history in the US, women have had notable boom periods as musicians. However, they are still seen as a novelty and not as "authentic" as their male musician counterparts, neither in technical proficiency or in lifestyle (Clawson 1999, Garr 1992 Whiteley 2000, etc.). Instruments themselves are particularly gendered, with women being considered more naturally adept at being vocalists while their prowess in other instruments is challenged and called into question.

Plastic Guitars, Real Implications

I start playing Guitar Hero: World Tour, ready to take on the game with my vocal prowess. As a singer in a rock band, playing the game is a great way to practice. My husband and guitar player chooses to play the drums instead - it's not as authentic to play a plastic guitar, and it's nice to play something else for a while. We select a song to play together, and wait for the loading screen to give us a tip on how to play the game, or a fun fact about the band we selected.

A tip flashes on the bottom third of the screen: "When in doubt, blame the singer's girlfriend!" 
The rock music video game serves as an intersection between video game and pop musicological literatures. Games such as Rock Band and Rocksmith produce a discourse that heavily references the music industry. At the same time, these games are part of a wider gaming industry discourse with game design conventions that are highly genderdisparate. Another significance of this genre of games is in their reach. Many of the games that find themselves within game studies literature can be considered mature titles, rated for players over 17 years old. Music games, however, are typically considered as being acceptable for all ages by the Entertainment Software Ratings Board, opening up a wider and younger audience to the discourses in question. Video games are an increasingly mainstream agent of socialization, and the rock music video game has a great deal to tell younger generations about what it is to be in a rock band or sling a guitar like a hero - and what opportunities are available for women who decide to pick up a guitar or a game controller.

These games are important for analysis because of their unique placement on the wide spectrum of possible interactions between player and game. Rock Band uses instrument peripherals made to resemble performance-grade instruments. Rather than sitting and pushing a series of buttons on a classic controller, a player can move the guitar, bang on the drums, or scream into the microphone to score more points. Rock Band has been embraced as a game to play in groups, replacing traditional karaoke at bars and Rocksmith goes one step further by having players plug in any real guitar and learn how to play songs, blurring the lines between game and musical performance. This unique player relationship makes these games particularly suited for discussions of agency and gender identification and performance. 
The underlying theory driving this research is that both Rock Band and Rocksmith are both a product of the gaming industry and the rock music industry, which work in conjunction to present a caricature or "cover version" of the "real" rock band experience. A "cover version" of a song will often use the lyrics and general melody of the original song and even some recognizable instrumental moments, but it becomes a new recording or performance due to changes in instrument usage, arrangement, or vocal style. I argue that these games represent a "cover version," creating a new version of a rock band experience out of the merger of recognizable rock music culture and game design traditions. By bringing in recognizable rock music culture, the games tend to bring other recognizable baggage along with it, including the gender disparity inherent in the industry: a relative dearth of female talent, with the presentation of this talent sexualized through clothing and performance. This leads to a presentation of female rock musicians as novelty and "Other" versus the unmarked white male rock musician category.

For the purposes of this dissertation, I decided to focus completely on gender as an aspect of rock performance in order to perform a deeper dive that was relatively lacking in existing research on gaming and rock music. I recognize that, by focusing on gender, I run into the issue of reifying a monolithic version of gender that does not take intersectionality into account. I risk creating my own version of rock music culture that imitates the mainstream: lacking in of women of color, with a cursory nod to transgender identity in the margins (not to mention the class issues inherent in access to the technology required to create music, create games, and play them. I discuss the limitations of such an approach in a subsequent chapter, but I also assert that significant 
gaps in the pre-existing research on gender, rock music, gaming, and their intersections can be filled (in part) by this project.

The objectives of my research are to answer the following questions:

1. How are Rock Band and Rocksmith gendered? How do they emulate or subvert gendered music or gaming discourses?

2. How are gender norms hardwired into the game itself?

3. What possibilities exist for the subversion of gender norms? Where do these resistances occur (solo performance, small groups, public play/competitions)? Are they effective resistances? Why/why not?

In order to help answer these questions, I employ a mixed methods approach, including quantitative analysis of songs available in the games, a discourse analysis of avatar customization options, interviews with creators, player surveys, and participant observation at events. I also found it important to include an autoethnography chapter, as I find it necessary to couch this research in my experience as both a musician and a "gamer," and it is necessary to discuss the ways in which these experiences affect the research and vice versa.

Through the use of these methods, I find that Rock Band and Rocksmith do in fact both emulate and subvert existing music and gaming discourses. These are discourses that paint women as rare exceptions to a vast male-dominated canon, the reasons for which are couched in outdated linkages between technical prowess (or lack thereof) and gender. The games both reify these discourses and subvert them through design, intent, and through their marketing efforts (which aid in their own formation of attitudes surrounding the game and influence their purchase). I do find, however, that the creators 
are aware of changing attitudes surrounding gender and video games and use narrative elements as well as user interface design in order to attract a wider audience. Players are also capable of subverting gender norms through public play through the appearance of women on male-dominated stages and employing rock performance that is raw and aggressive, even as they reify rock performance tropes during their play sessions, and even as opportunities to do so become limited as music games wane in popularity. Players also show an increased awareness of gender issues in gaming, although they see less occasion for issues such as harassment in the music gaming genre. In this way, rock music games present a fascinating and malleable point of intersection between gender performance and music performance. 


\section{LITERATURE REVIEW}

\section{Intersections of Gender and Sexuality}

Erving Goffman's (1959) dramaturgical model for social interaction, along with Judith Butler's performative theory of gender $(1988 ; 1990 ; 1993 ; 2004)$ are particularly instructive, along with other queer and feminist thinkers which corroborate notions of social performance. The metaphors produced by conceiving gender and other sociallyconstructed identity markers as performatives lend themselves well to a study of rock music-themed video games, where so much of its play is based on referential rock music stage performances.

Erving Goffman's dramaturgical model of social interaction (1959) presents interaction as a performance, with performers embodying a set of roles, language, and costuming depending on the context of the performance. Thinking through the performance metaphor involves not just words recited as script, but elements such as props and costuming. Looking at music gaming, it is important, then, to look at the instrument peripherals and costuming that avatars can wear in addition to the performances themselves.

Goffman provides insights on gendered performances in particular: his discussion of an arrangement of the sexes (1977) posits gender as a "sex-class" or "sexual subculture" which has imposed on it a series of actions, feelings, beliefs, and acceptable appearances (328). In a startlingly prescient moment, Goffman points to the possibility of fictional creations (in the case of this study, the virtual worlds of video games) of being a mirror of real life gender scripts and stereotypes: 
And should we leave the real world for something set up as its fictional alternative, a science fiction cosmos, we would find that here, too, males engage in the executive action and have females to help out in the manner of their sex. Wherever the male goes, apparently, he can carry a sexual division of labor with $\operatorname{him}(315)$.

This passage is particularly central to my analysis in my endeavors to find out to what degree men and women carry these sexual divisions of labor into game development and in the game discourses themselves.

There is much use in Goffman, but I would not make a strict distinction between his "frontstage" and "backstage" selves, which imply that there may be an ultimate, true self separate from its performance of it (1959:22). The self - the gendered self in particular - is constantly in the process of construction from act to act, regardless of whether or not there is an audience to observe the performance.

Feminist theorist Judith Butler's discursive model makes gender central to the performance metaphor, defining gender as constructed through "the stylized repetition of acts through time (1988:520)." For Butler, gender is not essential, and is nothing without the actors who perform it: "gender is an act which has been rehearsed, much as a script survives the particular actors who make use of it, but which requires individual actors in order to be actualized and reproduced as reality once again (1988:526)." Gender is constructed not just by language, but by bodily acts as well (2004:198), which lends importance not just to the study of textual representation or language used in my 
research, but to the bodily performance of both players and avatars, which carry their own clues as to the gender discourses which shape them.

Butler is predated by West and Zimmerman (1987), who brought forth the concept of gender as being constructed through "a complex of socially guided perceptual, interactional, and micropolitical activities that cast particular pursuits as expressions of masculine and feminine "natures' (126)" in opposition to previous conceptions of gender as a more statically constructed role which is put on rather than as a constant state of becoming. Butler moves this concept forward by entrenching the concept of gender within a performative model that can serve to subvert dominant discourses.

Martha Nussbaum (2000), a critic of Butler, holds that Butler's advocacy of subversive performative acts does not go far enough in defining whether these acts can be positive or negative, or what exactly should be subverted, and how. Nussbaum also contends that "resistance is always imagined as personal, more or less private, involving no unironic, organized public action for legal or institutional change (10)." While Butler does not provide exact prescriptions for subversions, the concept of everyday performative acts as constitutive of gender rather than the biological inevitability of it is still an important tool (among many others) with which to trim away at the systemic hegemony of gender as static category. To wit, just the work of having shown the social constructedness of gender could be enough for individuals to take up the work of individual action, but I agree that more could be done to show how those individual actions can turn into collective action, so that the answer awaits the reader when they have their "what's next?" moment. 
In applying Butler, as Lise Nelson (2014) critiques, one must be wary to not apply performativity exclusively from her early Gender Trouble and Bodies That Matter perspective, which leaves little room for agency in the production of subversion. In applying Butler to music games, it would be tempting to see coded gender interaction with a plastic guitar (or real one) through the lens of either completely unintentional mimed repetition that concurrently reproduced gender scripts. An equally (if not more) heinous application of Butler would be to inject what Nelson calls "a voluntaristic understanding of performativity" that inserts "agency into performativity that [is] at odds with Butler's steadfast rejection of the idea of a 'doer behind the deed' (108)." Rather than take Early Butler's performativity at face value, the study aims to put Butler's performativity metaphor in conversation with such agent-centric theorists as Goffman, as well as place Butler in conversation with herself: To what extent does this classic performativity sans agent mirror the designed illusory choice inherent in video games? To what extent does the Rock Band/Rocksmith performance call into question Butler's agent-less performativity and provide opportunities for subversion of these designed elements?

What is a performance without a stage or an audience? Another element of gender performance is the stage or setting in which it takes place as well as the audience which either recognizes the performance or doesn't. Rock stages and the gaming industry (as well as the games and music which are their products) are places where heterosexuality is consistently naturalized through performances which often link "masculinity to activity and femininity to passivity,” (Hubbard 2000:197). On the physical stage of the music performance, the phallic guitar is placed in a position of activity, typically by a male 
performer. Women in rock, with notable exceptions, have been relegated to support instrumentalist or groupie rather than guitar hero. In virtual worlds, male heroes are put in positions of activity predominantly through violence, while women are often passive princesses in need of rescue - if they appear in the virtual world at all. These games rely on authentic performances of both video game design expectations and rock music culture in order for the audience to buy into the fantasy being sold.

The research looks at ways in which the corporeal stage and the virtual stage of the video game (and the interactions between the two stages which occur during play) are platforms for both subversion and reification of normative hegemonic discourses. Insomuch as rock performance and interactive virtual play involve bodily movements upon these stages, hegemonic essentialist discourses which make references to the physical body, 'serve to naturalize what is in fact social difference...[and] references to the biological body are seen to reinforce patriarchal claims that women are naturally incapable of certain kinds of action (Longhurst 1997:489)," such as playing certain instruments over others or being adept at gaming.

Using Mulvey's (1975) research on visual pleasure and cinema, one can make the argument that the myriad visuals of both the music and gaming industry are often created in the service of a male gaze. As seen in the following sections, ads in guitar magazines which showcase women as ornaments for instruments rather than as performers themselves, or first-person video game points of view which privilege the vision of a typically male protagonist: "Traditionally, the woman displayed has functioned on two levels: as erotic object for the characters within the screen story, and as erotic object for 
the spectator within the auditorium"- or living room, in the case of video games (1975:11).

There are also new avenues through which queer research and activism can be applied to the gaming industry where questions of marginalization are only recently becoming a part of the gaming culture's conversation. Members of the game-playing population that are marginalized through gender, sexuality, etc. would do well in looking at Berlant and Freeman's (1992) examination of Queer Nation as an example for activism, specifically the Queer Nights Out, where same-sex couples deliberately occupied straight bars and restaurants to confront "customary segregation (162)." Only a few substitutions need to be made to Berlant \& Freeman's explanation of the strategy's effectiveness to see how it could benefit gamers:

Demonstrating that the boundedness of heterosexual spaces is also contingent upon the (enforced) willing ness of gays [and women, and people of color] to remain invisible, queers are thus using exhibitionism to make public space psychically unsafe for unexamined heterosexuality [and homosociality] (162).

A Gamers' Night Out would consist of female, transgender, and other marginalized groups of gamers to open up the microphone channel, speak freely in multiplayer games, and construct their avatars to either reflect themselves or perform some sort of virtual gender-fuck en masse. Here, literal fun would carry political weight, reclaimed by those previously silenced, as per theorist Sarah Ahmed: "Spaces are claimed through enjoyment, an enjoyment that is returned by being witnessed by others...to display 
pleasure through what we do with our bodies [or our controllers] is to make the comforts of heterosexuality less comfortable (2004:165)."

\section{Feminist Musicology}

Feminist musicology refers to the scholarship of music, its creation, and its history from a feminist perspective. Feminist musicology literature shows how both musical instruments and mediums such as the music video carry meanings that often reflect overarching hegemonic discourses, such as those surrounding gender and race. Instruments

Women in rock music are overwhelmingly perceived as vocalists rather than as instrumentalists. However, when they do play an instrument, women are more often bassists than guitar players and drummers (despite the guitar being the signature instrument of the rock genre). Mary Ann Clawson (1999) examines this overrepresentation of women on bass and explains it, through interviews with local musicians. The instrument's ease, link to feminine traits (such as support/care work), and place in the gendered hierarchy of instruments in rock music are cited as reasons driving women towards the bass : "Everyone who discussed it not only agreed that the bass is perceived as the rock instrument that can be learned most quickly but assumed that ease of learning would be especially important for women (199)," as they do not have the benefit of numerous role models or early adoption as their male musician counterparts may have.

Aspiring women rock musicians are often denied the years of teenage apprenticeship and skill acquisition experienced by male counterparts (Clawson 
1999:200). This is especially true when considered in conjunction with Mavis Bayton (1997)'s assertion that guitar magazines and other sites promote rock music instruments using women as eye candy rather than as players themselves. Bass players are often in short supply due to its lack of attractiveness vis-à-vis the rock instrument hierarchy. The bass is also gendered as performing the role of emotional support, a role that women are seen as naturally more adept in performing. The bass is also linked with rhythm and other musical intangibles such as "groove" and "feel," which are also perceived as more naturally in tune with the feminine. When this is combined with its perceived ease of learning, the bass is a quick way for women to gain entry into a band. Clawson uses Reskin and Roos's (1990) “queuing theory” to explain how this could be: Women first make strides in gaining access to male-dominated fields by entering occupations that have a shortage of suitable' male workers (1999:198). The bass has a lower prestige level than the guitar, the signature instrument, and is therefore often met with disinterest. Disinterest and rejection by men lead to more opportunities for women (although, relatively speaking, those opportunities are still few and far between).

When women do choose to pick up the electric guitar, their choice can be met with resistance. Mavis Bayton (1997) focuses on the relationship between women and the electric guitar, as well as the ways in which the guitar's masculine gendering is reflected through guitar magazines, performance spaces, and guitar stores. In her study, she performs a content analysis of women's representations in guitar magazines as well as conducts interviews with musicians. The review of guitar magazines yielded a very small percentage of women (only two women pictured in an August 1996 issue of Guitar). The women that were present in her review of mid-90s guitar magazines were more likely to 
be pictured as stereotypes, scantily-clad, draped over amplifiers, and barely holding any guitars at all (unless they're the "softer," better-suited acoustic guitar).

A common theme among Bayton's interviews with musicians is the denigration of women by their musical peers, concert attendees, and exploitative record labels. Guitar shops maintain male homosociality in hiring practices, making them foreign territory for female guitarists: "trying out the equipment was akin to being on trial; they [are] scared of...being 'put down' or laughed at," while more experienced female musicians risk “condescension or simply being ignored (42)." Bayton asserts that rock music is no different than any other male-dominated field in which the men turn to harassment at the infiltration of women, a harassment that is based on both "positive" bodily perception and negative skill perception: "female guitarists are expected to be sexy and incompetent (47)." This is true of both rock music and video games, where women are often harassed if there is even a hint of gender presentation.

\section{The music video}

Feminist examinations of music videos, which gained traction with MTV's debut in 1981, have typically been an exercise in rooting out misogyny where it lies, regardless of the genre of music being examined (Dibben 1999; McClary 2000; Mercer-Taylor 1998; Sommers-Flanagan 1993, etc). That being said, both Robin Roberts (1990) and Rana Emerson (2002), find music videos to be avenues where alternate gender discourses can be displayed.

Robin Roberts (1990) champions the use of these popular culture mediums as a source of empowerment not to be easily dismissed. The dominant feminist critique, 
which once focused on decrying the sexist music video, further separates popular music (and thus, popular culture) from a status of high art with little merit or reason for scholarly study. She states,

To reduce popular culture to its worst stereotype and to dismiss it as a site of feminist inquiry is to oversimplify a complex subject. More importantly, to neglect popular culture is to ignore...the moments of resistance and instances of female triumph in an arena that reaches a far wider audience than the most popular feminist analysis (2).

Roberts was one of the first voices to rise up in favor of music video as a medium with feminist potential. In her 1990 article, she describes videos by female artists that subvert hegemonic music video gender discourses. The video for pop/rock singer Pat Benatar's "Sex as a Weapon," for example, deals with the idea of objectification and sexism directly in its lyrics. The combination of lyrical content and visual interpretation is a powerful mix: By playing with sexist images and rebuking them (including images from her own previous album covers), Benatar shows how the medium that is used as advertisement can also “operate... self-consciously and, most importantly, politically (10)."

Rana Emerson (2002) would provide another nuanced example twelve years later, as she sees hip hop music videos as avenues for both controlling Black women's expressions of sexuality and providing them methods of resistance:

While most music videos, including those of some Black women performers, exacerbate the exploitations of the Black woman's body and perpetuate 
stereotypes of Black womanhood, Badu, Elliot, and Hill depict themselves as independent, strong, and self-reliant agents of their own desire, the masters of their own destiny (116).

Emerson places importance on the camera's gaze, in a send-up of Mulvey (1975), and uses the point of view of the camera to find emergent patterns of social constraint. The first pattern is in the body shape/size of the women present in the videos (not just the performers), who are very similar. Another pattern is in the one-dimensionality of womanhood: non-normative beauty representations and alternative gender and sexual performances are often conspicuously absent, and women are mostly represented as objects of the male gaze, particularly in the form of a patriarchal figure (a producer or rapper mentor) who is responsible for the female performer's success (Emerson 2002:123-4).

Countering these representations does occur, albeit not as often as normative representations of Black womanhood. As a show of agency, Blackness is reaffirmed in a positive context and as synonymous for "strength, power, and a positive self-identity (125)." The voice, literally and figuratively, is used to illustrate the active role of Black women in music videos—lyrics and the video's themes can emphasize both "speaking out and speaking one's mind (126)." These contradictory messages present in contemporary hip hop music videos displays a tension in the negotiations of Black womanhood, and a consistent struggle between affirmation of self and an affirmation of hegemonic gender roles. 
One more examination of music videos to consider is Den Tandt's (2004) look at the relationship between music video and the homosocial masculinity of classic rock. Den Tandt contends that the advent of the music video brought with it an uneasy relationship between rock performers and the medium. Classic rock, according to Den Tandt (and contrary to Kania's recorded product ontology) privileges 'realist' concepts of musical performance i.e. live versus recorded, 'authentic' musicianship versus synthesized instruments. MTV, on the other hand, privileges special effects, dancing, and even cross-gender images, often characterizing it as a more feminine aesthetic (141). In fact, one such music video he examines, Dire Straits's “Money for Nothing," explicitly set up this contrast "between supposedly real (masculine, manual) and fake (feminized, techno-generated performance (142)." This contrast further illuminates how enmeshed performance, instrumentation, and masculinity are in the hegemonic rock music discourse.

The work of Emerson and Den Tandt is a reminder that social performances need not be limited to femininity, but also involve performances of masculinity and race. While race is not necessarily the central focus of my work, the interconnectedness of race, gender, and sexuality cannot be ignored in my topic, especially concerning a genre of music that had its origins in African-American music but was whitewashed into mainstream palatability. To whit, scholars such as Amber R. Clifford-Napoleone (2015) cover the possible harm in focusing exclusively on the masculine and heteronormative in rock music and particularly heavy metal: one risks reifying masculinity and heteronormativity in the process, instead of destabilizing definitions of masculinity and literally queering the rock music space: 
Gendered and sexualized queer styles, performances, and self-identified queer fans have always operated in the heavy metal landscape, the reality behind the mirage of masculinity...it is masculinity (emphasis mine) that is the mirage (12).

Based on closer readings of both music and gaming literature, as well as my own insider perspective, the practice of rendering the mirage of masculinity a reality and not acknowledging the always-there queer fans of video games and popular music is alive and well. I acknowledge my own risk in adding to a literature that does not effectively queer either industry, but I have chosen to perform a deeper dive into one particular facet of this lesser-explored genre of games.

\section{Gender, Gaming, and Virtual Worlds}

A significant amount of gender and gaming/virtual worlds literature takes the form of content analyses of popular video games in order to draw conclusions about gender portrayals and possible effects on socialization. Common subjects of analyses are the characters (Miller \& Summers 2007, Burgess, Burgess, and Stermer 2007, Beasley 2002) and a video game's narrative content (Dietz 1998) with all reaffirming the overwhelmingly sexist portrayals of both men and women as video game characters. Commonly, hyperviolence is the dominant male trait, while hypersexuality is the dominant female trait. For example, Miller and Summers (2007) reviewed gaming magazine articles and coded the gender differences among 115 video game characters. After coding for roles (hero, villain, supplemental character), attributes, and tools available to them, they found that approximately $26 \%$ of female characters were actually playable, with only $10.2 \%$ of the games explored giving a dual gender option. Their 
findings directly support the underlying assumptions inherent in this study that there is a drastic disparity in the available roles for males and females in video game appearances.

Video game covers are the subjects of analysis for Burgess, et. al. (2007). The covers are considered an important point of study as they provide the first impression in a retail environment. Coding for the prominence of the character, the implied/direct violent acts of the characters, and the body shape of the female characters, the results were dismaying. Men were the most portrayed (90\% of the covers versus $40 \%$ for the women) and the most likely to be shown in positions of direct or implicit violence. Burgess et al. define the role of violence in gender discrepancy as follows:

One could argue that the relative lack of illustrated action on the part of the female characters is actually a positive portrayal given that much of the action male characters portray is violent. Unfortunately, as video games currently exist, violence connotes power. No characters, male or female, are portrayed as central to a game by stopping violence, or offering peaceful solutions. By excluding women from the action roles, they are relegated to groupie status (427).

The physical characteristics of the human characters were the greatest show of gender disparity, as men were more likely to be coded as muscular and athletic, while the majority of female characters were categorized as "busty" or "super-busty (2007:428)." The conclusion drawn is that, even in situations in which female characters are given agency (which were few and far between), the exaggerated feminine trappings of thinness and bustiness were more important than a realistic or natural depiction of women performing a variety of active tasks. This does not necessarily mean that women characters are stuck in this position, however. Women that may not have an active role in 
one entry of a game franchise can become playable characters as the franchise unfolds, such as the case of the Uncharted franchise, which turned two of its supporting cast of women into the protagonists of the story. In light of these developments, it becomes important to focus on markers of agency and appearance as a point of reference for my research on Rock Band and Rocksmith.

Another subsection of the literature examines the effects of video games on their players, mostly through a psychological theoretical and experimental framework (Banakou 2010; Bartlett 2008; Behm-Morawitz \& Maestro 2009; Fox \& Bailenson 2009; Yao, Mahood, and Linz 2009, etc.). Although gender is not problematized and is often taken for granted in these studies, the results point to a need to move beyond the study of game-as-merely-text and instead look at linkages between game content and player perceptions.

Psychology scholars Bartlett and Harris (2008) investigate the effects of video games on players' body image and self-esteem. Using mass media research as background, Bartlett and Harris hypothesize that video games cause the same negative effects as thin female models or muscular male models do to viewers. Two studies were conducted in which men and women played 15 minutes of a game depicting an ideal body type: WWE wrestling for men, and beach volleyball for women. Negative effects on body image were reported after only 15 minutes of gameplay, showcasing the ability of video games to have effects on players' psyches. This study provides one example of the ways in which games and their effects have been studied from a psychological perspective, and where gender is not complicated or problematized. 
The following section discusses arenas in which feminist musicology and feminist gaming literature intersect, followed by their research-specific intersection: the rock music video game.

\section{Console Music Games}

Ethnomusicologist Kiri Miller (2012) has been the first prominent scholar to address a relationship between music and the digital age that takes video games into account on a large scale. Miller's perspective is that of musician insider perspective, and she provides excellent insights into the associations being made between Guitar Hero and Rock Band, and the rock genres it aims to emulate. The key difference between these rock music games and other video games is the sense of not only production, but also performance. In other video games, when you press a button on a controller to incite an action, the product of that action is not replicated in your living room. Advancements in motion controls may make it possible for a player to literally kick, punch, and slice, but the product of those actions (an injured enemy, sliced piece of fruit) does not materialize in your living room. In Guitar Hero and Rock Band (and, for my research, Rocksmith), a correct push of the button produces the music that fills the living room. In Guitar Hero and Rock Band incorrect button presses are met with silence, giving proper play the benefit of a feeling of actually making "real" music happen, further blurring the line between player and game:

This "realness" is grounded in a shared cultural assumption that music consists first and foremost of sound, so recorded music is not considered to be "fake" 
music (although live musical performance retains special status, and faked live performance therefore evokes special scorn) (103).

It is this "special scorn" that leads to detractors, often experienced musicians, to deride these games and chastise players for not playing a "real" instrument. However, the game's designers actively encourage a sort of 'buying into' of the hegemonic rock performance narrative that is reverent to the rock genre and ultimately encourages the transition into 'real' instrument through the introduction of 'pro' modes that inject more realistic playing styles—and Rocksmith's designers start off with the 'real' instrument in mind, allowing any 'real' guitar or bass to be plugged into the game.

Beyond the instrument skill, it is rock performance that is being taught, particularly in Rock Band where the guitar's movements can trigger scoring power-ups. The game directly influences a rock-referential performative identity through "the musical repertoire, archetypal rock-star avatars, a responsive crowd, a guitar-shaped controller, and physical performance hints" such as the one mentioned above, which designers admit was aimed at making the games more than just a music simulator, but a rock performance simulator (Miller 2012:120-121). The game is successful in this regard because of a Giddensian reflexivity that relies on the players' preexisting knowledge of the rock musical and performance canon (Miller 2012:122). Indeed, part of any successful performance, whether of gender or a musical cover (which this game might be considered), is in the recognition of a calling-back-to the discursively familiar.

Miller's work, however instructive, is an anomaly in terms of academic research on rock music games. A significant amount of the literature on Guitar Hero and Rock 
Band consists of articles in game and music industry magazines as well as print and online reviews. Music industry magazines or entertainment/advertising publications describe the games' relationship to the music industry in terms of artists which lend their songs or likenesses to the game (Mettler 2008) or how popular they have become in people's households as a form of group entertainment (Bulik 2007, 2008; Croal 2008; Fritz 2007, 2008; Koerner 2008; to name a few). Reviews often pit the two games against each other in terms of what is offered to the player. Reviews (Bruno 2008, 2009; Crescente 2007) commonly fall on one side of the coin for different reasons: Guitar Hero is cited as being the first to offer the guitar-as-peripheral which is modeled closer to actual guitars. Rock Band, on the other hand, has a rapidly-growing song list and was able to get the rights to The Beatles' songs in order to create the critically-acclaimed Rock Band: The Beatles.

A recent edited volume on music games, Music Video Games: Performance, Politics, and Play is the closest source found looking at music games through more than their educational impact. However, the broader genre of music games is explored (including games such as Simon Says and the Mario Paint music composer) with a few discussing performance in games such as Guitar Hero, Rock Band, and Rocksmith. Mario A. Dozal (2016) explores notions of authenticity in Guitar Hero and Rock Band in order to explore in what ways creative authenticity applies to both music and video games, and what happens when these games opt for wider saturation in the market to the potential detriment of this creative authenticity, also known as “selling out." Daniel O’Meara (2016) takes a look at the tension between Rocksmith's role as a video game and role as 
guitar pedagogy tool, including the ways in which delivering an authentic guitar learning experience is potentially hampered by adherence to game design conventions.

Despite these recent additions to rock music video game literature, there is still a dearth in literature that takes a look at how these commitments to authenticity in music culture and game culture result in a particularly gendered virtual world with few if any opportunities for subversion outside of public play and more and more developers designing with intention of reaching a more diverse audience - a contribution that this study aims to make. 


\section{METHODOLOGY}

My methodological approach is designed to examine the relationships between rock music video game developers, the in-game content itself, and the players.

Developers for video game franchises such as Rocksmith and Rock Band are responsible for the design and implementation of in-game elements that, I hypothesize, are implicit in the construction of gendered discourses. The in-game content includes avatar creation and movements, as well as the songs available to play and loading screen text. The player's position can range in game and community involvement from casual at-home solitary play to small social group play and large-scale competitive play. To develop both a rich and provocative methodological approach, I draw from sources within the gender/virtual worlds literature which employ differing qualitative techniques.

I deployed content analysis of marketing materials, in-game text, and other visual aspects of the games themselves as part of my analysis. This follows a precedent set by previous research which employs content analysis to examine games and their marketing materials (Beasley and Standley 2002; Burgess, Burgess, and Stermer 2007; Dietz 1998; Downs and Smith 2009; Martins, Williams, Harrison, and Ratan 2009). The video games' covers, character wardrobe, and representation in industry magazines are examined for evidence of gender discrepancy. Through looking at these visual and textual representations of gender, the researchers conclude that women (when they exist in these virtual worlds) are constructed as passive, non-violent sexual objects of the male gaze. Even in situations in which female characters exhibit some form of agency (which can be few and far between), exaggerated feminine appearance is often more important than a realistic or natural depiction of women performing a variety of active tasks. 
Another subsection of the literature uses interviews and surveys often given after structured, experimental, and limited periods of play (Bartlett and Harris 2008; Beasley, Berrin and Standley 2002; Behm-Morawitz and Mastro 2009; Brown, Brown and Brown 1997; Dill and Thill 2007; Fox and Bailenson 2009; Gailey 1993; Hayes 2007; Nakamura and Wirman 2005; Norris 2004; Ogletree and Drake 2007; Winn and Heeter 2009; Yao, Mahood, and Linz 2010). Behm-Morawitz and Mastro (2009), for example, had male and female research participants play through two 15-minute sections of the adventure game Tomb Raider, which features protagonist Lara Croft in both a sexualized and nonsexualized position. According to the authors, 15 minutes of gameplay are enough to affect "self-esteem, self-efficacy, and gender beliefs after exposure to idealized images of the female body (815)." After their periods of gameplay, participants answered a survey, which found that playing the sexualized version of Lara Croft "resulted in less favorable attitudes toward women's cognitive capabilities" and "less favorable judgments about female physical capabilities (820).” Many of the aforementioned experimental studies come to similar conclusions, with players noting a decrease in self-efficacy or body image after gameplay with a sexualized avatar, showing a clear relationship between player and game. Ethnography and its online counterpart cyberethnography are another significant part of my research design and are unpacked ahead.

\section{On Cyber Ethnography}


Classic ethnographic research relies on participant-observation of a particular social group within a geographic area over a significantly long period of time.

Ethnographic research elevates observation and interviews with key informants and includes (especially where feminist ethnography is concerned) an emphasis on the reflexivity of the researcher and the voice of the research subject in order to provide a more complete—or Geertzian 'thick'—description of a culture (Berg 2009; Churton \& Brown 2010). Cyberethnography has significant similarities with classic ethnographic techniques, but takes place in online communities. While ethnomethodological introductory texts (referenced above) can refer to online forums or chat rooms as the primary home of cyberethnography (if it is mentioned as an option at all), games studies researchers have expanded the concept of cyberethnography to explore virtual and gaming worlds such as Uru, Second Life, and World of Warcraft, as well as "real-life" manifestations and extensions of virtual activities, such as competitive gaming tournaments and in-home console gaming (Pears \& Artemesia 2009; Brookey \& Cannon 2009; Taylor, Jensen, and de Castell 2009; etc.). "Real life" is presented in scare quotes so as to avoid what Nathan Jurgenson calls "digital dualism," the fallacy which states that online experiences are somehow separate from experiences in the physical world (Jurgenson 2011). The following examination of the ethnographic and cyberethnographic research informing my study will further elucidate the constant blurring between the avatar and the player, as well as the relationships between player, game, and creator. 


\section{Ethnographic Inspirations}

Helen Thornham's (2008) “It's a Boy Thing," uses an ethnographic approach to observe social play of console games in six gaming households. Interviews as well as observations of interactions between players in these households reveal insights about the relationships between players, gender, and preferences, namely that gendered preferences and attitudes about gaming are constantly in flux, performative, and context-dependent. Thornham specifically calls for studies that put the gaming environment in its proper context, rather than making assumptions based on mere content analysis or surveys of preferences and effects:

Separating gaming from where and with whom it occurs negates some vital and fundamental performative aspects of gaming which are conducive to the creation and support of pleasurable social gaming scenarios (138).

While Thornham outwardly states that she is not here to expand theoretical gender, she does make a nod towards the performative construction of gender by suggesting video games as a site within which gendered identities are produced (127). In particular, this concentration of social gaming speaks to my own research questions, which consider that there may be a difference in music game literal and gendered performance in different social scenarios (solo, small group home play, or public play in public spaces).

Taylor, Jensen, and de Castell (2009) look at the gender dynamics within the professional gaming industry by attending a series of competitive events and focusing not on the overwhelmingly male population, but on the roles of women that they did observe. Specifically, the authors examine what occurs when the lines blurred between 'work' and 
'play,' and what happens when gaming aims to become seen as a part of another masculinized entertainment—professional athletics. The authors find that there are limited avenues for women in competitive gaming scenarios, and they are often relegated to the role of cheerleader or groupie. When a woman decides to compete professionally, it is not completely unproblematic (the one female player in their study articulated her competitive prowess in one breath, but undermined the status of other women at the competition as only being there as groupies in the other). In a nod to theorist Judith Butler, Taylor, et. al. define gender as "contingent, embodied, and performative...always constructed (241)." However, they also bring in research on masculinities in order to unpack the myriad gendered, class, and racial privileges gained through the oppression of women.

\section{Cyberethnographic Inspirations}

While the aforementioned articles used a more traditional form of ethnography, the following cyberethnographies take the extra step of immersion into the virtual spaces they aim to research. A key element is an added level of participation through the creation of a character, avatar, or username in order to gain access to these worlds. The research reviewed in this section theorizes users' performances as they are embodied within these virtual spaces, which include online forums, games, and even virtual second lives.

Lori Kendall (2000) does not examine a game so much as the virtual textual communications of an online forum. While the forum, BlueSky, does have games available to play, Kendall examines its use as message board to express gendered identity 
performance. Kendall spent over two years as a participant on the BlueSky forums, devoting 11-20 hours a week online, and supplemented the observation with both formal and informal interviews as well as attendance at offline social gatherings. Kendall uses these online and offline interactions comparatively, as a means of gathering evidence of both online and "offline identity performance," acknowledging that the "real world" is just as performative as the virtual (258). I plan on making a similar acknowledgement in my own research, albeit through examining offline performance of a virtual performance.

Rather than turning to Butler to elucidate gender performance, Kendall uses preeminent masculinities research to shore up her argument that these text-based virtual spaces are where negotiations of hegemonic masculinity occur. Masculinities theorist Raewyn Connell (1995) defines hegemonic masculinity as "the configuration of gender practice which embodies the currently accepted answer to the problem of the legitimacy of patriarchy, which guarantees (or is taken to guarantee) the dominant position of men and the subordination of women (P. 77).” The predominantly white male middle-class user base of this BlueSky forum both performs hegemonic masculinity while simultaneously explicitly rejecting it. Online forums are seen as a means by which racial and sexual minorities can hide behind anonymity and textual (rather than visual) representations of self, but they ultimately require users to perform hegemonic male whiteness in order to gain the acceptance of the dominant group. This research is a constant reminder not to focus on just the performances of femininity in my subject of study, but to examine performances of hegemonic masculinities as well.

Klasptrup and Tosca (2009) conduct ethnography within the massively multiplayer online game World of Warcraft (WoW)'s realm of Azeroth to shed light on 
the importance of clothing and fashion in virtual worlds. The authors mostly draw on cultural research that emphasizes the importance of fashion as a means of expression and symbolic communication, and they theorize that such an importance exists in the virtual world. Klasptrup and Tosca, both WoW players, conducted participant-observation within the world and used their observations to conduct surveys and structured interviews. They found that players did, in fact, see in-game clothing options as more than a means of obtaining armor or skill-boosting properties - they saw it as fashion, which explains why players would sometimes opt to wear inferior gear. Gender does not figure as significantly within their study as fashion does, but their research does show an increased use of fashion for male players as well as female players (who are stereotypically the purveyors of fashion).

Domna Banakou (2010) looks at the effects of avatar appearance on social interaction in the virtual world of Second Life. Second Life is an online virtual world in which a user creates an avatar and interacts with other residents in a 3D environment. Banakou gave research participants two avatars which matched their "real life" gender identity, but with significant differences: one of the avatars was the standard, basic avatar one starts with in Second Life, and the other avatar has a more elaborate (and conventionally attractive) "skin," with more detailed hair and body shape. Research participants were given a slightly different research task for them not to influence the actual research outcomes, and their communication with other users was observed by a researcher with their own avatar in-world (8). Banakou found that female avatars in general have a more successful time communicating with others in online encounters, and the more conventionally attractive (and one would argue, more sexualized, gender 
stereotypical) avatar was more successful in both initiating and continuing social encounters. The study confirms previous research that states that "real life" stereotypes and norms often reflect themselves in the virtual, a claim that I also aim to examine in music game virtual worlds.

Dumitrica and Gaden (2009) also examine gender differences in Second Life, but do so in a way that drives both ethnography and virtual worlds research forward. The authors use collaborative autoethnography, examining the ways in which they perceive the performance of gender in the Second Life environment. They engaged with Second Life for six months, keeping a field journal and constantly revisiting and discussing their experiences with one another. Dumitrica and Gaden hold to a post-structuralist feminist perspective, drawing from theorists Judith Butler and Donna Haraway. The authors understand gender "as being performed through our actions, behaviors, and choices; we do gender (5)." An important part of their use of autoethnography is acknowledging Donna Haraway’s (1988) feminist epistemological perspective called situated knowledges, or situated perspectives. Acknowledging how one's own perspective influences researcher experiences is a key part of successful situated autoethnography. Far too often, the researcher's experience is often a removed to create a God's-eye objectivity in virtual worlds research (even those that involve ethnography). Dumitrica and Gaden utilize autoethnography to connect their interest in gender perspectives in Second Life with their own situated perspective. Autoethnography serves to connect the researcher, the world being examined, and the wider networks of power within which these virtual worlds work (7). 
Dumitrica and Gaden find that, despite the supposed liberatory nature of Second Life, gender discourses are alive and well from the very moment of avatar creation, where the gender binary is inescapable (even for non-humanoid avatars). Gender continues to present itself through the commerce of sexualized skins and "pose-balls" that animate different avatars in gendered ways. However, they found that increased exposure to the environment eventually led to normalized gender scripts, to the point of taking the gender dynamic for granted. Thus, the authors conclude that gender performance is complex, particularly when taking their own positions combined with "the social vision (re)created through the social/technical infrastructure (18)." Gender representations and opportunities for gender performance in virtual spaces can be both liberatory and limiting, and contextually complex, particularly when public corporeal performance can be witnessed alongside that of the performing avatar in the Guitar Hero/Rock Band case.

Celia Pearce and Artemesia's Communities of Play (2009) looks at the development of an online community and what happens when the virtual world in which the community is based is shut down. Pearce chronicles the ensuing migration and creation of a diaspora through in-depth cyberethnography over the course of eighteen months, including the chronicling of "real life" meetings between community members. In her study, she performs feminist ethnography, specifically, by making sure to retain as much of her participants' voice as possible, even having them comment on chapters throughout the book relatively unedited. Cyberethnography, for Pearce, is a particular useful method for video games, and is further complicated by the nature of the technology: 
Game ethnography is one of the rare circumstances in which an ethnographer is required, to varying degrees, to actively participate in the culture she is studying, not to simply observe it. The reason for this is more technical than philosophical: you cannot observe a virtual world without being inside it, and in order to be inside it, you should be "embodied (196)."

Autoethnography figures so prominently in Pearce's work, she devotes an entire chapter to her experiences as an avatar and even names her avatar as co-author. In this chapter, she expands the autoethnography beyond the virtual space and examines the process of presenting her findings at conferences, where she experiments with presenting in her narrative voice as well as the perspective of her avatar, Artemesia. This brings up an interesting dynamic for this study, where I created my own avatar (becoming embodied in game) and customized it to play through the Rock Band video game. For Rocksmith, which did not include avatars, my physical form became the vessel for rock guitar prowess. My own autoethnography in this work, in part, navigates a similar experience as a virtual avatar and as a performer.

\section{The Creator-Game-Player Dynamic}

The dissertation examines the dynamic relationship between game creators, the game itself, and the players who consume it. In cyberethnographic research, the CreatorGame-Player dynamic expresses itself in similar ways. The games become the impetus for forming communities of players, whether as leisure activity or competitive vocation. The virtual worlds in the ethnographies are more likely to take the role of set dressing for 
social interactions than being the subject of analysis itself, something which I hope to better flesh out in my own research.

The players, who consume these virtual worlds, and their subject positions, are considered in different degrees of depth. For Klastrup and Tosca (2009), demographics are given a cursory nod, but the player relationship with clothing is more significant. It would be interesting to see how a player's in-game relationship with clothing options and fashionable expression compares to their "real world" expression: In both "real" and virtual life, a person's race and class would figure heavily into the analysis, as the person/avatar with the most economic and cultural capital_ "real life" money, Azerothian gold and experience/levels - would have the most access to "fashionable" clothing. Lori Kendall (2000), on the other hand, acknowledges the importance of her subject group's position as predominantly white and middle class in shaping performances of masculinities within a virtual space. On the other end of the spectrum, Dumitrica and Gaden (2009) hold player subject-position as paramount to their study, as they occupy the position of players themselves. The authors' positions as female, white, middle-class, and yet new to the technology they were studying all had an effect on how they initially saw the world, as well as how their interactions changed over time. Celia Pearce represents perhaps the most complete examination of player subject-position, as she combines her own subject-position, her analysis of the users' subject-position, and having the users speak for themselves. In this way, one can see the complex network of relationships between perceptions of social performance.

The most notable similarity between the ethnographies examined here is how little the creators figure into the analysis. While Pearce does explore developer 
motivations and responses to the migration between communities in her long-form ethnography, there is little to no discussion of creator motivation in the creation of game elements themselves. The same can be said for the other ethnographies discussed here, which take into account the players'/users' relationships with each other and the game, rather than the community of individuals that are responsible for the construction of the virtual worlds they inhabit. Because so much of what the researchers see as gendered interaction is due to designed elements, knowing the particular subject-positions of the designers is necessary to provide a more complete picture.

\section{Methodological Approach}

My methodological approach consists of a mix of quantitative analysis, discourse analysis, semi-structured interviews, surveys, and (cyber)ethnography to examine the Rock Band and Rocksmith franchises. This approach provides a description of the dynamic between creators, the games, and the players through inclusion of (cyber)ethnography at four basic points: the creators, the games, the players, and autoethnography. Ahead is an outline of the precise methods and techniques used, followed by more explanation of each point of intersection. 


\begin{tabular}{|c|c|c|}
\hline Point of Analysis & Technique & $\begin{array}{c}\text { Sample Size/Number of } \\
\text { Observations/Details }\end{array}$ \\
\hline $\begin{array}{c}\text { The Creators: Ubisoft and } \\
\text { Harmonix }\end{array}$ & Semi-structured interviews & $\begin{array}{l}3 \text { Ubisoft interviews, } 4 \\
\text { Harmonix interviews, } \\
\text { coded in MAXQDA }\end{array}$ \\
\hline Rock Band: The Game & Playthrough & $\begin{array}{l}5 \text { hours of play, } 10 \\
\text { screenshots }\end{array}$ \\
\hline Rock Band: The Text & Text/content analysis & $\begin{array}{l}60 \text { pages of text, coded in } \\
\text { MAXQDA }\end{array}$ \\
\hline Rock Band: The Song List & Statistical analysis & $\begin{array}{l}2,000 \text { songs, analyzed in } \\
\text { STATA and Excel }\end{array}$ \\
\hline Rocksmith: The Game & Playthrough & $\begin{array}{c}\text { Over } 200 \text { hours invested } \\
\text { (2011 and } 2014 \text { versions), } \\
12 \text { screenshots }\end{array}$ \\
\hline Rocksmith: The Song List & Statistical analysis & $\begin{array}{c}1,000 \text { songs, analyzed in } \\
\text { Excel }\end{array}$ \\
\hline $\begin{array}{c}\text { The Players: Avatar } \\
\text { Identity }\end{array}$ & Informal interviews & $\begin{array}{c}8 \text { interviews conducted via } \\
\text { Skype, } \\
\text { snowball/convenience } \\
\text { sampling }\end{array}$ \\
\hline $\begin{array}{l}\text { The Players: Music Games } \\
\text { and Gender }\end{array}$ & Survey & $\begin{array}{c}\text { Snowball/convenience } \\
\text { sample, } 163 \text { participants }\end{array}$ \\
\hline The Players: Public Play & Participant observation & $\begin{array}{l}4 \text { locations (two Rocksmith } \\
\text { events, two Rock Band } \\
\text { events), } 39 \text { argete. } 30 \text { hours } \\
\text { invested }\end{array}$ \\
\hline $\begin{array}{c}\text { The Players: Gendered } \\
\text { Experience }\end{array}$ & Semi-structured interviews & $\begin{array}{c}\text { One Rock Band night } \\
\text { participant, one } \\
\text { professional player, coded } \\
\text { in MAXQDA and by hand }\end{array}$ \\
\hline
\end{tabular}

Table 1. Methods and research techniques.

The creators 
Participant observation was conducted at the Harmonix offices in Cambridge, MA, and the Ubisoft offices in San Francisco, CA, where these observations gleaned an understanding of office culture, demographics, and power hierarchies that contribute to game design decisions. Semi-structured interviews were conducted with three members of the Rocksmith team and four members of the Rock Band team at or near the offices, then continued via e-mail. Personal communications over four years of working at and attending industry events also contribute to the picture of these games' development intents.

The interviews often coincided with major industry events that I was also attending (the Game Developers Conference in San Francisco, CA and the Penny Arcade Expo East in Boston, MA). Lining interviews up with these events posed both advantages and limitations. The advantage was that I could travel once to these locations for participant observation at the conventions, interviews, and office visits rather than splitting these activities up into increasingly cost-prohibitive trips. The disadvantage of lining research with the creators with major industry events is scheduling enough time for a rich interview around their attendance at these events. During the Penny Arcade Expo East in Boston, for example, the Harmonix team had to prepare for several panels, and their CEO Alex Rigolopolous was preparing for the keynote address. In addition, the day of the interview, the office had also scheduled a visit from a group of fans to playtest their newest announced game, Chroma. The team was also in the process of working on several games, announced and unannounced, in the process of the office visit, which led to certain rooms either being off-limits or having to be cleared of any content.

The game 
The franchises being researched here do not share the exploratory, open worlds of other virtual spaces. The predominant goal of these games is to successfully perform songs whether solo or in small cooperative groups, and there is sometimes a narrative thread that strings these performances together into a story mode. There is, however, an avenue in which avatar interaction can be used for cyberethnography. Although interactions between avatars are designed into the game, one can argue that, through playing through the story mode in Rock Band, for example, ethnography can be conducted: one creates a band at the beginning of a playthrough, follows them through cutscenes and stage performances, observes their (designed) behaviors, and draws conclusions about Rock Band "band" culture (as well as gaming and music culture overall) by following one virtual band's journey.

I created an avatar in Rock Band and played through the on-disc content for approximately five hours. I captured video from my playthroughs through Blackmagic capture hardware. The intention was to play through the entire game, but, due to the size of the files and inability for the capture hardware to record more than one song at a time, the playthroughs were limited in length. However, in conjunction with developer interviews on avatar animation and text analysis, I believe that these limited playthroughs reached enough saturation for analysis of movement and player interaction.

Harmonix provided a document with every piece of in-game text from the original Rock Band for analysis. After cleaning and organizing the text, which included the assets' file names, I was left with approximately 60 pages of text in the form of loading screen tips and descriptions for all of the items (clothing, accessories, make-up) one can 
use to customize their character. I coded the text in MaxQDA, with different code groups for loading screen text and clothing/accessories.

\section{The players}

Unlike the ethnographies explored here, there is no massively multiplayer online component to the games being studied. In the Rock Band franchise, only four avatars at a time are on stage, typically played by up to four people in the same room (there was no online multiplayer in Rock Band until the introduction of the feature in Rock Band 4 Rivals expansion, released in 2016). Because of this, I limited my ethnography to the following player experiences: the play session that occurred in my living room, a public performance and subsequent interview at a local Rock Band night at bars, and two casual performances at regional/national-level conventions. In addition, I conducted a survey of players that focused on their attitudes towards rock music video games and gender's place in them, as well as an interview with a woman who played Guitar Hero at the professional level. The restriction of these games to more closed ecosystems rather than massively multiplayer virtual worlds, I hope to construct a more complete ethnography using the various ways music gameplay presents itself-in itself an innovative approach, as it is a little-researched genre - as well as a general look at how console gameplay extends beyond the living room and into public spaces, and how gender discourses and performances shift within these different contexts. 


\section{Autoethnography}

In autoethnography, "the researcher does not enter and study people in a new milieu from the outside - the researcher is the insider (Dumitrica and Gaden 2009:8)." When it comes to this research milieu, I am in various ways an insider: a musician with intimate knowledge of the music industry and performance, a self-professed gamer, and a gaming industry insider, having personal experience working and interacting with the companies I aim to research. As such, my experience with the games and with the industry cannot be removed from the research. Much like Pearce (2009), I aim to make autoethnography a significant part of the dissertation research, for, as Dumitrica and Gaden (2009) assert:

...by allowing researchers to compare their own paths through the Internet, as well as the paths through which they reach certain conclusions about online worlds, ... autoethnographies provide 'thick' descriptions (Geertz 1973) which incorporate not only the context of experiences, but also the feelings and expectations associated with them (9). 


\section{THE “ROCK DOC:” AN AUTOETHNOGRAPHY}

\section{Prologue: Putting the Rock in RockDoc}

An early picture of me, a few days after I was born, shows my first balled up, mid-yawn. My family performed a little revisionist history to say that this picture shows that I was always meant to be a singer. I received my first guitar when I was a toddler. It was plastic, and it had buttons that wailed with a different guitar riff when pressed. You could attach a microphone to it and hang it around your neck. I remember writing songs with it—about Mother's Day, in the style of the Beach Boys; about a spirited event at my mother's advertising agency where people wore ridiculous hats (called, simply, "Hat Day"); about state capitals when I was trying to learn them. I retained nothing except the drive to do more. Fast-forwarding to my high school years, I took all of that typical teenage angst and channeled it into song with the aid of my then-boyfriend on guitar, first as an acoustic duo, then as a full band (but still billed as a solo artist), then as pop-metal band Crimson.

In my formative years, I was exposed to the 80 s hair metal that my brother enjoyed, and the androgynous looks that accompanied them. I loved that these were fast songs, had musical elements that pleased me, crunching guitars, but their voices were high enough that I could conceivably sing along. And they wore makeup! In the mid-90s, I found myself inspired by artists such as Selena, whose voice had a distinct rock growl to it despite having Tejano and banda roots. She married her guitar player. When No Doubt's Tragic Kingdom burst onto the scene, I had another model: a singer who channeled very personal pain into clever lyrics and high-energy instrumentation. She 
dated her bassist. My discovery of Pat Benatar was part of a nostalgic rabbit hole that I went down in the search for songs to sing in the same vein. She married her guitar player. The women in my life were singers, always singers. And bass players? My most prominent memory was Tia Carrere's Cassandra and her slithering frame (and rhythm — it was all over the place) in Wayne's World. But she was a singer still. Guitar players were to be admired and married, but never to become.

In the process of becoming a Puerto Rican Gwen Stefani/Pat Benatar hybrid, I ran up against several roadblocks that would eventually be echoed by other women in the industry, in the pages of the feminist musicology articles I'd read. In one meeting with an entertainment lawyer (who'd agreed to shop my music around to record labels), I asked him when we could come back and see him. He said, "If you're wearing those boots? Anytime." After over 15 years of calling myself a singer in earnest, I find it hard to wade through the gigs and rehearsals and booking calls and load-ins to pick out more individual instances such as this. It just feels like a general malaise. I never feel like there is anything extremely damning, but more like little papercuts: my now-husband telling me not to wear my engagement ring on stage so I could seem more available; a kaleidoscope of honeys and sweethearts and catcalls. You learn the subtle difference between a cheer for the sway of the hips versus the one that comes after you hold out a note slightly longer and higher than people would expect. You learn you get more of the former. You walk into a guitar store, and you can fully expect to never be addressed, even if you're playing, even if you're buying. The spotlight can feel like a target, making it even more important to check off the list of steps to protect yourself that rape culture 
dictates: watch your drink, don't accept drinks from others, hold your keys between your fingers in a fist so you look like a janitorial Wolverine as you walk to your car.

I found my performances onstage getting more aggressive. I headbanged more, I kicked more, and I would contort my face to match the intensity of whatever growl I was emitting. It was my own version of what Karen Eileraas calls "performing ugliness as resistance (1997: 3)" where ugly voices, screaming, and dress and performance can be employed as defiance against a society that aims to control the volume and quality of a woman's voice.

As both "Elisa Meléndez" and Crimson, I had the opportunity to be a part of a local woman-centric music festival, Femmefest. Initially, I faced an interesting internal struggle: did I want to be a part of this festival and further pigeon-hole myself as a "female-fronted rock band" to the detriment of my being taken seriously as just a "rock band?" Or was I so wrapped up in playing with the boys (literally and figuratively) that I would miss out on having a sense of community with my musician sisters? These experiences stoked fires of frustration and the drive to make things better, and it was the Women's Studies program at FIU that gave it all a name.

\section{Introducing Eventides}

As I pursued further research into feminist musicology, I continued to connect and reconnect with another love of mine: video games. Video games provided a family bonding experience when I was a child, my earliest memories consisting of watching in awe as my father made his way through Tetris boards, my mother matching colors in Dr. Mario, and being my older brother's co-pilot and reading him Nintendo Power magazine 
tutorials on how to kill difficult bosses as he hacked and slashed his way across fantasy worlds. I continued to find games a source of entertainment and inspiration, moving from the late 80s Nintendo Entertainment System to PC and an Xbox console. When I found World of Warcraft, it was one of my first experiences taking my family and solitary activity and making it more public, playing with other people around the world in a massively multiplayer online role-playing game (MMORPG).

When it came time to create a character, I chose to create a female character. I so rarely had the opportunity, as the most common woman that existed in video games at the time was the absence of one (Dietz 1998). I created a female Draenei, a sci-fi centauresque creature with violet skin and horns, and I named her Eventides. "Eventide" was a nickname my husband bestowed after my love for harmonizing with everything when I sang_Eventides being a brand of harmonizing hardware. It became pluralized when "Eventide" was already taken on the server I was playing on. It was a name that would follow me around several games and online presences, and it became useful as an alternative to my other online persona, "ElisaRockDoc," when I wanted to remain hidden. I would soon find, however, that presenting as a woman in the video game space, whether it was as a Draenei, a cartoonish version of myself as avatar, or as an "internet personality" in my own right, came with its own set of challenges with few avenues for escape.

\section{Eventides in the Spotlight}

I play the majority of video games on the Xbox console. The console not only allows the ability to play games of multiple genres, but it can allow you to both play and 
communicate with other people through an internet connection. A community of online players is built through the Xbox Live service, which provides a number of ways to find and interact with other members, including the Xbox Live Community Spotlight on the Xbox console dashboard. This is a weekly segment in which a member is chosen and their profile is displayed for a week on the community website as well as on the community section on Xbox consoles. Community members are often nominated by friends via e-mails to the Xbox Live community director. If selected, a member fills out a brief questionnaire asking about general likes and dislikes from gaming and popular culture. The profile, along with some of the questionnaire answers, is posted up for a week and then archived.

I received such a nomination in October 2011 from the Xbox Live community director. I filled out the questionnaire, which asked me such things as my favorite comic book, and to describe the worst job I ever had. A couple of weeks after sending back the questionnaire, my profile was listed on the Xbox community's spotlight. For reference, Figure 1 shows how I styled my avatar on the Xbox Live platform. If you visited the website or saw the dashboard, you would also see my avatar in motion, where it would summon a black housecat and interact with it: 


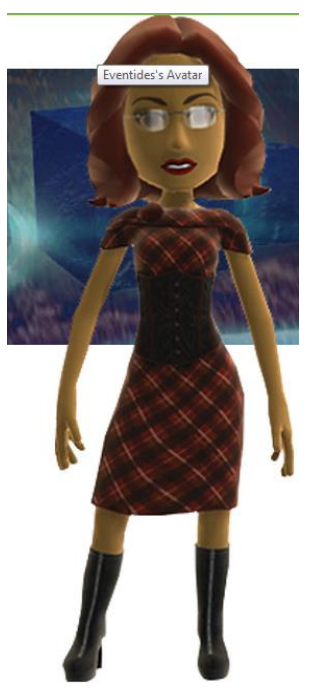

Figure 1: Eventides the Avatar on Xbox Live. Retrieved from https://account.xbox.com/en-US/CustomizeProfile in October 2011.

I found that, on the first day of my Xbox spotlight appearance, I had received 100 messages in my Xbox Live inbox (the maximum allowed at one time). Interested in the kinds of responses I would receive, I documented any unique message that wasn't just a salutation (as the Xbox spotlight concludes with "Say hi!" and many users followed suit saying simply that). Over the course of a week, I received 1,091 of these unique nonsalutation messages. After performing some rudimentary coding, I was able to produce ten categories of messages I received:

Congrats \& Spotlight Acknowledgement (18\%): These were messages that either congratulated me for being on the gamer spotlight ("Congrats!"), or somehow acknowledged that I was on it ("Hey, saw you in the spotlight!").

Come-Ons (8.2\%) and Denigration (8.7\%): Together making up 16.9\% of the messages, these are the two categories that most interested me, as they were the responses I most 
expected as a result of presenting myself as a woman online. The Come-Ons were attempts at flirtation or seduction, while I categorized denigration as outright insults and abuse, including two pictures of genitalia. The insults were often gender-based ('bitch', 'ho,' 'slut,' etc.) or sexuality-based ('dike [sic],' 'fag,' etc.). More discussion of this particular set of categories follows.

Music (15.1\%): As part of my interview and my Xbox Live profile, I made mention that I was a lead singer in a hard rock band, Crimson. I also included a link to the band's website. These were messages that acknowledged my band in any way, from questions about where to find our music to critiques. Surprisingly (and I say 'surprisingly' because of the above category), the responses in this category were mostly positive.

Friend Requests (15.1\%): Simply, these were people that wanted me to add them to my friend's list. Because the messages came fast and loose over the spotlight week, my inbox was often too full of requests for people to send their own. That was a common complaint in this category.

Gaming-Related (11.4\%): This category consisted of miscellaneous messages where people asked to play specific games with me, asked me questions about Xbox Live, or asked me to provide them with codes or help (possibly thinking that I was a developer or worked in the industry somehow). 
Kindness (9.1\%): These messages included general niceties such as "You rock" or "You sound cool."

Academic (6.3\%): These were questions or comments having to do with my field of research. On my profile, I listed that I was a Ph.D. student studying gender and video games. One voice message I received assumed my "rock doc" nickname meant that I was a geologist and asked why I would study something like that.

Me/Biographical Questions (4.7\%): These were general biographical questions, asking me how old I was or where I was from.

Misc. (4.6\%): These messages were miscellaneous messages that did not fall into the above categories and were often odd, nonsensical, or literal gibberish. The first category can be chalked up to simple recognizance. At the time, the spotlight was a relatively prestigious category, with selection and inclusion not being the most transparent process (my inclusion was as a result of being someone the person in charge found interesting and a potential role model).

The second set of categories was the least surprising and the most relevant to my current research. As a result of my presenting as female online, I fully expected my entrance in a typically male-dominated space to be met with either flirtation or harassment/rejection. Academic Mia Consalvo notes that, when she first began her research into games and player culture in the early 2000s, "it was considered news by the 
mainstream press that women played videogames (2012: 2)." Now that women are playing en masse and are more visible, she states:

The rage we see expressed by threatened individuals and groups seems to be based on at least two factors - sexist (as well as racist, homophobic, and ageist) beliefs about the abilities and proper place of female players, and fears about the changing nature of the game industry (2012: 3).

This reaction manifested itself in both the Come-Ons and Denigration categories, which I further outline below.

\section{Doing dishes and blow jobs}

In games where I have the option of customization, I often opt to make the avatar resemble me as much as possible, which usually means a female avatar with a mix of feminine and casual clothing/make-up expression. By presenting as a woman online, I left myself open to an unequal amount of targeted gender-based abuse which plagues the gaming industry. The second greatest category I received ranged from what could be considered mild flirtation to outright hostility. Some users opted for using tropes related to depictions of femininity circa the American mid-20 $0^{\text {th }}$ century, such as housework and cooking (see Figures 2 and 3): 


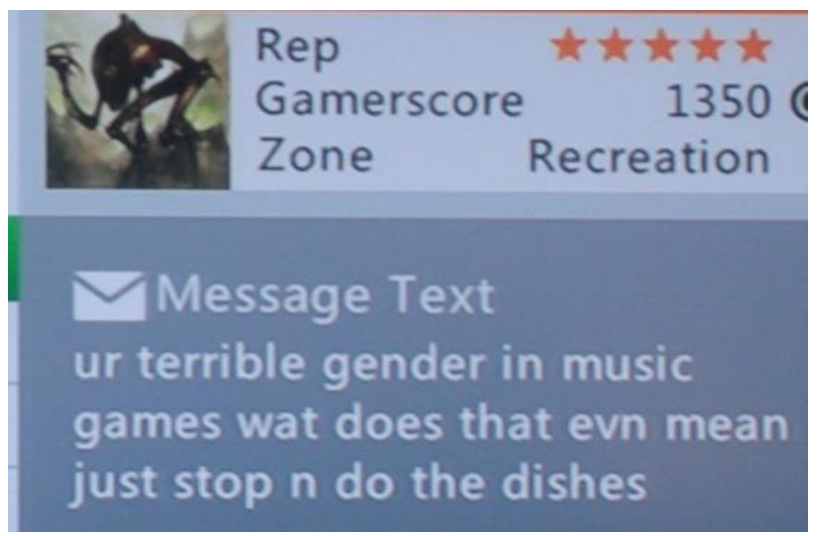

Figure 2: Sending Eventides to the kitchen. Retrieved from Xbox Live in October 2011.

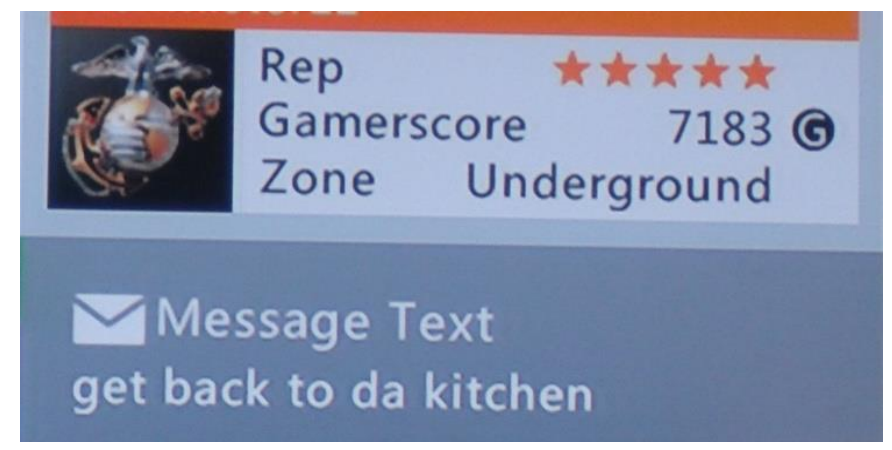

Figure 3: Sending Eventides to the kitchen. Retrieved from Xbox Live in October 2011.

Other users requested that I send them pictures, chat with them using the video chat feature, or directly requested that I show them my breasts, as seen in Figure 4:

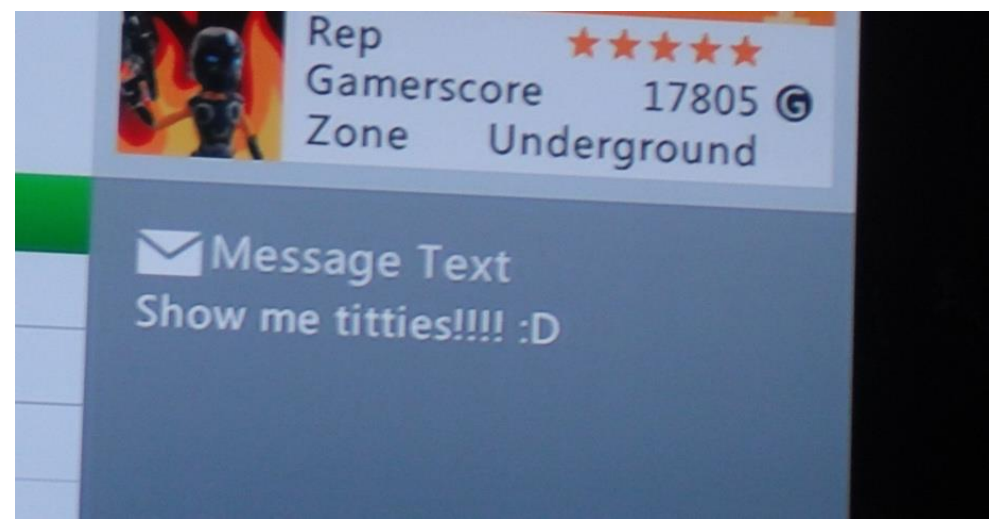

Figure 4: Asking for it. Retrieved from Xbox Live in October 2011. 
More graphic messages included pictures of male genitalia with requests to touch them and graphic depictions of sexual violence and physical abuse (see Figures 5 and 6):

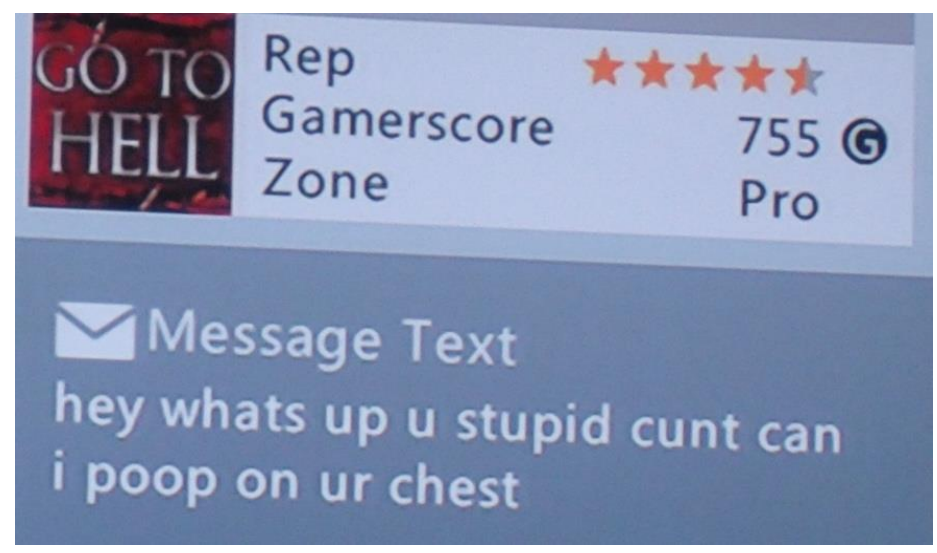

Figure 5: A delightful greeting. Retrieved from Xbox Live in October 2011.

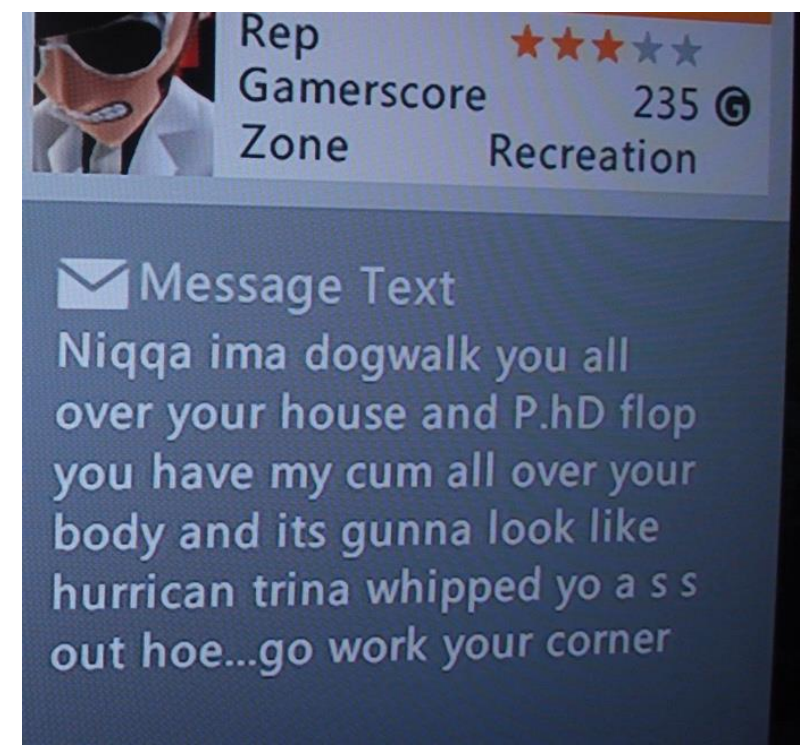

Figure 6: Really painting a picture. Retrieved from Xbox Live in October 2011.

These insults, many of which are gender/sexuality-dependent, are based on the assumption that the gender identities of the avatar and its wielder are matched. If the 
avatar is female, you must also be a woman. The "natural attitude...that cements the link between genitals and gender" is carried into the virtual world where the link between avatar gender and real-life gender is cemented (Currah \& Moore 2004). As later chapters will show, this experience is not unique to me. In fact, colleague and FIU Ph.D. holder Connie Viamonte carried the weight of this shared experience in her own work, where she wrestled with character creation and the consequences of her avatar choice in World of Warcraft (Viamonte 2015). I am thankful for Viamonte, other games studies researchers, as well as this very Ph.D. program for helping me build some sort of critical distance between me and my work, albeit a small one, that allowed me to endure and catalog these messages in the name of research instead of allowing these micro- and straight up aggressions to dampen my spirit of play, as it does for so many others.

\section{Eventides, the Frag Doll Cadette}

One reason I didn't feel disempowered by my Xbox Live experience was because of the community I was part of at the time: the Frag Dolls. As I started my Ph.D. work, someone suggested that I look up the Frag Dolls, a cadre of professional female gamers hired by video game publisher Ubisoft in order to compete in and help market their games. As luck would have it, this research occurred at the same time that they opened up their Frag Doll Cadette program, billed as an internship that could aid women interested in getting their foot in the door of the gaming industry. The internship consisted of content creation (videos, livestreams, and blogs) as well as supporting Ubisoft products and helping market them at gaming tradeshows such as the Penny Arcade Expo and San Diego Comic-Con. The program straddled the line between genuine desire to change the 
industry for the better and capitalizing on the relative novelty of having a gaggle of women at a booth. I applied and was accepted for their Fall 2010 class, and I continued to work with them on a part-time basis until 2014.

My first event was at the Penny Arcade Expo in early September of 2010. I was tasked with helping to show off Just Dance 2, a dancing game, and HAWX, a flight simulation game. As a significant portion of the Cadette work would take place at events, one of the first conversations I had with the planning team was about our uniform at the event, our comportment while at the booth, and about the elephant in the room: "booth babes." Booth babes are the slang term for promotional models which were typical of trade shows (especially trade shows in male-dominated fields) that are hired to attract people to the booth and help inform attendees about products. Complaints about the use of such models are many and varied. Women (and they are primarily women) are being objectified for the use of selling product. Women are often scantily clad, leading to the conclusion that heterosexual men are the intended audience at these events, shutting women out of the conversation (which falls into the heteronormative trap of thinking that men are the only ones that would enjoy attractive women). Promotional models are often hired purely for their looks versus their product knowledge. That reputation leads to women developers not being taken as seriously as their male counterparts when they staff their own booths. I have heard countless stories of women developers being asked if "I could speak to someone who actually worked on the game," not realizing that they were standing in front of someone crucial to development.

The Frag Doll Cadette program attempted to combat the booth babe stigma while still reaping the benefits from having women at a booth at what was still a male- 
dominated trade show. After my first show, I thought about a typology (see Table 2) that differentiated the "Frag Doll Cadette" from the "booth babe" based on guidelines we were given and observations at the convention:

\begin{tabular}{|c|c|}
\hline Frag Dolls \& Cadettes & Booth Babes \\
\hline $\begin{array}{c}\text { "Gamer uniform:” jeans and t-shirts } \\
\text { Player agency; could actively play games } \\
\text { for attendees }\end{array}$ & Tight clothes, dresses/skirts, high heels \\
\hline $\begin{array}{c}\text { Assumed game knowledge } \\
\text { Make-up recommended }\end{array}$ & Assumed game ignorance \\
\hline $\begin{array}{c}\text { attendees } \\
\text { Internship program, ongoing relationship } \\
\text { with company }\end{array}$ & Make-up required \\
\hline
\end{tabular}

Table 2. Typology of Frag Dolls and Booth Babes, as interpreted through Frag Doll guidelines.

Despite the attempts to combat the "booth babe" stigma through deliberate uniform choices, being imbued with the agency to play the games we were showcasing, and the promotion of our cadre as a kind of internship program with the intent of positive social change, we were not able to escape it. In fact, after my very first show with them, I found several pictures of Frag Dolls and Cadettes featured in a website slideshow on prominent video game press outlet G4TV for "Cosplay and Booth Babes.” As we were not wearing costumes related to video game characters, one assumes that we fell into the latter category. For comparison, other entries in the gallery included women promoting Gamecrush.com, a now-defunct website where people could pay money to chat and play 
games with a woman (see Figure 7). They were depicted wearing t-shirts with bullseyes on the chest, reading "Play Me."

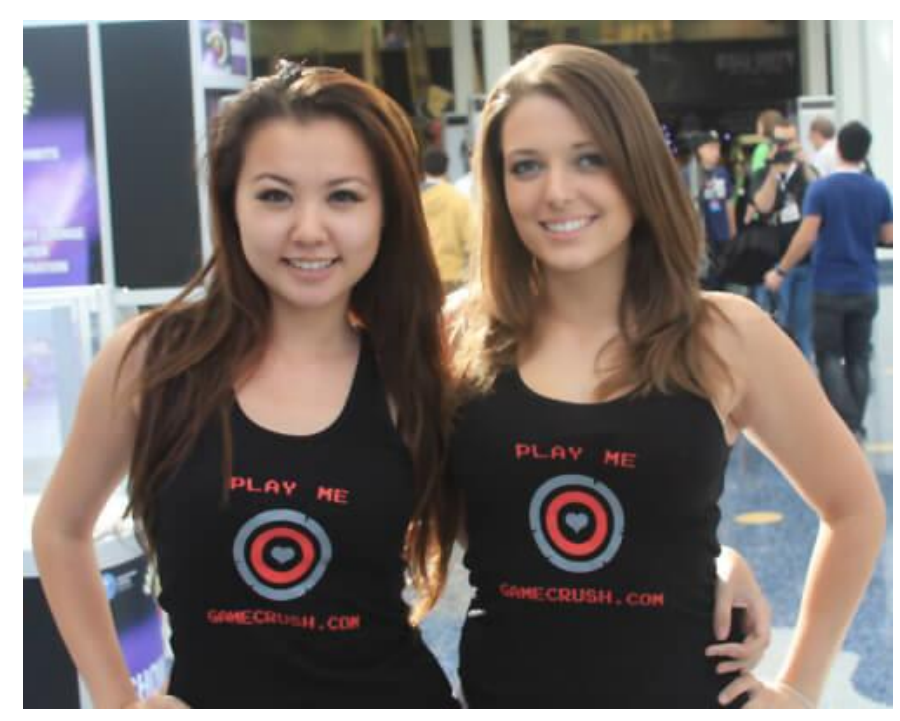

Figure 7: GameCrush.com "booth babes" at PAX East 2010. Retrieved from https://cdn.gamerant.com/wp-content/uploads/GameCrush-girls-1.jpg May 1st, 2017.

Both the GameCrush service and the models that helped promote it were emblematic of the industry: women were/are in such scarcity that you had to pay in order to experience one, leading to potential suspicion when women appear en masse. The term "booth babes" implies sexual attractiveness and a location-based presence- they're there for the booth work, and nothing more. The image was hard to shake throughout my tenure as a Frag Doll Cadette, with people at events distrusting our ability to play the games we were showcasing, despite our attempts to make clear our skill through performative play, literally showing ourselves playing the game frequently during livestreams and at events (and even then, there were accusations of us miming our hand movements on the controller to a pre-recorded video of gameplay). 
More despite it than because of it, being a Frag Doll Cadette did provide a lot of members the opportunity to make connections with industry professionals in an effort to get their feet in the door. Several Frag Dolls and Cadettes went on to become producers, community managers, event producers, and esports hosts at major studios. Since my first event experience, in turn, conventions like PAX have instituted a ban on "booth babes," citing a commitment to not alienate multiple growing segments of the player population. While explicit "booth babe" hiring is dwindling, some companies are circumventing this by having cosplayers (people that dress up and act as a fictional character) at their booths. Because female characters are scarce and often sexualized when they do exist (Dietz 1998), the "attractive woman calling siren-like to male nerds" marketing tactic is maintained and more insidious when couched as a commitment to game accuracyproving that women's virtual and corporeal representation is still an uphill battle.

\section{"ElisaRockDoc is Now LIVE!"}

One increasingly popular form of consuming content about video games (in addition to reading blogs and news sites and watching online videos) is watching live broadcasts of gameplay via streaming sites such as Twitch, YouTube, and Mixer. Typical broadcasts involve sharing the gameplay view from the player's console or computer and having a camera pointed on them to showcase both gameplay and reactions in real-time, as well as being able to carry on live conversations with the audience on these sites. Figure 8 shows a typical view on the Twitch.tv website during one of my own broadcasts: 


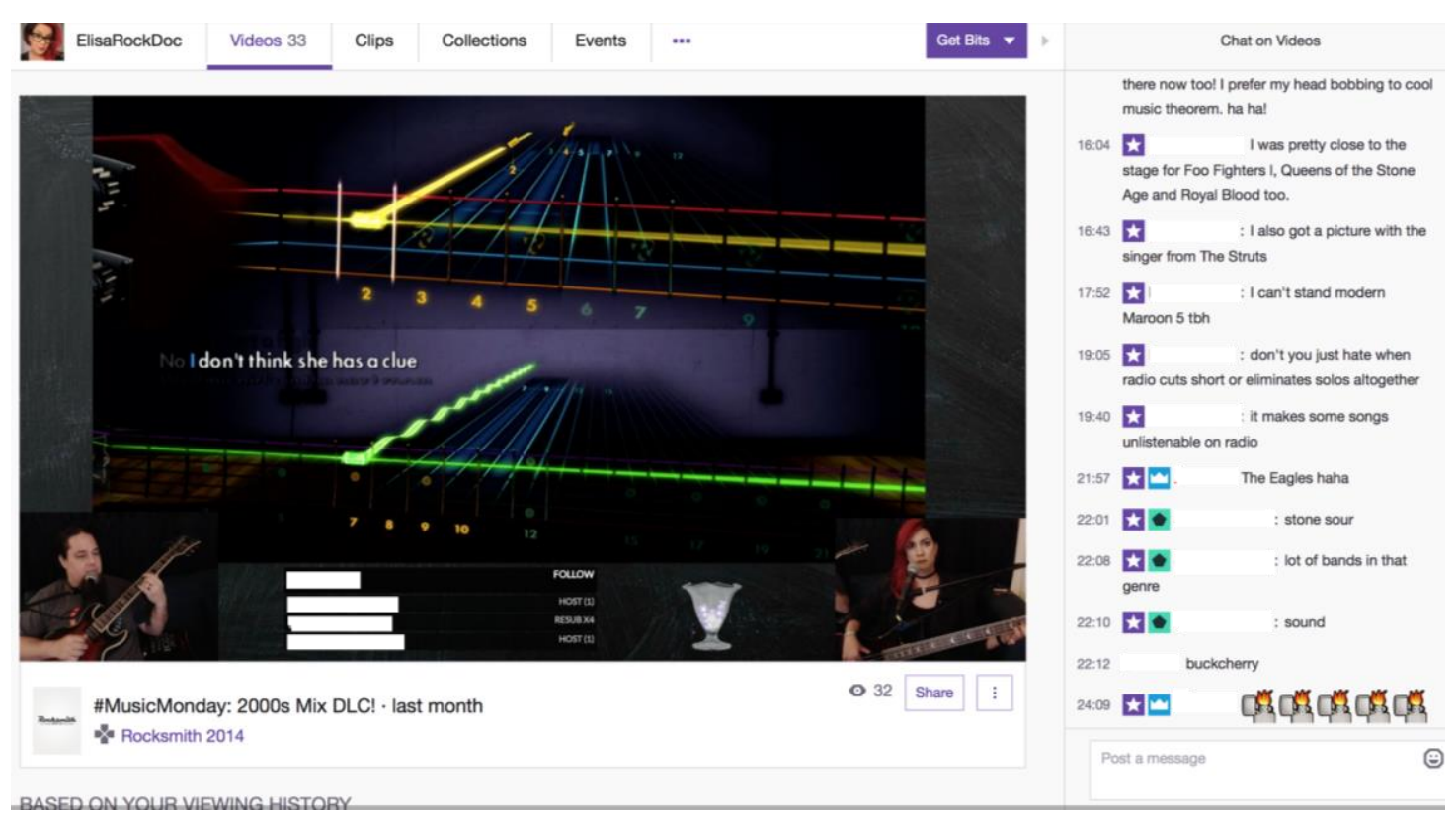

Figure 8: A typical view of the video game broadcasting site Twitch.tv. Retrieved from twitch.tv/elisarockdoc on November 10, 2017.

As a musician and overall performer, I was attracted to the prospect of building an audience for myself and my music from the comfort of my own living room, so I began broadcasting Rocksmith gameplay via the Twitch.tv service while I was in service as a Frag Doll Cadette. In order to help promote Rocksmith, the team at Ubisoft would give me redeemable codes for their downloadable content (DLC) a day early in exchange for a preview livestream on Monday nights. I would broadcast from my living room, playing the game and showing myself on camera. By previewing the DLC, I gave viewers an opportunity to make a more informed purchase while at the same time benefitting from the cultural capital of early access. I started alone, playing each song up to three times in order to showcase the lead guitar, rhythm guitar, and bass parts in each song, switching instruments halfway through. While the game's purpose was to learn guitar, and I definitely did so through repetitive play, many people in the audience wanted to see how difficult the song in its entirety was. This meant that I would have to change the song's 
settings to ramp up a song's difficulty to the maximum in order to show a complete song as opposed to playing it at my difficulty setting, which, depending on the song and the day, may only show about a quarter of the notes. With a song at its highest setting, it would often be a struggle to play it with any semblance of accuracy, which I knew wasn't the optimal viewing experience. Because Rocksmith supported multiple players, I decided to add on my guitar-playing husband to the weekly broadcasts in order to cut down on the time spent switching instruments as well as add a more-experienced player who would be able to sight-read and play the songs with more accuracy. With him on guitar, I switched to bass in order to show off the bass parts of the songs on offer each week (as seen in Figure 9):

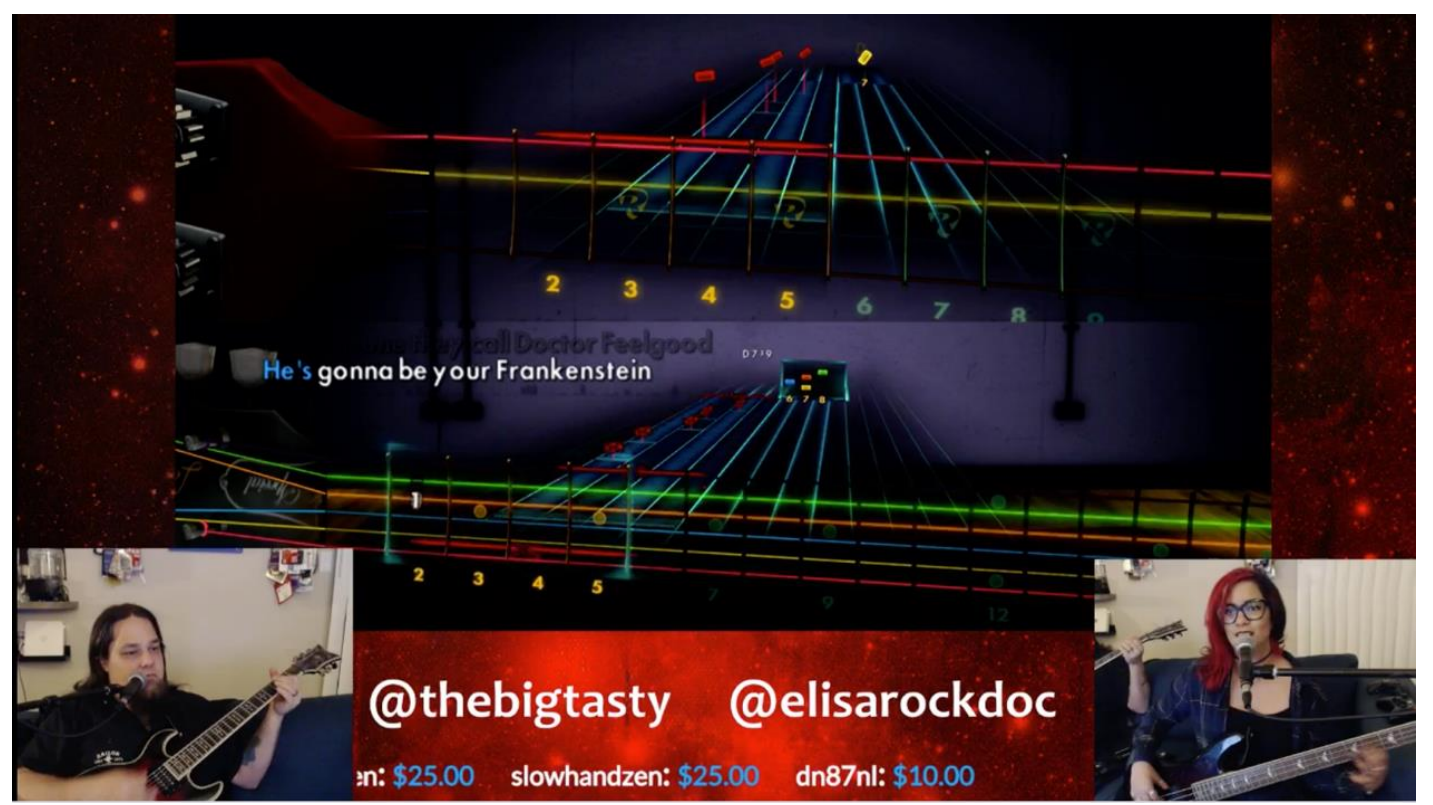

Figure 9: Researcher as performer. Retrieved from twitch.tv/elisarockdoc May 1st, 2017.

To this day, I still struggle with my choice to play bass, as well as much of the audience deferring to asking him questions about the musician's experience due to his virtuosity (he's been a guitar player for over 25 years). Having him onscreen serves the 
same purposes as my being on camera: humanizing the interaction as well as helping show the audience how to play certain parts on guitar and bass. However, despite my being the host, and the channel bearing my username, Evan tends to get more of the music questions and tasked with showing how to play certain parts.

On the one hand, I recognize that playing bass is easier for me to play than lead guitar, as many of the songs in Rocksmith have me playing more of a supportive role with recognizable music patterns that are easier for me to sight-read. Exceptions exist, of course, as Rocksmith artists such as Stevie Wonder, Iron Maiden, and Rush have deeply intricate and fast-moving bass parts that lead to hilarity as I try to keep up in a live setting. The relative ease of my bass parts compared to Evan's lead guitar parts with solos allows me to focus on both playing and interacting with the audience in the chat room without sacrificing too much gameplay. On the other hand, due to this research endeavor, I am aware that I'm potentially reinforcing attitudes towards the bass - that it is easier and a less-challenging entry point for women musicians (Clawson 1999), and that I am playing, both literally and figuratively, a supportive role to my husband, despite my name being on the virtual marquee.

\section{ElisaRockDoc, Industry Insider}

In the chapters ahead, you'll be able to see how my forays into the industry have colored the research and played a direct role in data-gathering. Experience in the industry and forging connections made it easier to identify and seek out participant observation-rich situations, and attending industry events and afterparties helped me gain easier access to developers for interviews (who tend to respond better in casual situations at a bar than a 
cold e-mail that can be buried among hundreds of others). In turn, my academic look at gender issues in gaming has affected my career in both positive and negative ways. As an example, a poolside conversation about my disappointment in Ubisoft's choice to not include a playable female character in one of their latest Assassin's Creed games turned into a writing opportunity for a major gaming website — that poolside conversation happened to be with a lead editor of said publication. This was a positive in that it granted me a (paying) visible opportunity to showcase myself as an opinion writer with an academic pedigree and make myself better-known in the wider video game industry. It was a negative in that it made me visible to members of the gaming community who wished to do virtual and physical harm to women who dared conduct feminist critique of games. I, thankfully, only experienced a brief period of harassment after the article was posted, and the harassment only occurred in virtual spaces that I could heavily moderate and curate. It did, however, make me question how it was that I made my feminist activism visible. I do not write public-facing feminist critique anymore, save for this dissertation, but I feel that I have other opportunities for activism—-mainly, by working at a video game studio.

As I write this, I am the New Media Manager at an independent video game developer and publisher. My work involves creating content for the studio on Twitch.tv and YouTube, using my experience as a broadcaster and performer to engage and educate the community on our products. I do not take for granted how it is both a big deal and increasingly not a big deal that a video game company has a woman of color as one of their prominent faces. I also handle influencer relations, making sure prominent Twitch broadcasters and YouTubers receive copies of our games in order to play them, review them, and hopefully show them to their audiences (much like I did and still do for 
Rocksmith). I try to oversample women and people of color in this part of the job, as they are often underrepresented in these endeavors. As a member of the marketing team, I review commercials and make sure they are on-brand, but I also keep an eye out and make sure that the visuals and scripts aren't going for cheap laughs at the expense of the marginalized. So far, my attempts at applying my research have been greeted with open arms - in fact, it is seen as an asset (well, for this company, at least) in an industry where feminist pop culture critique is more commonplace, as it gives the opportunity to vet some game design decisions or marketing decisions before they become public-facing blunders that could have more easily been avoided with the inclusion of a more diverse workforce.

My studio is hard at work on multiple projects. Many of them involve the use of guns, magic, and other fantastical weaponry. These games take place in barren wastes, thick jungles, the last surviving star of the universe. We cover stretches of space and time, exploring new galaxies and planet systems, as well as the drab combat scenarios of World War II. When I enter the lobby, I am confronted with multiple faces of the characters whose stories we've told: a soldier, an action-hero brute with a vulgar mouth and a buzz cut, a shapeshifting siren with red hair and glowing tattoos, a brown-skinned woman of large, muscular build. A unicorn made out of solid diamond affectionately known as "Butt Stallion." At my desk, I spin around in my chair at the mention of my name pronounced correctly — a once-rarity that has now become commonplace:

Hey, Elisa, can I get a gut check on this? Does this copy sound sexist to you? I want to make sure to be inclusive. 


\section{ROCKING OUT WITH ROCK BAND}

While not the first music game of its kind to introduce plastic instruments to American households, Rock Band (whose start screen is shown in Figure 10), with its expansion from guitar to the four iconic instruments of the genre, became my subject of study. At the time of writing, Rock Band had already reached its third main iteration, with another separate version featuring the songs and visual style of The Beatles. For the purposes of the project, however, I decided to go back to the beginning.

In analyzing Rock Band, I endeavored to answer the following research questions:

1. How is Rock Band gendered? How does it emulate or subvert gendered music or gaming discourses?

2. How are gender norms hard-coded into the game?

I hypothesized that by creating a recognizable "cover version" of the rock band participation experience via loading text, song selection, the crafting of narrative, and more, a significant portion of the sexist baggage of the music industry would be carried over. Admittedly, I was not optimistic about subversion of gender norms, and I expected that these gendered discourses would be hard-coded into the game in ways that would not make subversion possible by the player. For example, I was expecting gender selection to be a binary selection between male and female, and that avatar customization options would not allow for fluidity in gender expression and performance. I also expected that the set list would be just as lacking in a diverse canon as mainstream American rock music. While I found much of what I expected, I found some interesting answers to the research questions in unlikely places. The results and subsequent analysis of this music game classic follows. 


\section{Harmonix and Rock Band: Origins}

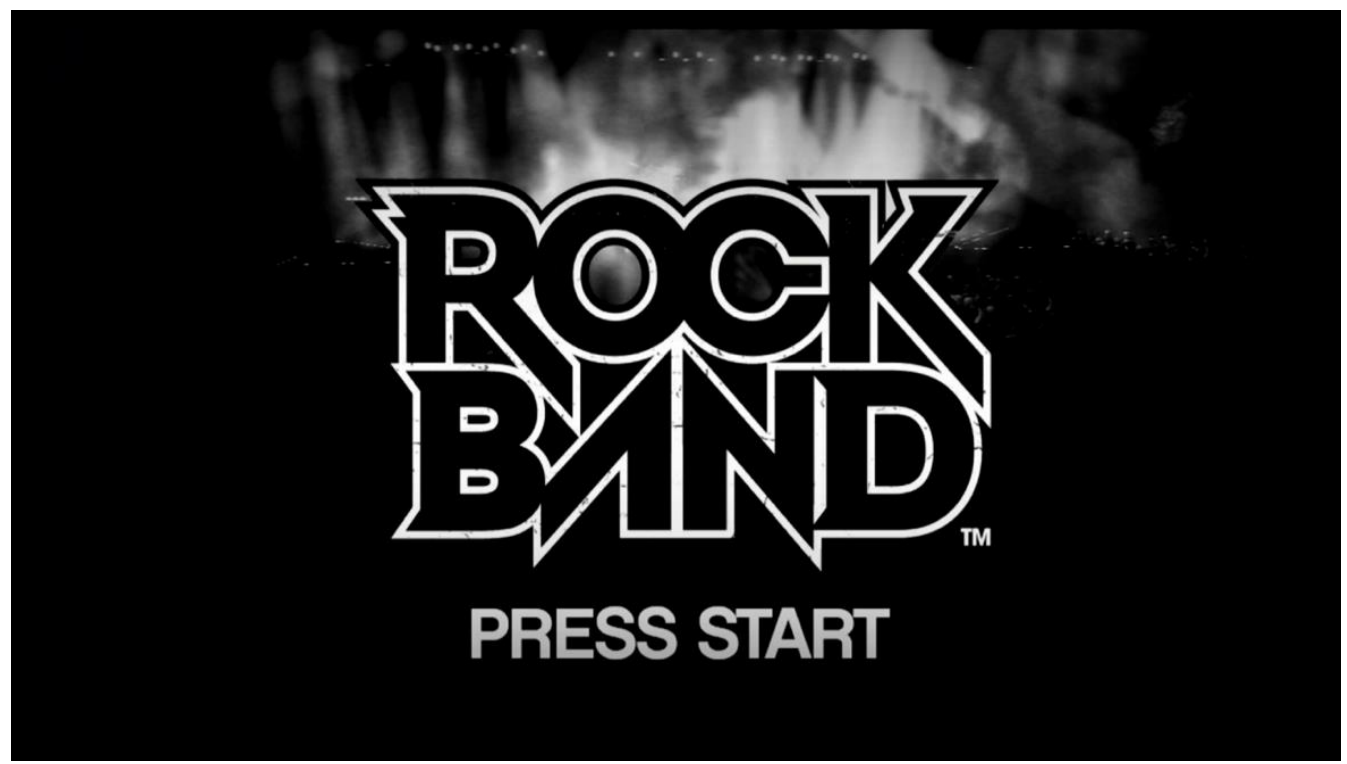

Figure 10: The Rock Band start screen. Screenshot from Rock Band.

Billed as the "world's leading music game developer" on their website, Harmonix Music Systems was co-founded in 1995 by Alex Rigopulos and Eran Egozy. Harmonix’s first foray into video games was The Axe, a game where a player could control a joystick to manipulate instruments on a screen. As "the axe" is an often-used nickname for a guitar, it is interesting to note its use in the title of a game which includes other instruments, a continuing testament to guitar's importance in the pop culture/pop rock lexicon.

After a couple of forays into beat-matching rhythm games and karaoke sing-along games, Harmonix developed Guitar Hero I and II with the publishing aid of companies Red Octane and Activision. The guitar once again becomes the central theme in their development with its roots in creating an authentic music-playing experience for nonmusicians: "If you get it right, though, the effect is that you are really playing the 
awesome guitar riff of a song you have heard a million times on the radio since you were 15 (Steinberg 2008:16).” The original Rock Band was released in November of 2007. According to the company timeline on the Harmonix website, the release of Rock Band is listed as follows: "Start a Band. Rock the World. With that simple phrase, Harmonix kicked off countless plastic bands in homes across the world, rocking out on guitar, bass, drums, and vocals for the first time (Harmonix website)." Rock Band, then, represents the evolution of the music game, adding three more core rock music instruments to the rock simulation experiment Guitar Hero began. Rock Band 2 followed a year later with the addition of new modes and new songs.

In 2009, Harmonix released The Beatles: Rock Band, "an experiential journey through the music, history, and aesthetic of The Beatles (Harmonix website)." In an ultimate display, Harmonix performed its commitment to authenticity through the visual, the tactile, and the auditory in both their game's design and the marketing. In the Harmonix company timeline, five key moments in the game's development were featured:

- Their March 2009 visit to Abbey Road Studios, where The Beatles recorded many of the songs which would ultimately become rock canon.

- Their June 2009 rooftop concert as part of a press event, "a nod to The Beatles' last public concert on the roof of the Apple Building in 1969.”

- Their E3 2009 debut, in which they were joined onstage at a reveal press conference by Harmonix CEO Alex Rigopulos, Giles Martin, Sir Paul McCartney and Ringo Starr, Olivia Harrison, Dhani Harrison, and Yoko Ono Lennon. 
- Joining The Beatles' frontman Paul McCartney on his Summer 2009 tour, giving attendees an opportunity to play the game at various tour stops.

- The game's release in September 2009, heralded by The New York Times as "the most important video game yet made (Schiesel 2009)."

In an effort to deliver the most attainable simulation of The Beatles, Harmonix borrows from the places and the people that made the music happen, rather than venturing a guess. In the rest of this chapter, I assert that this attention to detail in order to produce a more accurate simulation was not a special case, but it is a thread of intent carried through the development of the original Rock Band and the games that followed. I also aim to show that, in an effort to reproduce the feeling of playing in an American rock band, the gendered baggage of American popular music tends to follow, and it shows in places like song availability, loading screen text, and avatar customization. However, this does not mean that the developers are not conscious of their role, as you will see in the following section featuring interviews with members of the Harmonix development team.

\section{Harmonix Speaks}

After developing a relationship with developers through the Harmonix community team, I was able to secure interviews with three developers that had a hand in the creation of Rock Band, including folks involved in the creation of the plastic hardware peripherals that accompany the game as well as narrative design. The office itself resembled what I pictured a video game development office to look like: rooms filled with desks, computers, and the occasional arcade machine and other whimsical touches such as an entire autograph room where visitors could sign their name and draw 
pictures (the company has since moved offices from Cambridge, MA to downtown Boston).

The interviews took place in a conference room, where I spoke with an audio director, a narrative producer and writer, and a game producer over the course of an afternoon. A primary theme throughout the interviews was an emphasis on authenticity to the rock music experience, whether that was through the makeup of the studio, development of hardware, narrative elements in the games themselves, or song choice. Describing the studio's demographics, the producer asserted a balance between the goal of producing video games while maintaining an authentic experience through hiring: We have a lot of Berklee grads on staff, but you know I'm not a professional musician and nobody working here is. We are professional video game makers, that's what we do...I would guess in terms of people that own their own instruments and sort of play around when they can, I would say 70 percent, 60 percent. More than half (Developer 1, Harmonix).

Familiarity with instruments contributed to a more authentic simulation of playing through the design of the game itself. Guitar Hero and Rock Band's design translates songs played with a variety of instruments into a five-lane rhythmic highway. After working with record labels and musicians to obtain the files (or even tapes) of individual instrumental tracks, it would be the work of in-house musicians to transcribe the instruments into a playable format that still feels like you're playing "real music." The next step is to distill that by difficulty, so that by the time a person is playing on Expert Mode, people are playing "at least, rhythmically, note for note of what was really 
happening." The fidelity extends from the note-for-note fidelity on screen to the actual performance portion, including the feeling of your fingers on a plastic guitar (note that, unprompted, interviewees tended to reference the guitar more often than the other instruments available to play in the game). On playing the guitar in particular, the audio director noted inclusion of intangible elements that could only be achieved by development from personnel with experience with the "real thing:"

We sort of developed these kinda of semi-written down, half-unwritten rules of what we do. We try to have power chords on (buttons) one and three because that's kind of how you play them on a real guitar, we try to save stretches for one type of thing... We just sort of like figured out after doing a few games that this seemed to lay nicely and feel the most like you're actually really playing the instrument even though you're playing 5 buttons on a plastic guitar... (Developer 1, Harmonix).

They reiterate that having people within the culture contributes to a general "feel" of authenticity that is not achievable otherwise. The employees that are musicians are socialized into the culture so as to have an alleged common understanding of their subject group:

We can say [the rules are] half-written down because they weren't things that you have to sort of explain, these people sort of instinctively knew how that feels and, 'Oh this doesn't feel right' 'cause they kind of do it for real (Developer 1, Harmonix). 
The goal of having musicians on staff was to ultimately achieve bringing music and the experience of playing music to the non-musician. The feeling of playing extended from the translation of songs into the in-game design to the physical hardware that helps "sell the experience." By having a plastic instrument, you could reach a level of simulation that a regular console controller or a mouse and keyboard could not provide:

...you could do all the things you could do when you're playing... you could get in the groove, you just feel the music and you could tell when you hit notes and when you don't. It just felt like you were really kind of doing it in this weird abstract way (Developer 1, Harmonix).

The team also took steps to inject authenticity into the plastic instruments through relationships with actual instrument manufacturers, which appear as sponsors within the game (as seen in Figure 11) as well as consultants for the hardware itself.

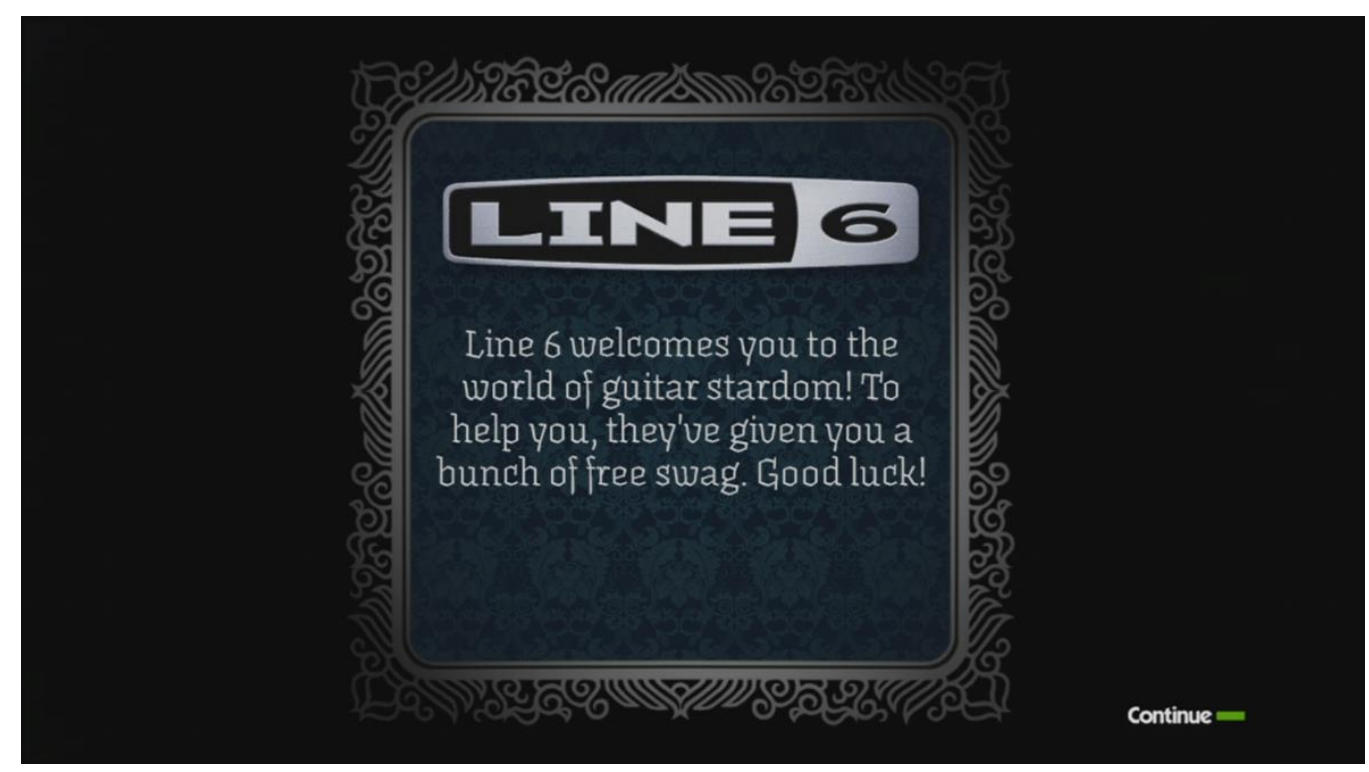

Figure 11: Real-life sponsors for a virtual band. Screenshot from Rock Band May 1st, 2017. 
The producer I interviewed commented on working with instrument manufacturers such as Fender, Gretsch, Rickenbacker, etc., but also running into having to make concessions (particularly on drum hardware) due to the restrictions of game design and fitting hardware into people's living rooms:

The drums were harder because we had a lot of designs. You start with a paper drawing and it's an impossibly wide possibility space, which makes it look like anything. And then you start to get into manufacturing constraints. How are we going to build this, and how are we going to package this? ...We were really thinking about how, what materials do we want, what does it sound like when you hit that pad with the drumstick...how stable are they, what do these look like in a person's living room, and are they going to stay there forever (Developer 1, Harmonix).

Aside from hardware and level design, I was particularly interested in the narrative elements of the game, the story of rock and roll they told through text. The narrative producer and writer lent quite a bit of insight into the process, as well as insight into how gender factors into her work (she was the only woman I interviewed on the development side for either game). A musician in her own right, she injected a lot of her and other musicians' experiences into the text, whether that be loading screen tips or descriptions attached to clothing and accessories for your rocker avatar in order to lend another degree of authenticity to the game:

I had [to make] the sort of random tip number 326 and it would just be like some joke and it was always based off some fight I had the night before with my band, 
so it was just me venting and making fun of myself about the different roles that different people in bands tend to play, and typical experiences that you have on tour and being broke, that kind of thing. That's where most of those came from (Developer 2, Harmonix).

A more in-depth discussion of the in-game text and what themes regularly appear follows later in this chapter, but it is important to take note of the intent behind the narrative, courtesy of the writer interviewed. When I filled her in on my inspiration for conducting this dissertation research, a loading screen tip from a Guitar Hero game that stated, "When in doubt, blame the singer's girlfriend," she looked visibly disappointed—like it had been a potential failure on her part. In fact, that loading screen tip seemed to go against her current intent when working on Rock Band games:

...if I wrote it, it probably in the first few years of my career. Cause now I would never do a gender thing now. I don't think there's ever been a pronoun or an assumption about gender. We're really hardcore. We keep the company extremely hardcore about it (Developer 2, Harmonix).

That it was an explicit goal for the company, or, at the very least, for her, to make sure that narrative choices and game design are gender-inclusive was heartening. The reason for her maintaining that level of awareness stemmed from her own experiences being a woman playing a game, playing as a female character, and then being broken from the immersion when the game refers to her character with a he/him pronoun. She aims to make sure the company doesn't make those same mistakes: 
I think this company is pretty good in general about watching out for anything, but it's on the forefront on my mind, so it's easier for me to catch them. No one has ever accused me of being too hardcore with that. But there's little things-like in the example of the game that I'm working on now... we wanted to give you an achievement like "Maestro," and that's gendered, so they were like "Maestro?" and I'm like yep, sorry. So, I'm sure I've missed a few over the years, but it's definitely something we look out for (Developer 2, Harmonix).

As mentioned, while the intent is there, it is possible that the team "missed a few [nongender inclusive nods] over the years" of development. The following portions of this chapter examine in what ways exercises of authenticity in musicianship also become exercises and performances of gender through song choice, narrative, character customization, and in-game cinematics.

Rock Band: The Set List

According to the developers interviewed at the Harmonix offices, song choice in the game is a result of a number of factors, including general appeal to the audience as well as variety of genres and decades:

Our goal in Rock Band has always been to kinda appeal to a wide variety of people. So, we would always want to...our goal is to try to end up with a good mixture of songs from all of the rock decades from the 60 s from the present... and a good mixture of styles in the kind of rock thing (Developer 3, Harmonix). 
Other factors include how songs fit into the existing catalog so that there is, "a good mixture of fast and slow songs," as well as a consideration of whether a song can be easily supported by the existing peripherals (guitar, bass, drums, vocals, and later keyboards). While the focus and intent mirrors the variety and authenticity inherent in other parts of the game's development, it is worth exploring whether the intent to replicate a variety of successful popular music/rock songs results in a predominantly male-musician list of options, or whether the song list can represent a subversion of or a departure from the rock god pantheon.

In order to examine this, I conducted two studies investigating the gender makeup of the artists available for people to play in Rock Band. The first was a pilot study performed in the fall of 2010, using a simple random sample of 200 songs from the Rock Band Network. The Rock Band Network is the list of songs that is both available on the game disc upon purchase and songs that people can purchase and download onto their systems to play. Using the coding system and quantitative analysis detailed below, the study revealed significantly fewer women than men as artists, as well what appeared to be a correlation between female presence in a band or as a solo artist and a song being rated a lower difficulty level with the use of a simple Chi-square quantitative approach. Figure 12 shows the significant results (with a P-value less than .05) when looking at the instrument and difficulty rating: 


\begin{tabular}{|l|r|r|} 
Variable Relationship $\nabla$ & Chi-Square & P-Value \\
\hline FemPres/OneStar & 6.488 & 0.011 \\
\hline FemGuit/OneStar & 6.342 & 0.012 \\
\hline FemBass/OneStar & 10.89 & 0.001 \\
\hline FemBass/TwoStars & 4.538 & 0.033 \\
\hline FemDrums/OneStar & 12.555 & 0.00 \\
\hline
\end{tabular}

Figure 12: Chi-square results when looking at Female Presence, instrument use, and song difficulty (Retrieved from XLSTAT November 2011).

As seen above, there appeared to be a correlation between female instrumentalists and a lower difficulty level, while none was found for vocals. The study concluded that it was possible that this relationship stemmed from existing attitudes about women as instrumentalists versus vocalists that may have infiltrated the design of the difficulty mechanic, but that more research needed to be done as to how difficulty was ultimately assigned before concluding anything definite. The following represents the expansion of the pilot study, taking all available Harmonix-produced tracks within a certain time period as a population rather than focusing on a smaller sample size.

\section{Definition: female presence and role}

The songs were first coded for whether or not the band featured any female presence in its primary members $(0=$ failure, $1=$ success/female presence $)$. While it is possible for artists to employ the use of background vocalists or auxiliary instrumentation in the recordings, the primary band is made up of members likely to be consistent in live performances, studio recordings, and the public face in marketing and promotional materials. Country artist Carrie Underwood, for example, may have female 
instrumentalists on her recordings or live performances, but she is the primary and only artist coded here.

Once the presence of a female primary member was coded, the data was further coded for which role the female plays in the band. Bands were coded as having Female Vocalist, Female Guitar Player, Female Bass Player, or Female Drummer. These categories were picked to the exclusion of keyboard players, percussionists, or other instruments, primarily because these are the only instrument peripherals available for players to use in the game itself. In cases where the lead singer is also responsible for an instrument, both roles are considered and coded for: one example is Orianthi, a solo artist, who is both the lead singer and guitarist on her track. She is coded as both singer and guitar player.

In a few cases, songs appear in which the artist is from a fictitious work (television, video game, etc.) or the musicians themselves represent themselves with animated avatars (as in the case of Gorillaz). For the purposes of this study, the gender coding of the artist is based on the corporeal musician. For example, GLaDOS is a fictional artificial intelligence from the Portal video game franchise. GLaDOS has a female voice and is portrayed by actress and singer Ellen McLain. Since McClain provided the vocals for GLaDOS's "Still Alive," the song is coded as having female presence.

\section{Defining difficulty}

The songs are judged by the game developers as rating anywhere from "No Stars" (the least difficult), to "Devilish," the most difficult (one step above Five Stars). For 
coding ease, the Devilish rating was changed to Six Stars, making the difficulty rating a seven-point scale. While each song has an available measure of difficulty per instrument, the study was only concerned with the overall difficulty awarded to a song. For the pilot study, difficulties were coded as female presence was, as dichotomous variables. For the dissertation, difficulties were recoded as an interval variable on a seven-point scale from 0 to 6 .

\section{Rock Band revisited}

As of May 9, 2013, there were 4,257 songs available to play from the Rock Band Network. Out of those songs, 2,119 entries were created by users that had the software and tools to create the custom content. Because of time constraints in investigating the band demographics for these songs, they were eliminated from the population, bringing the total number of songs to 2,138 . The total breaks down as follows:

- Rock Band: 58

- $\quad$ Rock Band 2: 84

- Rock Band 3: 83

- LEGO Rock Band: 45

- Green Day Rock Band: 44

- Downloadable content: 1,824

LEGO Rock Band and Green Day Rock Band songs were removed in the interest of both time and possible redundancy. Harmonix provided a list of songs and associated difficulties that limited the population to songs from Rock Band, Rock Band 2, Rock 
Band 3, and downloadable content. After eliminating duplicates, the population became an even 2,000 songs.

Descriptive statistics show that $16 \%$ of songs $(n=279)$ had any female musicians using the core instruments of the Rock Band franchise (guitar, bass, drums, and vocals). While the set list did feature key members of "women in rock" canon such as Jefferson Airplane and The Go-Gos as well as bands with women developers from Harmonix, the $16 \%$ does appear disconcerting at first blush. Despite the attempts at inclusive narrative design and potential means of gender script subversion in the form of clothing/makeup options, the game's commitment to authenticity ends up hard-coding and presenting a mostly male genre.

The lack of representation is replicated in general, and it is also replicated per instrument (Figure 13). Out of that meager 16\%, 20.4\% $(n=57)$ were guitar players, $19.7 \%(\mathrm{n}=55)$ were bass players, $8.9 \%(\mathrm{n}=25)$ were drummers, and $50.8 \%(\mathrm{n}=142)$ were vocalists:

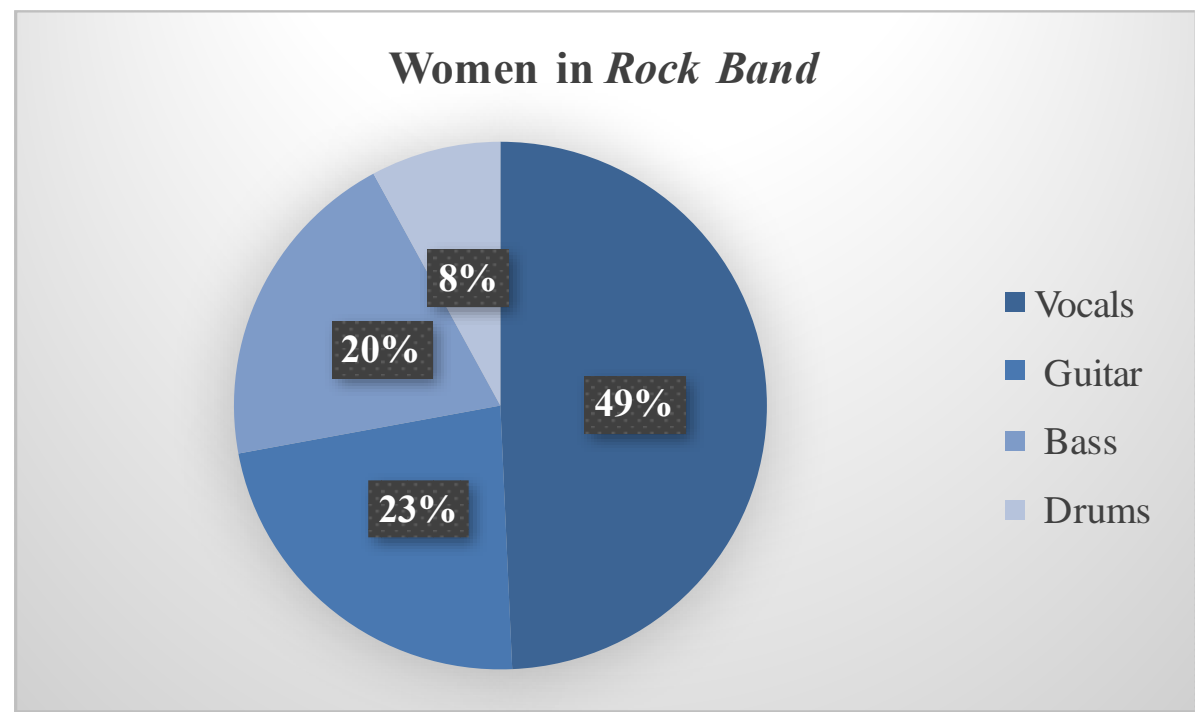

Figure 13: Representation in the Rock Band set list. Image rendered in Excel. 
As feminist musicologists (Garr 1992, Clawson 1999, Walser 1993, et al.) have observed, instruments are often gendered, with women being primarily labeled as vocalists, guitars being imbued with phallic symbolism, and the use of one instrument over another being directly linked with a band's perceived masculinity and sexuality:

An interview with Charlie Benante, drummer in the thrash metal band Anthrax, confirms that...the use of a feminine-coded instrument in the context of heavy metal evokes the specter of homosexuality. When an interviewer asked, "Would you ever consider using keyboards as a major part of the song? Benante replied, "That is gay. The only band that ever used keyboards that was good was UFO. This is a guitar band (Walser 1993:130).

In one quote, you see the conflation of instrument choice, masculinity, heteronormativity, and virtuosity, which partially explains some of the attitudes behind a perceived "lack" of women musicians in mainstream American rock music. With women as majority vocalists in Rock Band, one could say that the game then provides a faithful replica of rock music. On the other hand, with such a focus on making the narrative portions of the game more gender-inclusive, the song list represents a missed opportunity to subvert norms (in particular, the norm of the woman as absent of guitar or drum virtuosity in rock music) and oversample women musicians in the service of introducing players to more of them.

Much like the previous sample study, this more comprehensive version turned its eye to difficulty. The songs were coded for their difficulty level, ranging from zero stars to six stars, also known as "Devilish" difficulty. As seen in Figure 14, difficulty is 
distributed non-normally, with songs skewing easier (one or two stars) and mid- to upperlevel difficulties distributed almost equally:

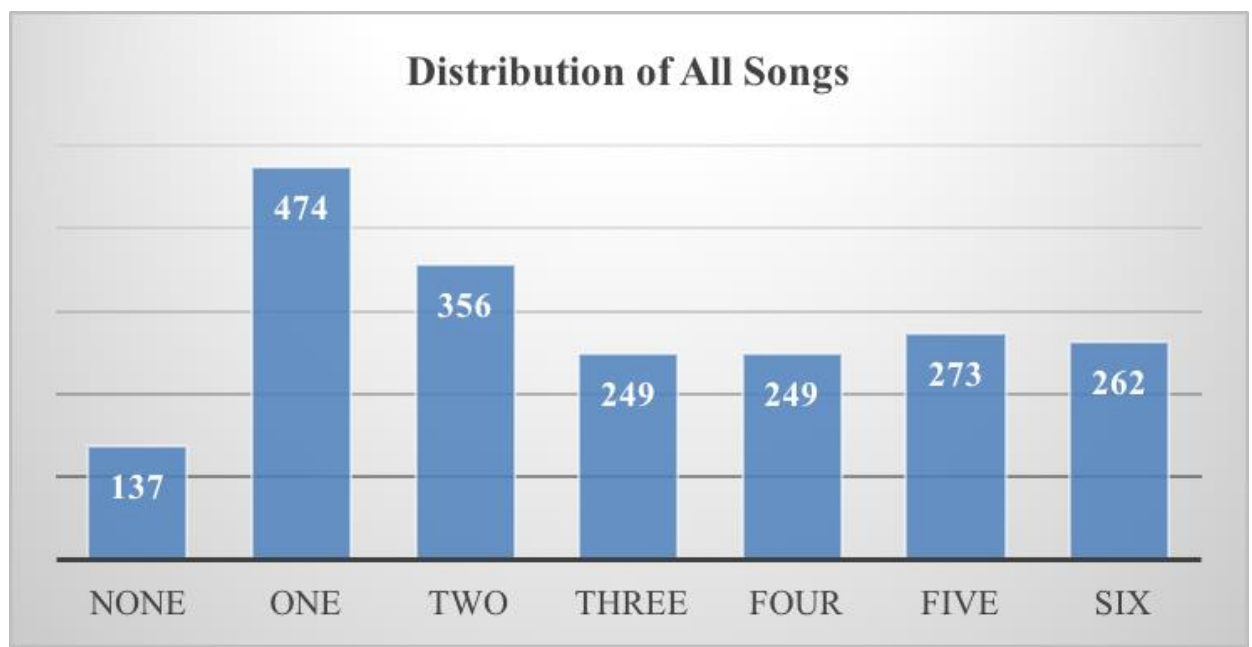

Figure 14: Distribution of difficulty levels in Rock Band in number of stars. Image rendered in Excel.

When looking at the distribution of songs featuring bands coded with having men only

(Figure 15), the distribution appears similar to the overall pattern:

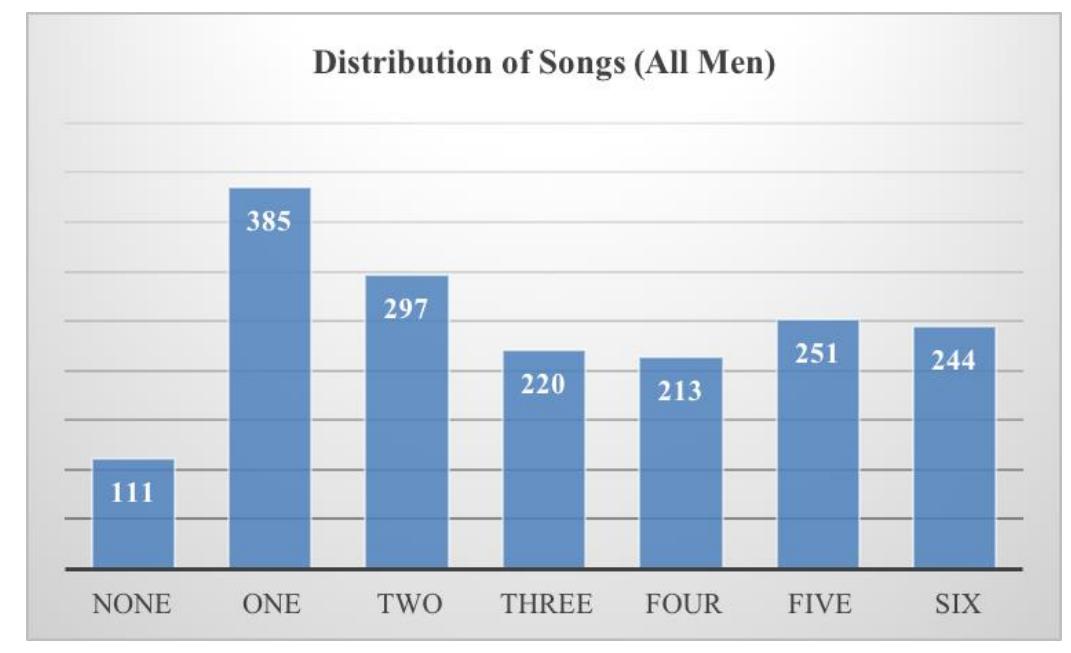

Figure 15: Distribution of difficulty levels among songs which do not feature women musicians. Image rendered in Excel. 
When looking at the distribution for songs featuring any woman in the band (Figure 16), the distribution shows an interesting shape:

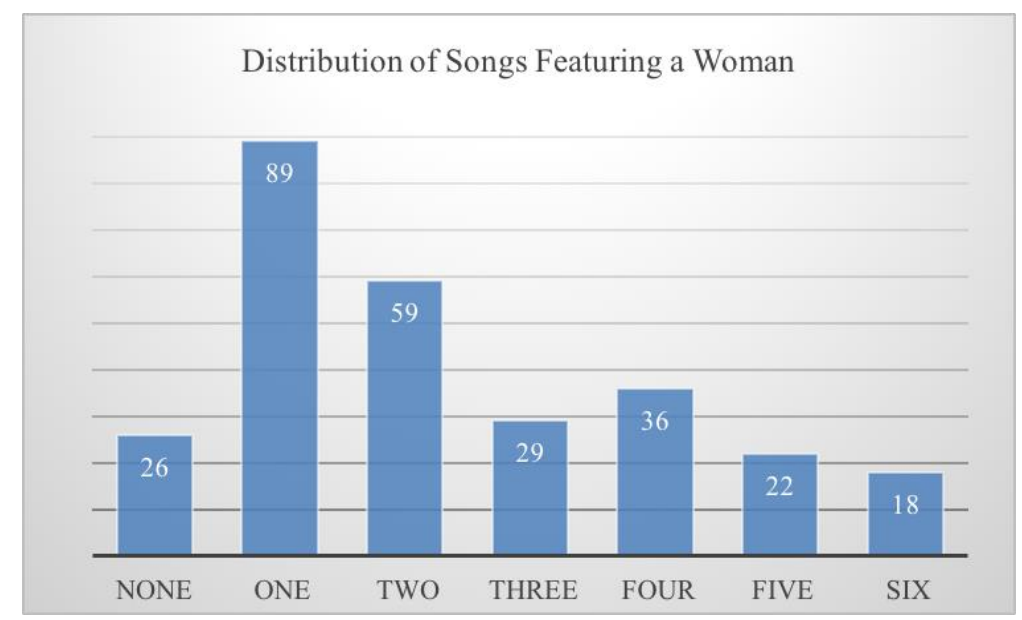

Figure 16: Distribution of difficulty levels among songs in the overall Rock Band song list which feature a woman musician. Image rendered in Excel.

The left side of the distribution is consistent with the other two, but there is a spike in four-star difficulty songs that is not present in the other two distributions. While it may be heartening to see songs featuring women as having that spike in difficulty due to the perceived virtuosity required to excel at them, only $30 \%$ of the songs $(n=11)$, however, feature women instrumentalists.

When it comes to the higher difficulty levels of 5 and 6 stars, there is a lower percentage $(14 \%)$ of 5- and 6-star songs featuring women $(n=40)$ than there are for men ( $28 \%$ ), with the overall percentage of harder songs as holding $26 \%$ of the set list. Could this discrepancy result from the gender makeup of the band? It's possible. Gillian Garr's (1992) opus on the history of women in rock features countless tales of women musicians not being taken as seriously as their male counterparts. Nancy Wilson, guitarist for Heart, notes the trade-offs of having notoriety: "largely, you don't get taken seriously; that's the 
downside. Nobody believes that you're an artist or that you can play that well or that you are legitimate to begin with (186)." Tina Weymouth, bassist for Taking Heads describes treatment of women musicians in the press: "Women musicians tend to be treated by critics like women drivers...if they aren't much good, well what can you expect? And if they're hot stuff, it is despite the fact that they are women (212)."

Women aren't the only ones affected. It seems that men that opt to add femininecoded accessories to their makeup or wardrobe add a loss of musical credibility along with them. Robert Walser, in his look at androgyny in metal, points out how surprised he and other fans are at discovering the virtuosity of bands that choose to wear makeup:

Fans link visual signs of androgyny with an abdication of metal's usual virtuosic prowess. 'It seems like if you have the makeup you're thought of as less than a musician,' complains Poison's guitarist C.C. Deville (128).

However, difficulty in rock music video games is not a one-person decision, but the average of opinions from designers as well as multiple quality assurance personnel focused on the balance and "feel" of the game. On the one hand, this can point to the difficulty numbers being a mere coincidence. On the other, it can point to how ingrained and systemic these attitudes towards women musicians are.

\section{Rock Band: Figurative School of Rock}

Rock Band is more than just miming songs. The franchise aids in the conversion from player to performer through its narrative. The use of loading screen tips, postperformance announcements, and "flavor text" for avatar customization options weave a narrative of player-as-rock star that traffics in authenticity through allusions to rock 
music history, songs, and artists. The narrative producer I interviewed at Harmonix was gracious enough to provide the entire text of the original Rock Band video game, including loading screen tips, venue names, and flavor text accompanying clothing and accessory options. I conducted a discourse analysis of the text, coding each entry in MaxQDA to generate categories of content.

Of sound guys and Yokos: loading screen text

Loading screen text appears when moving from one portion of the game to the other (i.e. from song selection to entering the level and playing the song itself). Figure 17 shows one such example:

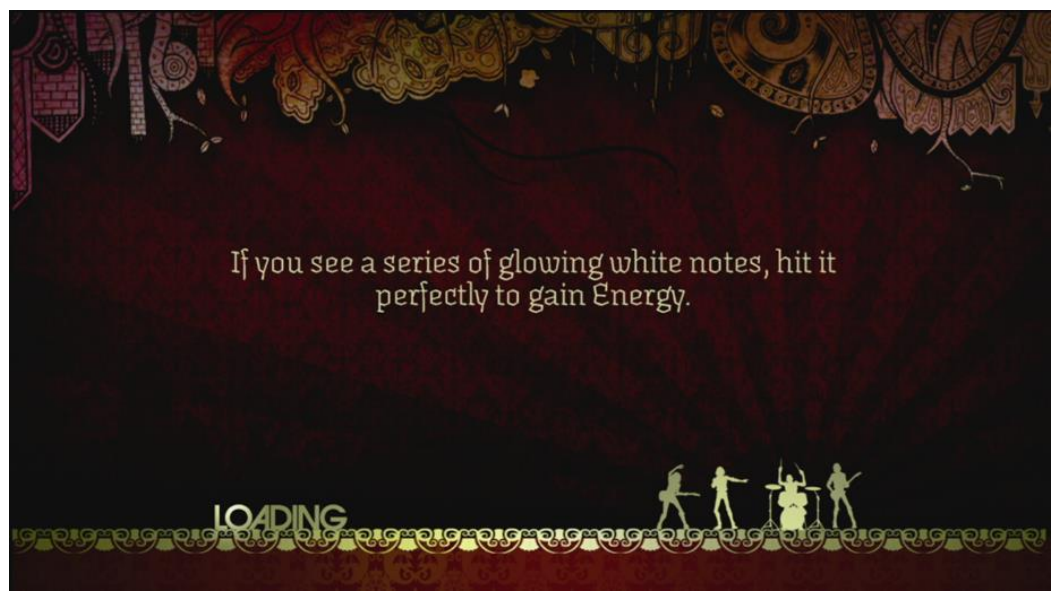

Figure 17: Loading screen text with game tips. Screenshot from Rock Band. Many tips, such as the above, focus on how to play the game, including proper audio setups or pointing out available modes to play:

- If you get a long enough streak on Bass, you'll start a Bass Groove and get up to a $6 x$ score multiplier!

- The little circles are percussion notes. Hit your microphone like a tambourine to play them. 
- Some TV and home theater setups can create a delay between what you see and what you hear. If the gameplay seems out of sync, select Calibrate System from the Options Menu to fix the lag.

The most numerous kind of loading screen text is what is described as "loading trivia." Out of 375 coded segments of total loading screen text, $73 \%$ of them $(n=276)$ referenced musicians. This makes sense, as the writers specifically target attaching loading tips to the songs and artists that are about to be played. The process, as explained by the writer I interviewed, is as follows:

... [It was] me or one of the other writers researching bands or throwing out stuff we knew. We had to deal with a lot of legal approval, but it was really fun to find that stuff out. It was kind of fun when you get maybe some awkward artist that isn't your thing, but you now have to come up with 5 interesting things about the song and you don't know about the song, so we inevitably [find that] 'there's this lyric that's kind of interesting and it turns out to be about this' and so then you have a little tip (Developer 2, Harmonix).

Out of those 276 loading screen tips, only $14.5 \%$ of them $(n=40)$ referenced any female artists. This is relatively consistent with the $16 \%$ of songs featuring women found in the quantitative analysis. Even so, those female artist numbers can be whittled down further when you consider that some of their appearances are attached to an all-male band's loading screen tip, sometimes referencing women in terms of their relationship with men:

- In 1998, R.E.M.'s Michael Stipe published a book of his photographs of influential singer/songwriter Patti Smith on tour. 
- Jet named their band after a song by Paul McCartney's band Wings [which featured keyboardist and singer Linda McCartney].

- Bon Jovi guitarist Ritchie Sambora used to date Cher.

- Ozzy Osbourne's wife Sharon was briefly the manager of the Smashing Pumpkins.

The tips are not only factual in their content, but they're reminiscent of women's roles in rock music as a whole - in rock music culture, women are described more often than not in relationship to a man. Donze (2010)'s case study of a rock club showcases how women are either girlfriends, wives, groupies, or potential groupies: “...fan personas are constructed mainly through heterosexualized roles (260).” On the artistic front, women musicians are described in relation to their male counterparts, whether that's as concrete as the band Heart being referred to frequently in the press as the female Led Zeppelin, or in more abstract terms, as in the applying of a female-gendered adjective to a musician signifier (according to Feigenbaum 2006):

Adjectival gender markers and gender binaries also work to support an authentic/inauthentic dichotomy that devalues women. This type of distinction often precedes a non-gender specific noun, such as, 'the girl rocker,' or 'a chick singer (40).'

I would argue, however, that the "non-gender specific noun" becomes a gender-specific noun as soon as the girl/chick adjective is added. A singer, guitarist, bassist, or drummer is a man until specified otherwise, much like "girl gamer" became a necessary label for women who play video games (regardless of age). When women appear in loading screen 
text more often than not because of their relationships, players receive further affirmation that women's role is on the sidelines in rock music.

Another kind of loading screen text has to do with another set of people on the sidelines: managers, sound engineers, roadies, bodyguards, and PR managers. These ancillary characters appear in loading screen text during the game's story mode, which tasks you with playing pre-determined sets at different venues. The sets get progressively harder, and as you attain levels of success, your achievements are marked by rock culture milestones. These milestones are made to emulate a typical journey in the life of a rock star, where you start with a van, upgrade to a bus, and acquire an entourage of management and other ancillary characters to denote success. You get roadies to help move your gear for you, earn bodyguards for protection, or hire a PR firm to tidy publicfacing messes. When looking at the loading screen text attached to members of your band's entourage, it becomes almost remarkable how little they rely on gendered pronouns as shorthand, particularly for the manager:

- You got a call from a shrewd manager who wants to help make you a star! You now have an experienced manager who can help get you booked at bigger clubs! Nice work!

- Your new manager can now book you in nearby cities, and your new van gets you and your gear there! Keep playing more gigs so that your manager can book you at bigger venues and cities.

- Congratulations! You've added a MANAGER to your entourage! As well as booking gigs, you can call your manager anytime if you want advice on where to play next. 
The repetitive use of "your manager" versus "he/him," "she/her," or even singular "they/them" could be an attempt at maintaining a sense of gender neutrality. This could also be based on having both male and female managers in mind — the previously mentioned loading screen text referencing Sharon Osbourne as the Smashing Pumpkins' manager (and knowledge of her increasingly public-facing role as Ozzy's manager in the wake of their reality show) shows an awareness of women managers. Unfortunately, not every person you can add to your crew received the same treatment. The bodyguards are once referred to as "really giant dudes with crew cuts," and the person responsible for the band's sound at a show—-the sound engineer—slipped through the cracks in a significant way, consistently being referred to as a "sound guy:"

- Everybody has an off-night, even you! When that happens, it sure would be nice to pass the blame... and that's what the SOUND GUY is for! Play well at this gig and you'll end up with an audio pro who can handle your sound AND your reputation!

- Keep an eye out for a SOUND GUY who can maintain your signature sound at huge shows.

- Sweet, a SOUND GUY has joined your entourage! Now you've got a professional handling your sound, so you'll always have somebody to lay the blame on. At this level of success, you might find yourself needing BODYGUARDS soon...

According to audio advocacy organization Soundgirls.org, only 5\% of professionals in audio and musical production are women (soundgirls.org). As with so many other tech fields, a lack of role models could be partially to blame: women don't see themselves in 
the industry, and it signals to them that they may not be suited for that line of work. As the music industry is about networking and connections, a homosocial workforce will self-perpetuate itself if people don't go outside their existing networks. Seeing a character labeled a "sound guy" is one more data point confirming and normalizing existing biases.

\section{The magic's in the makeup: clothing, accessories, and makeup text}

Rock Band gives you the opportunity to earn money by playing shows in its World Tour mode. This money can be used to buy customizations for your character in the form of hairstyles, facial hair (for men only), clothing, accessories, and makeup. The reference sheet I was given with all of the text in the game reflects the sex divisions of customizations in interesting ways. Wrist accessories and earrings appear to be gender neutral. Hair, makeup, and clothes are divided by gender. Facial hair is only available for men-which is interesting to note now that more modern games with character customization (The Sims 4, Dragon Age: Inquisition) are embracing more robust and inclusive systems that allow options such as facial hair, Adam's apples, haircuts, and makeup options available to every character regardless of sex designation. To wit, looking back at text from a now ten-year-old game in the wake of this much progress leaves Rock Band looking more like an early pioneer with tons of unfortunate baggage to work through rather than an exemplar. 
While makeup, clothes, and hair options are divided by avatar sex, there are often times where male and female avatars have the same options (see Figure 18):

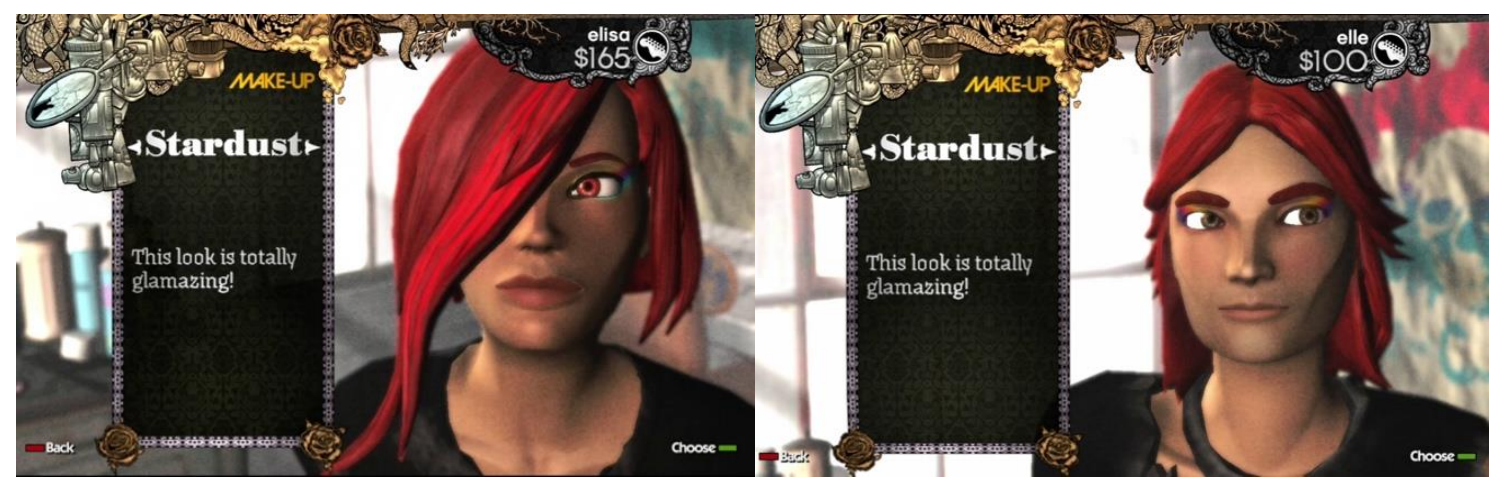

Figure 18: A makeup option, Stardust, which has the exact same look, name, and descriptive text for both male and female avatars. Screenshot from Rock Band.

The shared availability makes sense in the branded clothing category, where your avatar can wear shirts featuring major instrument brands. This linkage between prominent instrument brands and their video game counterparts is a two-way street. Rock Band can use their presence as a way of injecting even more rock n' roll authenticity into the game, and the instrument brands benefit from the brand recognition that might happen if and when a player chooses to trade a plastic instrument for authentic (which becomes more of a possibility with the introduction of "pro" playing modes in later games). By rendering these pieces as, essentially, "unisex" or "androgynous," one can gather that this opportunity to subvert existing gender norms can be seen as progress.

Androgyny in rock music, as described by Robert Walser (1993) is, "the adoption of male performers of the elements of appearance that have been associated with women's functions as objects of the male gaze (124)." This definition of androgyny has a long-standing history in the rock music subculture. Recent examples include the emo subgenre's penchant for what is known colloquially as "guyliner," while historical 
examples include artists like David Bowie and the entire hair metal/glam metal subgenre, where bands are known for their loud, exaggerated makeup and (over)use of hair products to set their hair sky high. Looking at glam metal and rock in the age of the music video provides examples of the negotiations between application of feminine trappings and portraying heteronormative masculinity. Walser continues his exploration of androgyny in rock and metal with a look at Van Halen's lead singer, David Lee Roth, who was noted for his martial arts practice in addition to his high voice and flamboyant looks:

David Lee Roth self-reflexively connects his enthusiasm for bodybuilding and martial arts training to his "feminized" image on stage: "A lot of what I do can be construed as feminine. My face, or the way I dance, or the way I dress myself for stage...But to prove it to myself, to establish this [his masculinity], I had to build myself physically. I had to learn to fight (129).”

In the effort to replicate aspects of the rock music industry, Rock Band retains accuracy in the inclusion of clothing and makeup options that allow for the creation of androgynous rockers such as David Lee Roth and others in the glam metal scene. However, a lot of the gender negotiations that tend to occur as a result do not tend to appear in the Rock Band franchise - the games are typically rated by the Entertainment Software Review Board (ESRB) as T ("Teen") because of the lyrical contents of the songs as well as "mild suggestive themes," so the excess of drugs and groupies and other heteronormative compensatory behavior that can come with a tube of lipstick would not be found here. 
There are occasions in which men and women avatars can share the same piece of clothing or makeup, but there is different text attached. In the below example, the women's and men's corduroy pants are similar in color and shape, they appear under the same genre of clothing ("Rock Rags"), and their listings are the only clothing items with "cords" in the descriptor. They are, however, described differently (see Figures 19 and 20):

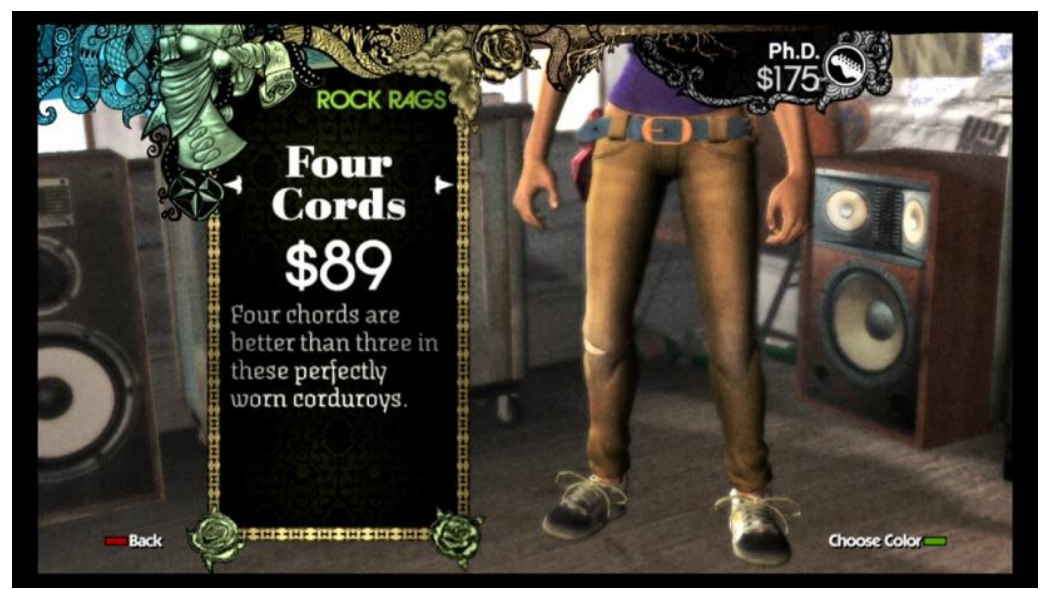

Figure 19: Women's corduroy pants. Four Cords: Four chords are better than three in these perfectly worn corduroys.

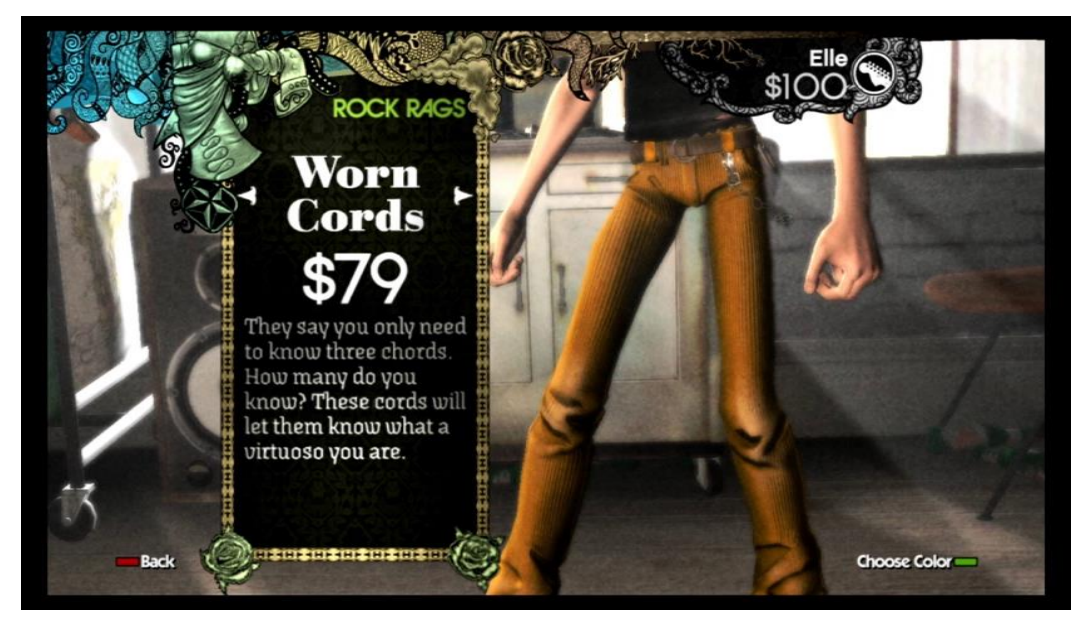

Figure 20: Men's corduroy pants. "Worn Cords: They say you only need to know three chords. How many do you know? These cords will let them know what a virtuoso you are." 
While women are limited to four chords and a sense of finality, men are both given an open-ended possibility of chords and have the added benefit of being called virtuosos. With the pun playing on guitar or (more rarely) bass "chords," it provides a replica in miniature of the narrative that states that women are less likely to be virtuosic at the guitar when and if they ever decide to pick one up, whereas for men, the sky is the limit (Bayton 1993; Clawson 1999).

Another example of difference showed up in the sponsored clothing section. As you play through a chosen instrument, you have the opportunity to unlock clothing, accessories, and instruments from sponsors such as Line 6 for guitars, or Vater Drumsticks for drums, then wear them to pledge your allegiance to your manufacturer of choice. This emulates the rock music industry, where artists will obtain sponsorships from companies that supply them with free gear (see Figure 21) and payment in exchange for exclusive use and advertisement of their brand:

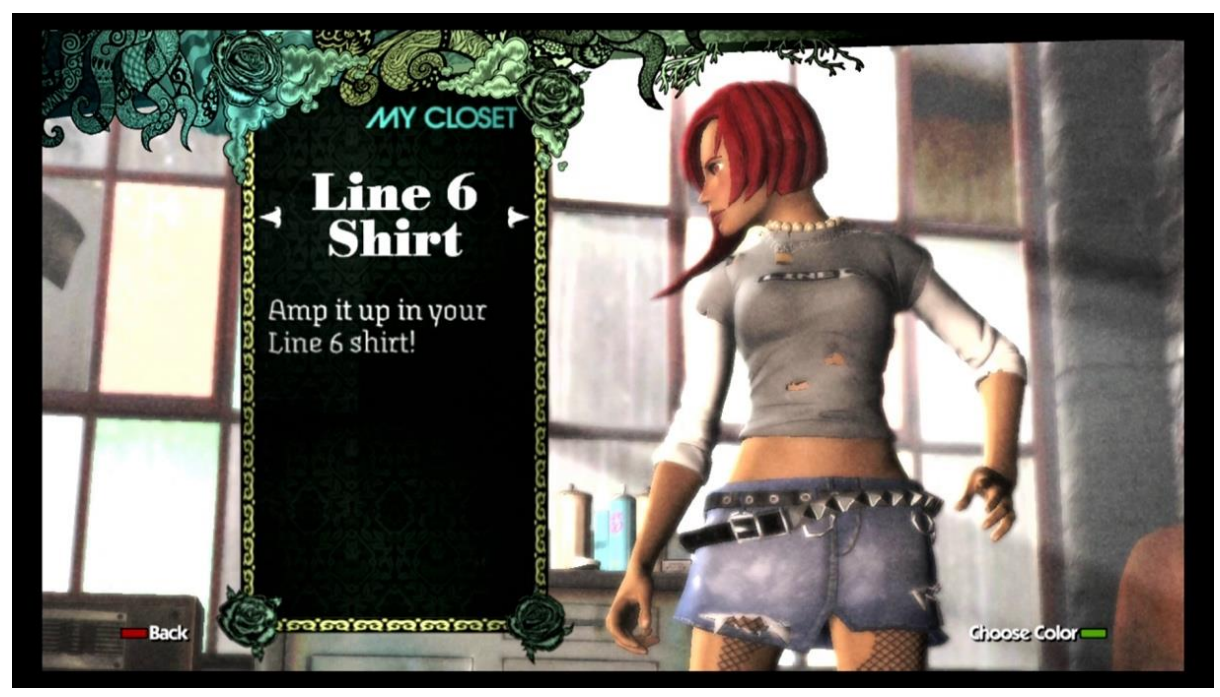

Figure 21: A Line 6 shirt unlocked through successful guitar play. 
The Vater Drumsticks shirts were the only example of sponsored company clothing that had different text attached to the same item:

Men: Vater Drumsticks Tank: Tap out beats with your Vater Drumsticks in this comfortable tank.

Women: Drumsticks Cami: Tap out beats with your Vater Drumsticks in this cute cami.

While the difference between a cami and a tank can be chalked up to differences in fashion terminology (albeit still gendered), it is the difference between "cute" and "comfortable" that gives pause. The implication here is that women are to concern themselves with their attractiveness while wearing this sponsored shirt, whereas men are interested in comfort (perhaps resulting in a better ability to execute the drumming task). In fact, out of the segments of loading screen text that were coded for denoting some reference to attractiveness $(n=37)$, the majority of descriptors (hot, sexy, cute, etc.) were listed as part of women's clothing, makeup, and hair descriptions.

These discrepancies between avatar clothing and makeup descriptions show that, despite attempts at inclusive narrative design, an attempt to add flavor or variance can unwittingly lead to reification of gender norms surrounding musicianship, virtuosity, and desirability.

Playing at Performance

The game's visual design also aids in presenting an interactive simulacra of the rock musician experience. The movement of the characters (both your avatar and non- 
playable characters), the venue options, and the character customization options

(clothing, hairstyles, makeup, accessories, instruments) contribute to a stylized version of Western rock music rooted in allusion and callbacks.

In order to examine the game's visual design more deeply, I engaged in a playthrough of Rock Band with an avatar of my own design. In particular, I wanted to focus on two things: avatar generation and movement, as well as what movements I as a player engaged in throughout the course of play.

I engaged in both Solo Tour mode (which has you play pre-constructed setlists in an effort to earn fans and currency) as well as Solo Quickplay, although I spent most of my play time in Solo Quickplay. In Solo Quickplay, one can play songs individually without the added narrative element. I played as a guitar player with a plastic Fender Stratocaster-like guitar. I found that throughout my endeavor, my surrounding band (a bass player, drummer, and a singer) were randomly generated, although there was less random generation for the singer. The singer's appearance was based on the gender of the singer of whatever song I was performing. This appears to be a move to eliminate any cognitive dissonance between the voice of the actual singer and the appearance of the singer-avatar while playing. This focus on the voice is an interesting one-as discussed later in this study as part of a player survey, voice and the use thereof is a particularly fraught issue due to the complicated linkages between voice and gender identity and performance. Deena Weinstein (2004) asserts, replacing a singer in a band "seems like getting a head transplant. You can imagine a new kidney or a heart-lung implant. But a new head? There have only been a few successful operations (329)." One example of a successful operation was metal band's Judas Priest's replacement of singer Rob Halford 
for a tribute band vocalist that emulated his range and tonality — it was only through perfect simulation that fans were able to accept this change in lineup. In Rock Band, it is possible that a change in visual gender performance may be just as dissonant to players as having a same-gender singer with a widely different vocal quality.

In addition to the loading screen text and avatar movements, I noticed that other loading screens would appear as you further progressed through your makeshift band's career, featuring vignettes chronicling the rise (if you did it right) of your band. These included a fight next to a broken-down van (as seen in Figure 22), your band on the cover a magazine, a shot of a larger tour bus with your band's name on it, etc.

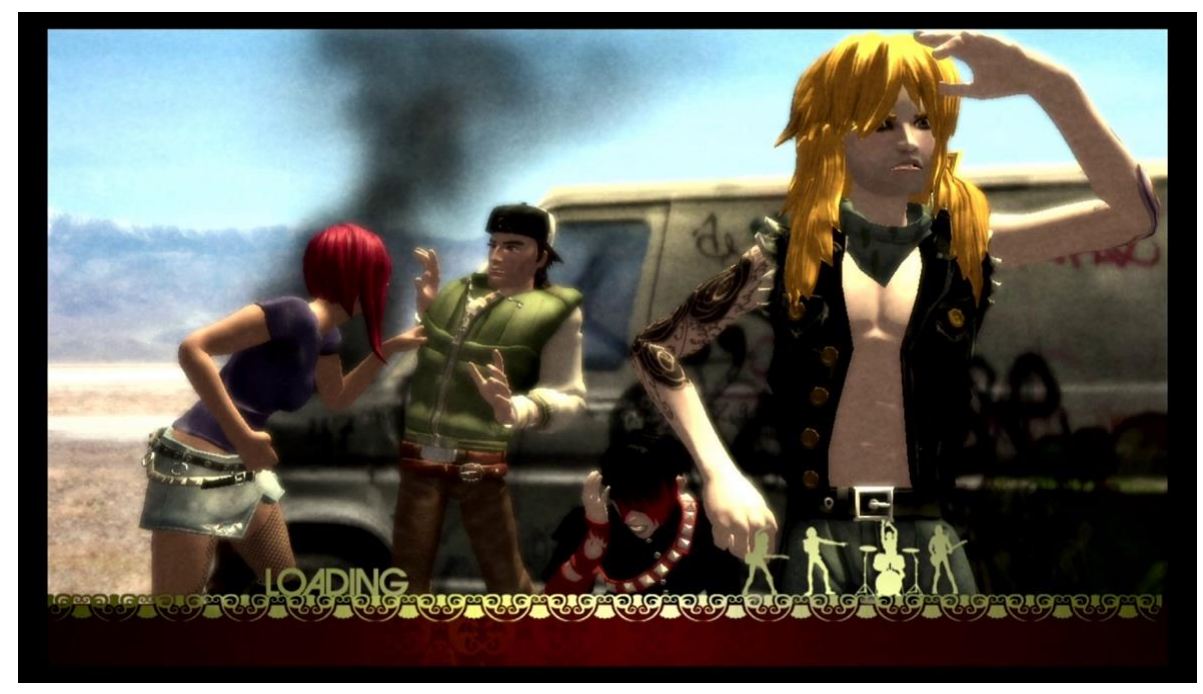

Figure 22: Being on the road is hard. Screenshot from Rock Band.

If you have the cultural capital to recognize these moments in your favorite band's careers, these make fun callbacks. If you are not as familiar with typical rock band trajectory, this serves as a potential lesson on what you might expect if you decide to trade your plastic instrument for a "real" one. 
Another factor that I observed were movements (which I label "rock choreography"), which did not appear to carry the same gender replacement as singers. The rock choreography appeared to be unisex, with changes in choreography defined by musical genre rather than the sex designation of the avatar. In addition to your name and physique, you can select your attitude as part of your initial identity as a "rocker." The attitudes dictate an avatar's facial and body movements on stage and are divided by genre (see Figure 23): rock, punk, metal, and goth.

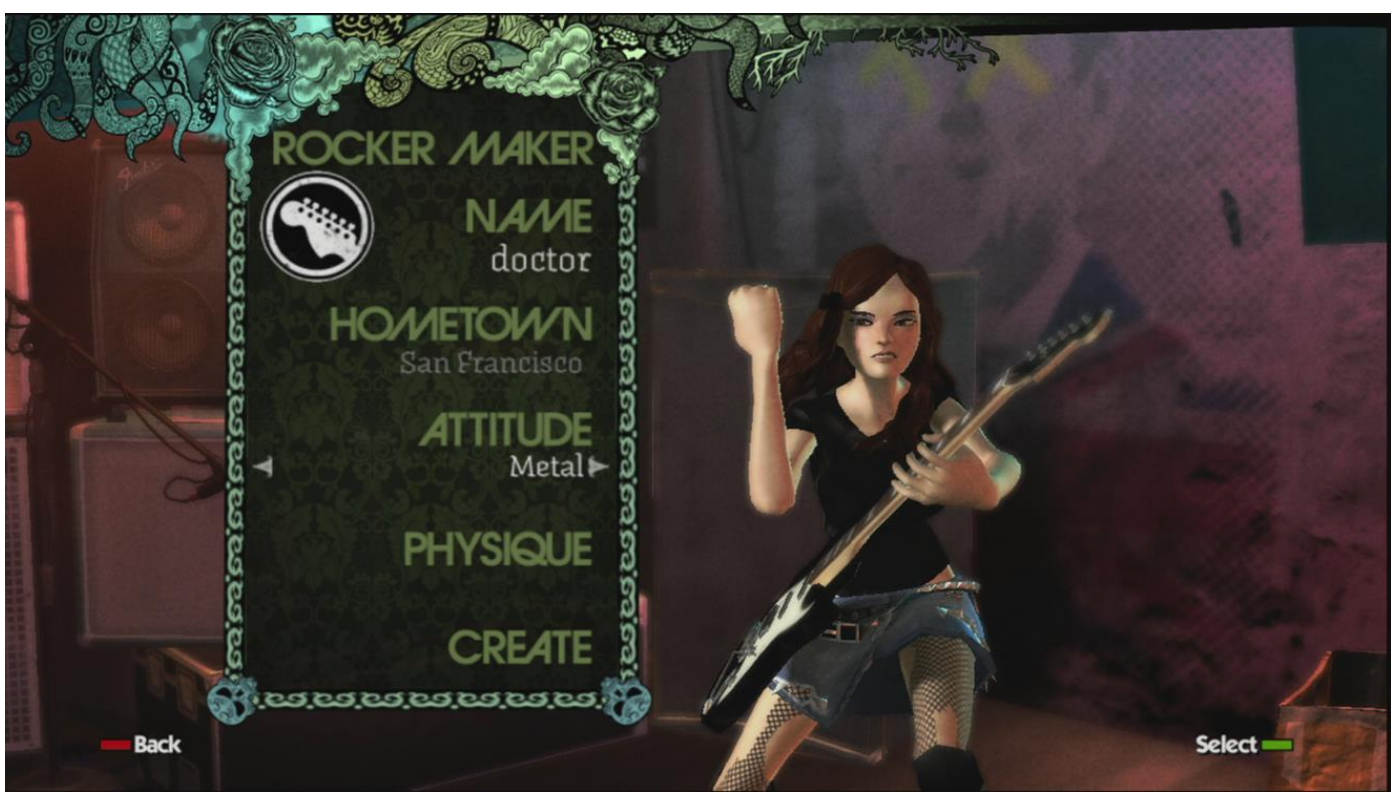

Figure 23: You can select and preview your rocker's attitude, which dictates your actions onstage. Screenshot from Rock Band.

For the rock attitude, the avatar bounces up and down with the beat and punches the air, while the face remains relatively neutral with a hint of a smile. The punk attitude is slightly more aggressive, with a forceful strum of the guitar and a bit of a facial sneer. The metal attitude is the most aggressive of the four, with the avatar kneeling, sneering, and shaking a closed fist. The goth attitude is more flowing and dramatic, with a slow swooping strum of the guitar, swinging the guitar upward, and swaying rather than 
stomping, with eyes slightly closed. The movements are in line with the genre's origins and appeal. For instance, punk is made to be more aggressive as a result of its political leanings - it is sloppier, erratic, anarchic, a response to its more polished pop/hippie/disco counterparts in the 70s, born of disaffection and a Summer of Love that bore sour fruit. Rendering rock movements unisex is something Harmonix emulates in other rhythm games they produce such as Dance Central. In a vacuum, this could show a player absent of other popular music cultural capital an all-inclusive literal and figurative performance of rock music.

\section{Findings}

Initially, I sought to answer the questions of how Rock Band is gendered, how much the game borrows from both gaming and music discourses, and how much of this gendering is hard-coded into the game itself. While I did see some expected results, I did run into some surprises that made me rethink my initial hypotheses.

The answers to the how of Rock Band's gendering as well as what pedigree it claims from gaming and music discourses is found in the same places: set list discrepancies, avatar customizations, and loading screen text. In its faithful replications of music discourses, it ends up carrying sexist baggage along with it, thus gendering the game. Its lack of representation of women in the song list is a play from the existing rock music canon - even as Rock Band includes entries from pop music, which can be friendlier to women, primarily as vocalists. It's a faithful replication of a world where, in a 1988 Rock n' Roll Hall of Fame ceremony (where canon is established and added upon), the only two women on stage were Mary Wilson, a pop vocalist, and Yoko Ono, a 
symbol of wives and girlfriends breaking up all-male bands, accepting an award on her late husband's behalf (Garr 1992). While there are opportunities to subvert these gendered pop-rock canonical norms by oversampling women, the $16 \%$ representation is a hard-coding of gender roles in rock, in video game form.

The gendered hard-coding also takes the form of the avatar make-up and clothing customization options, and it is here where we see slight glimmers of subversion, and a potential answer to the third research question, of where subversive opportunities lie. It becomes a perfect cover version of rock music discourses to include opportunities for androgynous performance through makeup, hair, and clothing. It becomes subversive when you consider that rock music and a lot of its subgenres are active responses to mainstream social norms of conformity and tradition, and the game's options may provide a way for players to experiment, play, and construct more authentic gender presentations for themselves via a system that was merely created to make sure you could be a proper glam or punk rocker.

Another potentially fraught category is the category of loading screen text, where the text, unchangeable by the player, hard-codes messages about the authentic-fantasy rock star experience. The most salient example was the only concretely-gendered pronoun use in personnel loading screen tips, referring to the "Sound Guy." While I was looking for answers having to do with hard-coding of gender for the musicians themselves, it was this bit of text that reminded me that representation is lacking in other areas of rock music culture and technology, and it further limits the roles women could potentially play within it. 
The most surprising of all was in the interviews with the developers, where I saw the most shining example of subversive opportunities within music games-subversion from the inside, in the form of intentional inclusive design. By having more women in the games writing rooms that are conscious of bias, it appears to have played a role in creating what Fullerton et. al. (2008) call "a virtuous cycle," where games are created that are friendlier to women and thus could draw more women into game creation, thus creating a more inclusive slate of games for people of all genders to play (175). This example is seen in the Harmonix writer who, during the writing process for a game, asserted that an achievement title had a particularly gendered connotation that made an assumption of a male player-character. It is possible that, had they not expressed themselves, the achievement may have gone unchallenged at the risk of alienating a segment of players.

I hope that these findings speak to those with a vested interest in not only music games, but video games and other forms of popular culture. While it is valuable to examine what may be considered more "obvious" forms of popular culture (movies, television, music) or more "obvious" game subject (ultraviolent and ultrasexualized), I find it necessary to apply the same critical lens to all genres of video games-especially those that might be available for younger audiences than games typically rated $\mathrm{M}$ for Mature.

Along with prescriptions for academics in the field, I hope that these findings find their way to the industry at large, particularly the findings of how having diversity in the writers' room (and the design room, art room, etc.) can lead to more potentially intentionally inclusive design. In particular, the inclusion of more women in the music 
game design process can mean a virtuous cycle for both women who play games and women who play instruments, where they can each see themselves represented and potentially become inspired to pick up a drumstick, a controller, or even some coding software. 


\section{ROCKSMITH: THE NEXT STEP...BUT IS IT PROGRESS?}

Rocksmith represents a convenient evolution of the rock-based music game genre. While this game may not have been the first to use a "real" guitar with six strings, it was one with a major publisher (and thus, marketing spend) behind it. It was also convenient in that, just as I was inspired to take on the task of adding music video games to my feminist research interests, I became a Frag Doll Cadette/marketing intern for Rocksmith's publisher Ubisoft and was assigned to promote this particular game in 2011. I had already begun the process of using my newfound access to trade shows to begin contact with the developers of Rock Band, Harmonix, and found Rocksmith both an interesting add-on to the analysis as well as convenient due to my proximity with the development team.

Initially, I set out to examine Rocksmith the same way I did Rock Band, with a focus on research questions 1 and 2, focusing on the ways in which Rocksmith is gendered, what tropes it carries over from music and gaming discourses, and the ways in which gender is hard-coded into the game. I expected to find a dire situation for the representation of women musicians with the set list, although I was not sure what other opportunities for hard-coding of gender I would find in a game that did not feature a customizable player character. In order to find the answers to those questions, I employed content analysis of the song's set list, and was spurred to seek other forms of analysis by the lack of player character, my unique position as both researcher and marketing asset for the game, and, eventually, a deeper dive into the game's design inspired by the developers themselves. 


\section{Ubisoft \& Rocksmith}

Ubisoft was founded in 1986 in part by Yves Guillemot, and boasts the " $2^{\text {nd }}$ largest in-house development staff in the world with 29 studios in 19 countries," as well as being among the top five publishers in the world per their website (ubisoftgroup.com). Their North American headquarters, however, is the studio that holds the majority of production and marketing efforts for Rocksmith and its successor Rocksmith 2014.

Rocksmith can be considered a next stage of evolution for the rock music video game. Rocksmith merges the video game with a musical teaching tool, using the familiar Rock Band noteway interface to visualize songs that can be learned via any real electric guitar or electric bass. Previous attempts at bridging the gap between simulated plastic peripherals and authentic instruments created proprietary hybrids — guitar with strings that could only be used within the game's interface or through electronic (MIDI) intermediaries (Rock Band's pro guitar, Power Gig: Rise of the Six String). For Rocksmith, any guitar that can be plugged into an amplifier via a 1/4" cable is eligible for use with the game through the use of their proprietary $1 / 4$ " cable to USB adapter which plugs into your console of choice or PC. As these types of guitars are the most widely available in your average guitar stores and would be the ones people would purchase for public performance, the game's technology makes it (and, ultimately, instrument knowledge) more accessible to people.

The first installment of Rocksmith was released in October 2011 and allowed you to plug in a real guitar. A 2012 expansion added bass to equation, using the same cable to allow you to learn how to play bass as well. In 2013, successor Rocksmith 2014 brought 
an overhauled user interface as well as session mode, which lets you play along with a “session band” of your own design. In October of 2016, Rocksmith 2014 Remastered represented a reworked version of Rocksmith 2014 which included improvements to song sorting, adjustable difficulty levels, and the ability to do away with the cable and play an acoustic guitar using a USB microphone to pick up the sound. The selection of Rocksmith as a case study made sense due to its release by a major developer and publisher versus competitors such as BandFuse or Gig: Rise of the Six String. With the strength of a major publisher and marketing team behind it, Rocksmith is the more visible title and the more likely one to end up in people's homes, potentially shaping or reshaping people's guitar- and bass-playing experiences.

\section{The Rocksmith-smiths: A Look at Creation and Design}

I had the chance to interview three developers at and near the Ubisoft San Francisco headquarters in spring of 2013, during the Game Developers Conference (GDC). While one developer provided insight in to Rocksmith's origins and commitments to authenticity, the other two provided a look into the development of key design features that changed drastically between the first iteration of Rocksmith and its sequel, Rocksmith 2014.

The first meeting took place outdoors (leading to its own audio gathering challenges) in a park close to the GDC expo halls. The audio designer I spoke with was involved with Rocksmith since before it was known as Rocksmith, since before the technology was obtained by Ubisoft. At its inception, it was known as a game called 
Guitar Rising, and it was demoed during a 2008 GDC in which it received high praise for its note-detection technology involving the use of a real guitar.

As part of the conversation, he talked to me about an as-yet-unannounced feature that was part of Rocksmith 2014, Session Mode. Session Mode gives a person playing the game on guitar or bass the ability to "jam" or play along with a band of one's own design made up of 77 instruments (including different kinds of guitars, basses, drums, keyboards, percussion, and even a kazoo). As soon as one starts playing the guitar or bass, the "band" kicks in, and they follow along with the player's speed and intensity. During that period, the game feeds the player images of the guitar or bass neck and pointing out appropriate notes to play depending on the key they're in.

The creation of these 77 instruments, according to the audio designer, was an effort to create a band that "listened to you," a quote turned marketing point that I would later experience once the game was announced at that year's Electronic Entertainment Expo (E3) later that year. In creating that band, there was a marked focus on authenticity, beginning with using working musicians to record the different instruments rather than relying on store-bought samples:

...the guy that I had actually wrote all of the riffs for the keyboards- - like he was a piano player and a jazz player straight up and down. That's all he did. He wrote all of the riffs for the keyboards, so he knew (Developer 1, Ubisoft).

As part of the recording process, he also made sure to further the commitment to making the band feel more "human:" 
Still using piano as an example, a lot of the riffs that went in at first were like "doodilidoo doodilidoo" And I'd listen to it and be like damn, that's fucking sick, but it doesn't sound like a person because nobody can play that, and if they can arguably those people sound like robots. ...we actually had to make sure that the actual instruments sounded very straight forward, as if you were playing with them, like real people. On a lot of the instruments we actually went and put mistakes [in] because otherwise they [didn't sound] enough like real people (Developer 1, Ubisoft).

Back at the Ubisoft San Francisco headquarters, I met with a lead notetracker and a game designer. As lead notetracker, he was responsible for transcribing songs that would go into the game in order to translate them into the game's vocabulary. He also worked with an artist to contribute to the game's design, specifically some of the visual feedback you receive as part of the game when you're playing. The notetracker has synesthesia, so he sees specific colors when he hears music or sees music notation:

There's...so many songs where it's an 'E' and it'll be a blueish hue. 'E' and 'A' are both kind of blues. 'C' and 'D' are greens. And when you get to the flat keys, so if you're playing Weezer, you'll see everything is gold because flat is gold. These aren't the colors I see-This is what the artist and I came up with as far as coming up with the stuff. There's even secondary colors which are following kind of the harmonic progression (Developer 2, Ubisoft).

This, he explains, creates a secondary learning experience where players begin to make associations between notes and colors in the hopes of training their ears better. In the end, 
not only is Rocksmith a game, but it is a teaching tool. In fact, future marketing materials would suggest that Rocksmith was "the fastest way to learn guitar," adding to its authenticity factor. Where Rock Band was made to simulate the experience of playing guitar with the possibility of learning once you got separate "pro" hardware, Rocksmith's mission from the outset was to close the gap between game-player and guitar-player through learning with any "real" guitar or bass.

In addition to the lead notetracker, I interviewed a game designer who immediately opened up once he learned about my subject of study and told me about changes in the game's design between Rocksmith and Rocksmith 2014 that were specifically gender-based:

In 2011, when we got our analytics back, we saw it was like $99.7 \%$ male or something like that. I don't know the exact number, but close to that. Our player base was $99.7 \%$ male... of people who did the survey and we got like 5,000 people to do the survey, so it was like a pretty good chunk (Developer 3, Ubisoft).

While I make an argument later in this chapter that the heavily skewed player demographics were aided by marketing, the game designer laid the blame squarely on the shoulders of the game's design, from gameplay elements to instructional videos:

...if you remember Rocksmith 2011 had this very musky feel to it and it was very like whiskey, dark brown... and that was the direction we went and the direction we were even [going] when we were even making these videos... I forgot the name... the "greatest man in the world" type thing (Developer 3, Ubisoft). 
"The Greatest Man in the World" was a series of ads by beer company Dos Equis detailing the fantastical adventures of a leathered, husky-voiced older Lothario. It appears that Rocksmith initially wanted to appeal to the same aspirational demographic of people who would want to achieve legend and drip with cool as a result of learning the guitar. Indeed, the design of Rocksmith bears this out, as the game's opening (see Figure 24) stars a headless man in a leather jacket playing through the games theme song in a darkened room with several guitars:

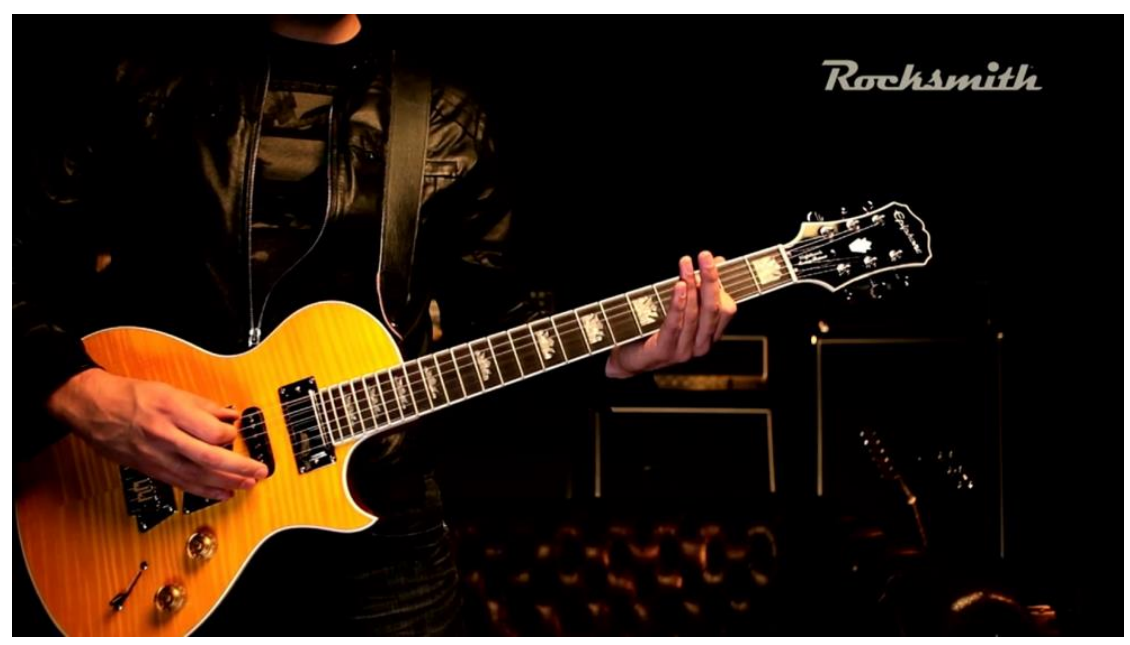

Figure 24: The 2011 Rocksmith intro, featuring a headless leather-clad guitarist in a darkened room. Screenshot from Rocksmith.

The theme carries through the game's menus (see Figure 25), which are dark with white and yellow-orange text. The original Rocksmith carried you through a journey where you earned points by doing well on songs, which unlocked different venues to perform in and customizations for your guitar (including cosmetics, pedals, and amplifiers to customize your guitar's sound): 


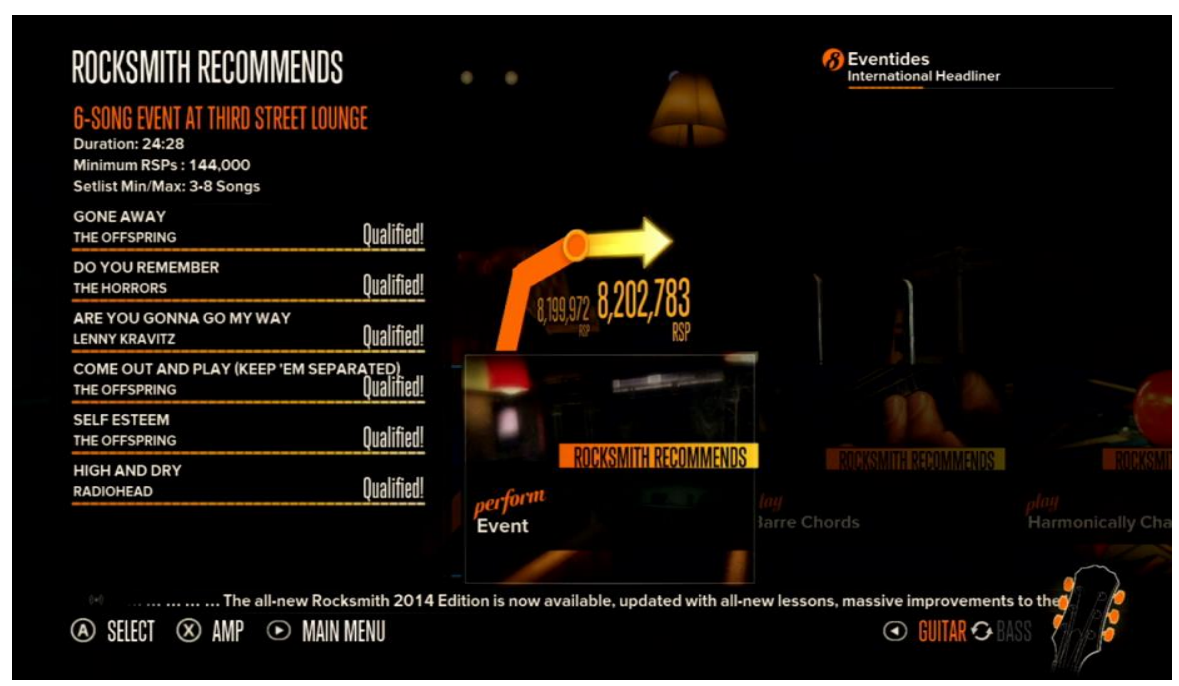

Figure 25: The Rocksmith main menu, which outlines your point progress and labels you based on your experience. I reached "International Headliner" status. Screenshot from Rocksmith.

The theme carries all the way through to the instructional videos (see Figure 26), as stated by the game designer. The headless man returns as instructor, showing how to wear your guitar:

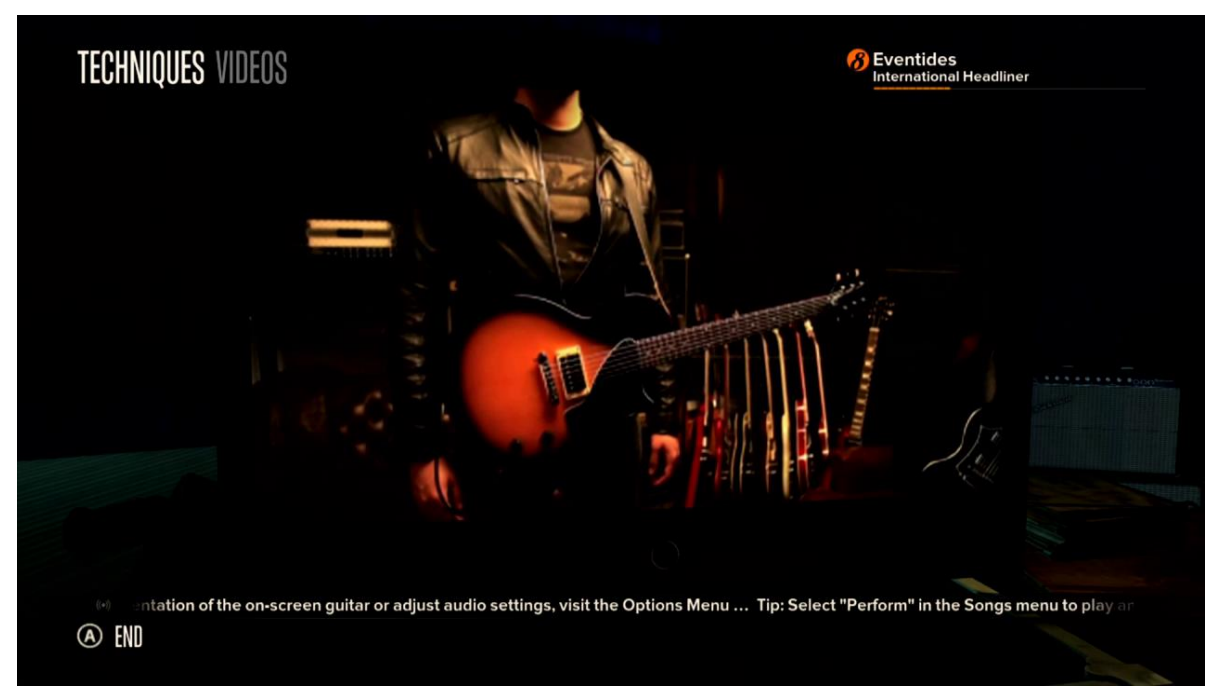

Figure 26: The headless leather-clad instructor, in darkened room. Screenshot from Rocksmith. 
As a response, the team decided to change the overall visual design in the hopes of being more inclusive:

...So, in 2014 we're like okay, we're gonna change the whole direction: we're gonna brighten it up, we're going to make it light--- we don't want girls feeling, "this is something just for my dad," and so we totally changed it... You could tell the difference in the videos. The videos, the previous ones: the very dark room and in Rocksmith 2014 we decided to make it a very bright loft. It's studio space, it's bright, it's vibrant colors...(Developer 3, Ubisoft).

The design choices even extended to casting for the in-game guitar and bass lessons, where they made sure to hire both a woman and a man for the guitar parts but ended up with only one bassist (a man) due to budget constraints. This reaction also came as a result of their demographic survey revealing an older population than they had initially intended:

What ended up happening was a lot of our player base happened to be people who... went through their careers and started families and their guitar is left in their garage ....and then Rocksmith comes out in their late 30s or their 40s and they're like, "you know what? I have time now" and end up picking up the guitar again. ...Those type of people... want to introduce music to their kids... Usually the type of girls that would get into the game were people whose parents were playing the game so we wanted to make sure that it was more accessible (Developer 3, Ubisoft). 
In fact, their original goal was to reach the Guitar Hero-playing audience who wanted to transition to playing an instrument. This emphasis on catering to a "gamer" demographic with rock-star aspirations based on entelechial, score-based, perfection-based motivation (Soukup 2007) led to a playerbase that mostly resembled the "gamer" stereotype, albeit skewed older. For Rocksmith 2014, however, they designed and marketed the game to "anyone who wants to learn to play guitar" in an effort to make it more accessible.

Rocksmith 2014's design is a departure from their previous effort. The start screen that once had a headless leathered guitar player cycling through different guitars is replaced by game developer logos on pedals, game technology logos, and a start screen on a white background (see Figure 27):

\section{Rocksenith son somono REMASTERED}

Begin

Figure 27: The Rocksmith 2014 start screen. The "Remastered" comes from a reworking of the game that released in Fall 2016. Screenshot from Rocksmith 2014.

The menu screen is brighter, with the background being an open, airy bright loft space (see Figure 28) instead of the previous dark aesthetic: 


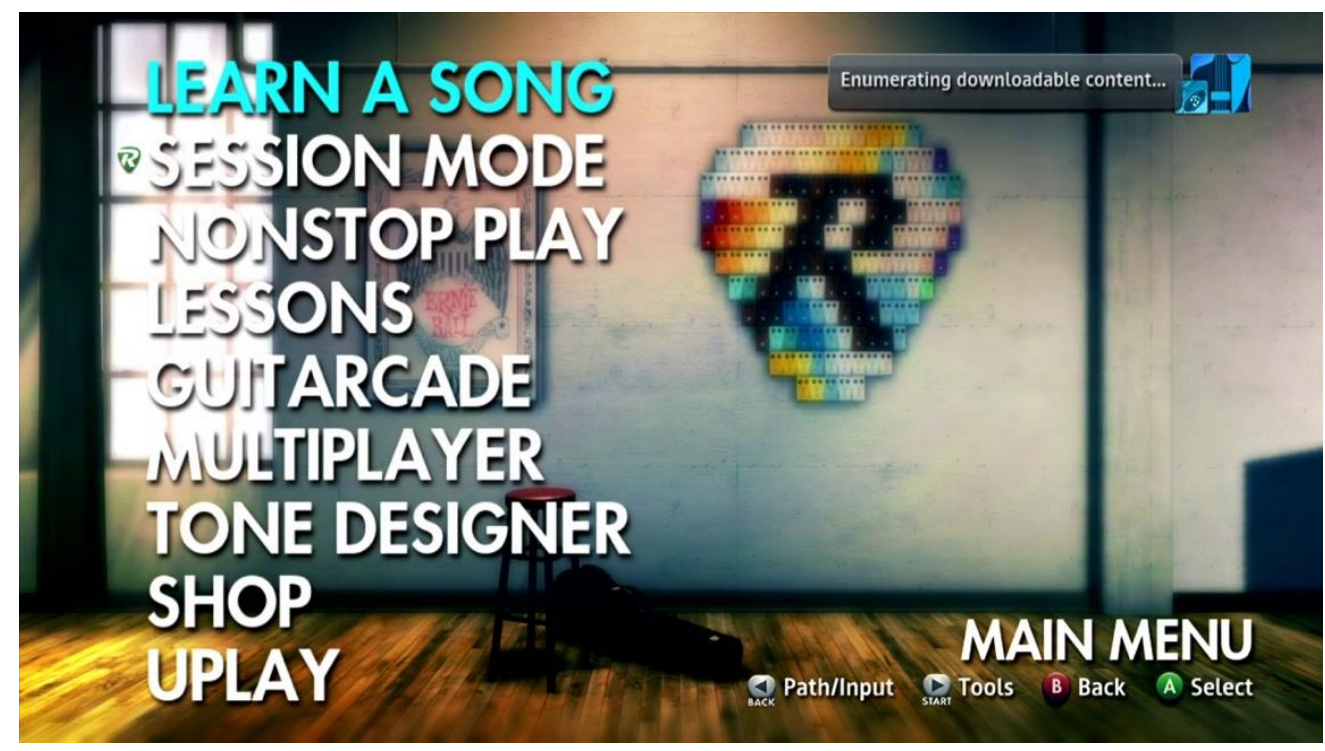

Figure 28: The Rocksmith 2014 main menu. Light coming through the window plus a brightly-colored wall accent made of guitar pedals. Screenshot from Rocksmith 2014.

The lessons see a similar upgrade. In the menus, you see representation from the male and female guitar player (Figures 29 and 30), and the lesson videos are just as bright and colorful as the rest of the design. The clothing options for the male instructor is more reminiscent of an "indie" style, with a bright plaid shirt and skinny tie versus the dark jeans and leather jacket of the original instructor. Instead, the female instructor is clad in the "harder" ensemble of the two: 


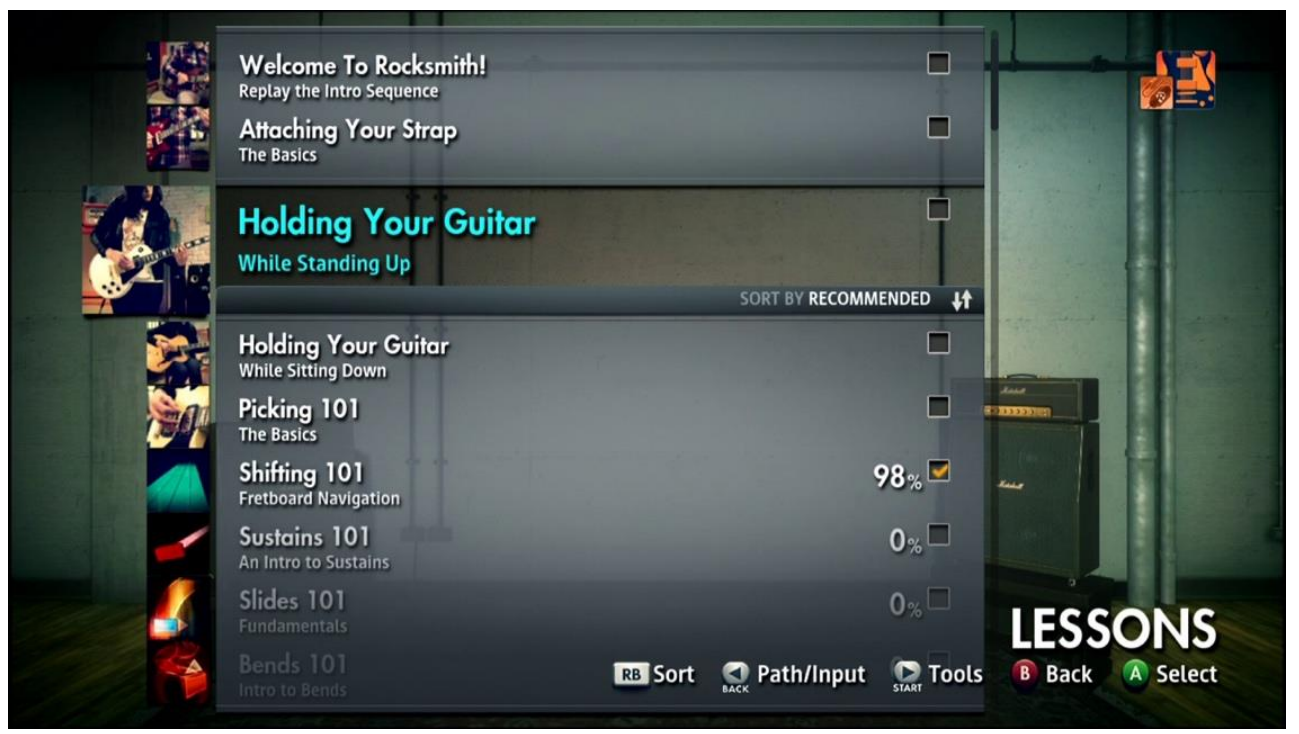

Figure 29: The new lessons menu, featuring a woman instructor as the thumbnail for the guitar-holding lesson. Screenshot from Rocksmith 2014.

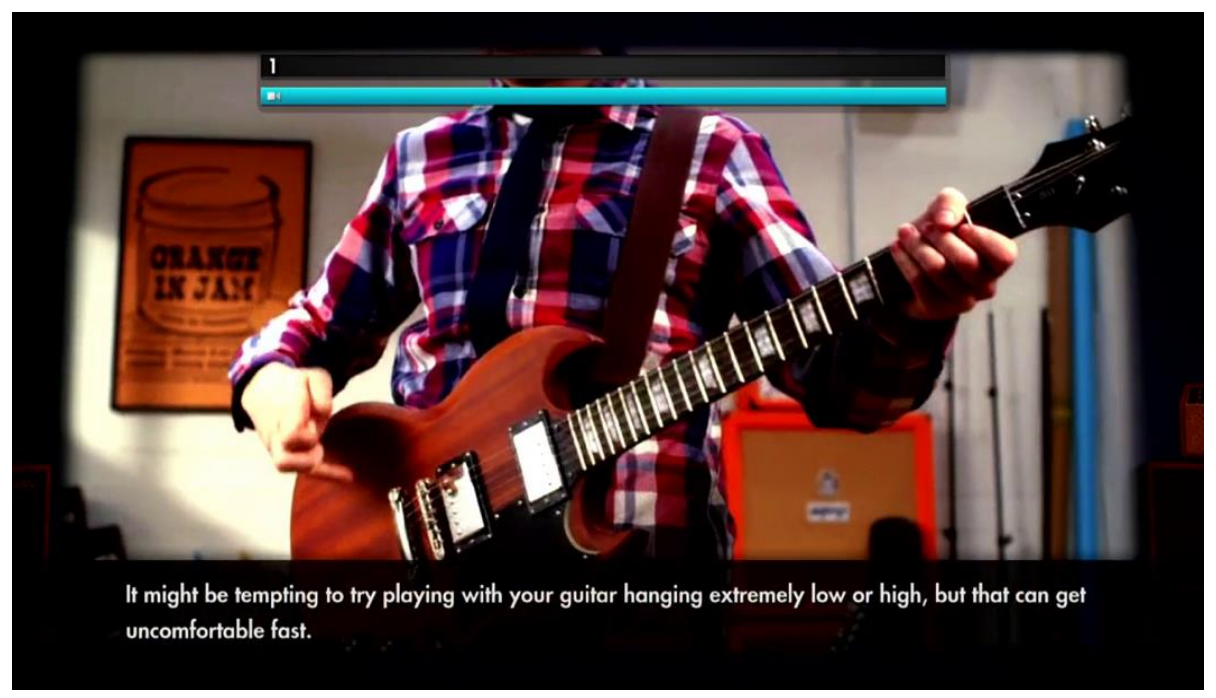

Figure 30: Lessons occur in a brighter room, with the instructor even wearing brighter colors. Screenshot from Rocksmith 2014.

Another difference between the 2011 and 2014 versions is the way performances are presented. Each game has an option to practice songs as well as perform in front of a crowd. For the 2011 version, the practice performance took place in the same living 
room, while the "real" scored performance took place at different venues with a simulated audience (which you unlocked throughout the course of the game). Also of note is how the numerical score appears on the upper right of the user interface (Figures 31 and 32), updating itself as you progress through the song:

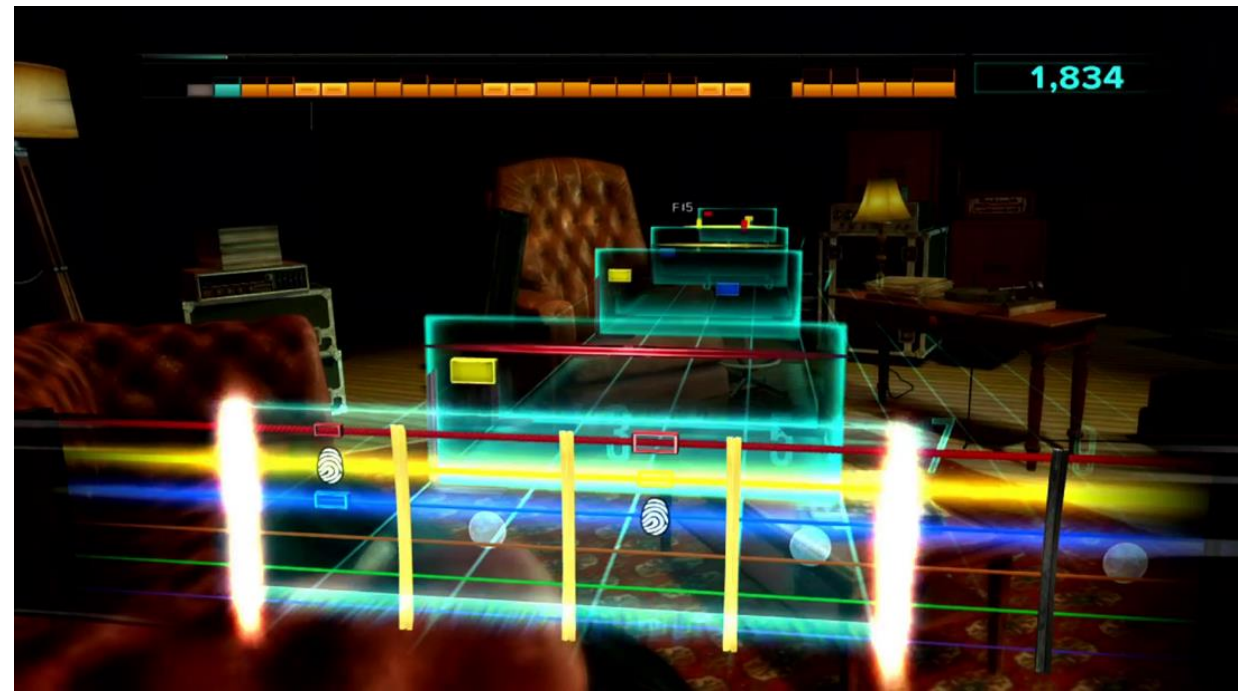

Figure 31: In the 2011 version, you practice in the same darkened room as the intro. Screenshot from Rocksmith.

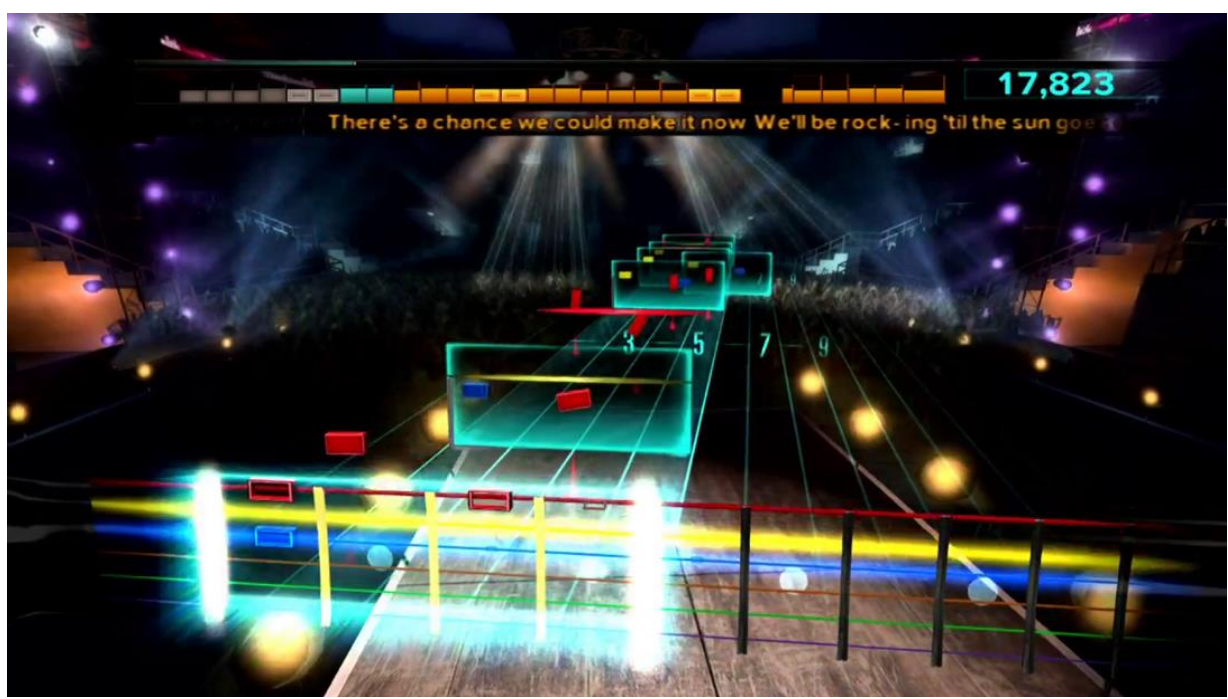

Figure 32: Performing in front of a crowd. Screenshot from Rocksmith. 
In the 2014 version, when you are playing a song, you begin performing in a neutral room (see Figure 33), with the audience revealing itself to you the further you progress through the song and the better you perform:

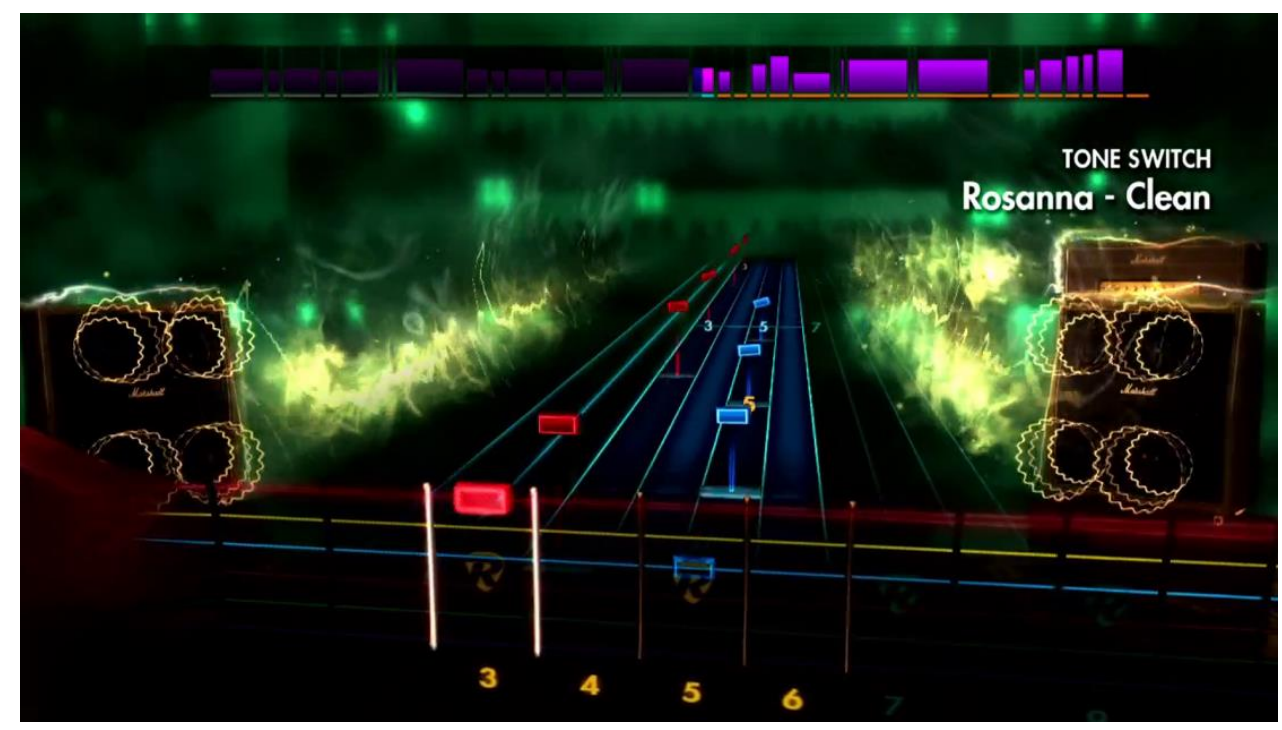

Figure 33: The fog clears to reveal a cheering audience-if you do well enough. Screenshot from Rocksmith 2014.

I argue that the slow reveal of the audience in 2014 renders the game more accessible versus its 2011 counterpart, where their cheers (or lack thereof) are present from the beginning. Those familiar with Guitar Hero and Rock Band playing the 2011 version may already expect the crowd to turn on them if they miss a note or two in Rocksmith, which may not be the friendliest form of learning. In addition, the lack of numerical score feedback on the upper right hand of the user interface makes it so that one focuses on the feedback given on the noteway as the song progresses, making adjustments to play. The focus here, then, is on improving your guitar playing rather than an arbitrary score.

The actual scoring method is also different between the 2011 version and the 2014 version of the game. In 2011 (see Figure 34), you were awarded Rocksmith points 
based on skill, and those points counted towards leveling up, unlocking new venues, and towards guitar customizations. While the guitar customizations still exist in 2014 (Figure $35)$, the scoring system is based on pure percentages of accuracy:

SONG RESUITS REPLAY

I BELIEVE IN A THNING CALLEO LOVE

COMBO

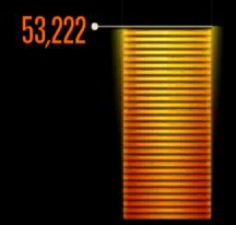

ARTIST

ALBUM

The Darkness

LONGEST STREAK

NOTE ACCURACY

Believe in a Thing Called L...

(A) NEXT
(8) Eventides

International Headliner

Figure 34: Your success in Rocksmith determined by points. Screenshot from Rocksmith.

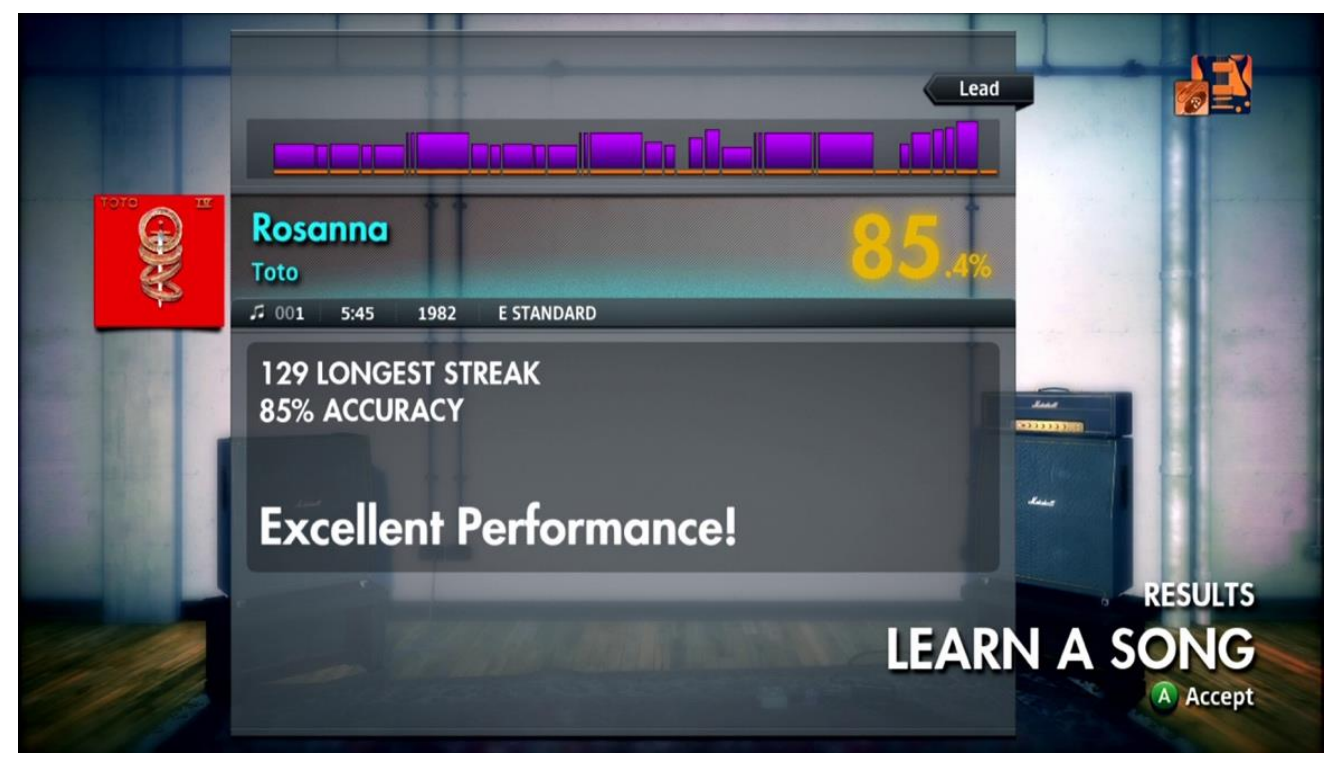

Figure 35: Rocksmith 2014's scoring, based on percentage. Screenshot from Rocksmith 2014. 
By moving from a purely score-based system that leans on prior experience with games such as Guitar Hero and Rock Band to a pure percentage-based system, Rocksmith 2014 becomes more accessible as a teaching tool that happens to be a game rather than a game that happens to rely on guitar.

\section{A Note on Avatars}

One of the similarities between Rocksmith 2011 and 2014, and another key difference between this franchise and the Rock Band franchise, is the lack of customizable avatar. While the player gets a first-person view of an audience as if they were on stage, the player character is never rendered on screen. The player character, then, is the player themselves, hitting home the goal of Rocksmith to teach instrument skills and convert the player into the rock star rather than a facsimile of one.

Aside from the lack of customizable avatar for the player, the instructors in both Rocksmith games are shown from the neck down, focusing attention on the guitar or bass. The audio comes in the form of a voiceover, and the same voice (a person the community has affectionately dubbed "Rocksmith Guy") narrates the lessons regardless of the gender of the instructor. Initially, I thought the choice had to do with keeping the focus on the instruments and hands, in order for the player to see techniques better. According to the designer, as with many game design decisions, the choice of having headless instructors came down to budget and logistics. In order for games to be sold in territories worldwide, they have to be "localized." The localization process goes beyond mere word-for-word translation, but also includes mindful translation of concepts relative 
to a territory's sociocultural attitudes. The localization process is costly and timeconsuming, leading to the decision:

The first primary reason is we wanted to make sure this was localized in many different languages, so it would be a bunch of translations... we had to spend the resources to find an actor that could also speak [the language], that would make it that much more difficult, and it means it would have to find a studio space where it's very important to record sound at the same time. We decided that it would be cheaper... a lot of decisions in games is what comes out cheaper, right? (Developer 3, Ubisoft).

What I initially thought was a decision rife with intent and commentary on the nature of avatar identification and the idea that you as player are the star of this Rocksmith journey, it all came down to simple math. It was through this interview that I recognized how many decisions in games could be made because of budgetary concerns and end up having authorial intent projected onto them. However, I would argue that, absent of intent, a game can be read the way its read, and it is no less a "real" reading. I would also argue that, regardless of intent, and regardless of whether the instructors have heads or not, it is still valid to question the casting choices for the instructors and the reasoning behind the choice, considering it's such a departure from previous games in the genre.

With the success of Rocksmith in 2011, the development of the 2014 version was given an increased budget. While they could have made another design change and added either avatars or a more interactive personality behind the lessons (and given the instructors heads), they opted not to: 
...if we take the head out, it's all emphasis on guitar, and all emphasis on your hands... we almost tried to remove the personality—well, we didn't want to remove personality, we wanted it so that the personality of the person that you could perceive that person to be doesn't detract you from learning and we felt that if there was a lot of face, that could potentially distract you... you could just get turned off by someone's look and so we didn't want that to conflict with your ability to learn (Developer 3, Ubisoft).

The fact remains, however, that the head is not the only source of gender performance, and in the casting of the new instructors, men still outnumber women 2:1 (quite literally).

\section{The Rocksmith Set List}

Rocksmith as a franchise reached a total of 1,000 songs in spring 2017. They continue to release downloadable content on a weekly basis with at least three songs per downloadable pack. The packs are either variety packs, themed packs based on subgenre, or packs featuring an artist. In the same vein as the Rock Band study in the previous chapter, I conducted an analysis of the first 1,000 songs in the Rocksmith library. Despite conducting the statistical analysis a few weeks after the 1,000-song milestone (which would have put the population at over 1,020 or similar), I found it more convenient to focus on the first 1,000 songs. In addition to the number being exactly half the Rock Band population, the Rocksmith developers publicized the 1,000-song milestone with giveaways of a "Golden Ticket," a code that granted the winner the first 1,000 songs. As it was a significant number that was both publicized and accessible to a certain percentage of the playerbase (due to the giveaways), I thought it a fitting 
population to examine. Similar to the previous Rock Band analysis, I coded for whether there is a woman in the band at all ("Female Presence") as well as whether they appear as guitarists, bassists, drummers, or vocalists.

Particular attention was paid to the number of guitarists and bassists, as not only are these signature instruments in rock music, but these are the instruments available to learn as part of the Rocksmith franchise. While players have the opportunity to plug in a USB microphone and sing along, their performance is not judged at all, and there are no vocal lessons in the game. Song difficulty is based on the percentage of notes hit (which is more exact when songs are translated note for note on guitar and bass). Therefore, the analysis takes the form of descriptive statistics (see Figure 36) rather than what was used for the Rock Band analysis.

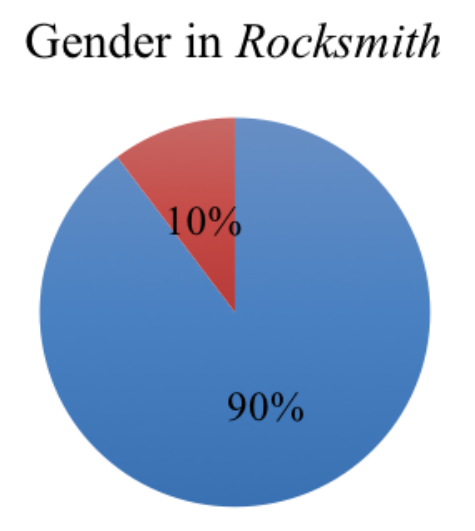

- All Men - Any Woman

Figure 36: Gender makeup of Rocksmith artists. Image rendered in Excel.

The game heavily skews towards musicians featuring all men, with almost $90 \%$ of the songs $(n=897)$ devoid of major participation from a woman in the four major rock music 
instrument groups (vocals, guitar, bass, and drums). For comparison, women in Rock Band represented $16 \%$ of that game's sample. An argument can be made that Rocksmith is more limited in genre scope, not including as many mainstream pop songs (which is where women vocalists thrive in American popular music) as Rock Band does, leading to Rock Band's small increase in women being featured.

When women do appear in bands, their distribution (see Figure 37) is similar to Rock Band's in that woman are more likely to be vocalists than instrumentalists:

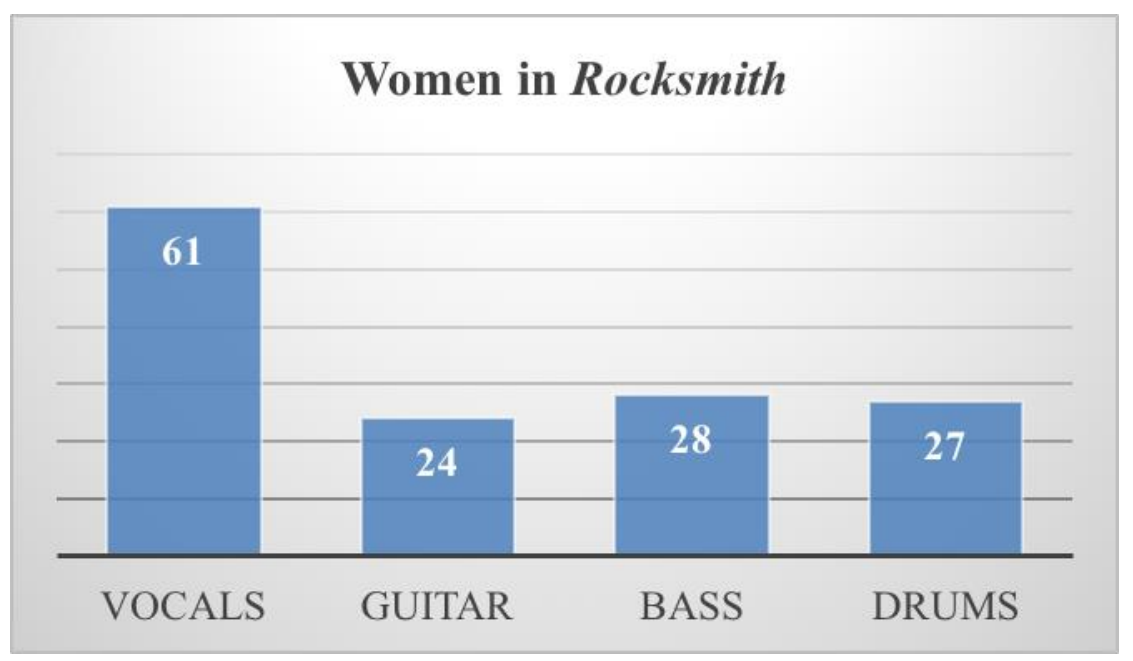

Figure 37: Breakdown of women in Rocksmith. Image rendered in Excel.

As seen above, women are, by a small margin, still more likely to be see on the bass or drums than on the signature instrument of rock music, the electric guitar. For Rocksmith, this is a more egregious slight to representation, as the game only emphasizes learning songs on guitar and bass. One can plug in a microphone and sing along, but there are no lessons nor gameplay segments that feature vocals—even less so for drums. 
This doesn't mean that attempts aren't made, however: The game released a "Women Who Rock" pack of songs coinciding with International Women's Day in March 2016:

Cherry Bomb by The Runaways

Take It Off by The Donnas

Heaven Knows by The Pretty Reckless

I'm So Sick by Flyleaf

Celebrity Skin by Hole

The pack spans pop rock, punk, metal, and post-grunge as well as several decades. To their credit, all but one of the songs in this pack feature women instrumentalists (The Donnas and Runaways are all-female bands and Hole and The Pretty Reckless feature lead singers who play rhythm guitar), but it's a drop in the bucket compared to the wider discography. The game sought to make improvements in the user interface, marketing, and overall attitude of the game to make it more inclusive and approachable—but, Women Who Rock pack aside, it seems the set list can be seen as a major missed opportunity to do so. 
Becoming a Guitar Player...Through Swag

“Swag," or marketing giveaways, are a typical part of a game's overall marketing strategy. At video game conventions, it is not uncommon to see t-shirts, posters, or figurines as common pieces of swag as incentives to pre-order or play the game. Giving out pieces of fashion is a common way to gain promotion for a game, turning your playerbase into walking billboards. Rocksmith, on the other hand, doubles down on the authenticity factor by opting for functional swag that directly correlates with a guitar player experience.

At both video game tradeshows and music festivals, booth presence was marked by guitar straps, guitar picks, and sometimes actual guitars for major giveaways. During the Rocksmith weekly developer livestreams, which occur every Thursday on twitch.tv/rocksmithgame, giveaways include the downloadable content songs of that week as well as a care package (see Figure 38) consisting of a guitar strap, packs of strings, picks, and "Wonder Wipes," which are used to clean dirt and the accumulated oils from your hands off of your guitar or bass. 


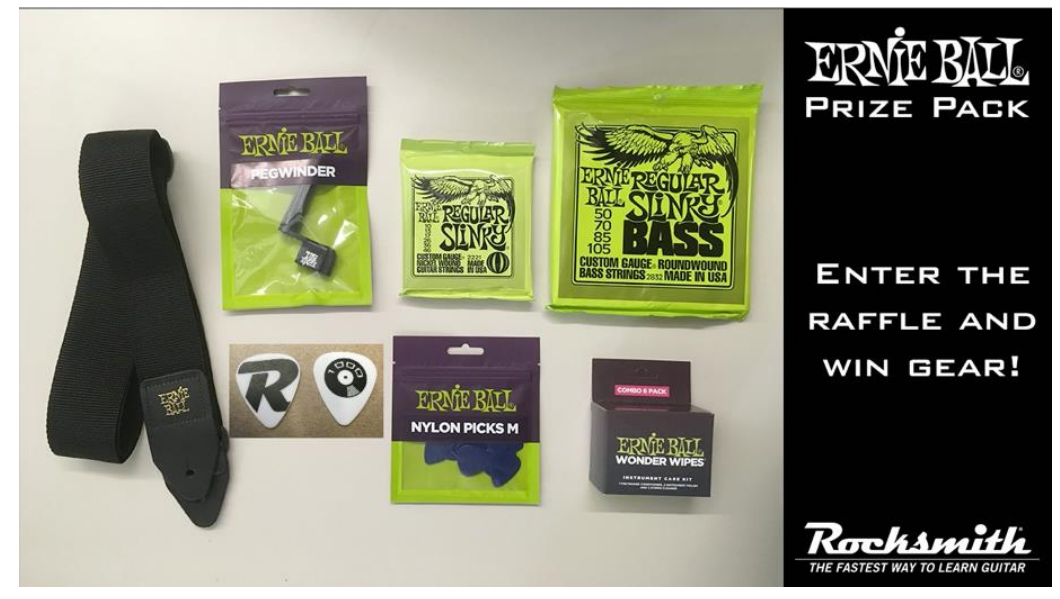

Figure 38: A common Rocksmith giveaway on their livestream. Screenshot from twitch.tv/rocksmithgame

Using guitar-specific swag serves several purposes. By having new guitar gear courtesy of a video game company, it can encourage the player to spend more time in the game and build game/brand loyalty. By extension, the use of guitar gear reinforces that the player is a guitarist or bassist, rather than a simulation of one represented by a stylized avatar. In this case, the player is the main character of Rocksmith and continues to be so through the accumulation of authentic guitar ingredients.

\section{For Those About to Rock, Check Out Our Booth}

As a researcher, my first encounter with Rocksmith was at the Penny Arcade Expo (PAX) in Seattle, Washington in the fall of 2011 (see Figure 39). I was selected, as part of the Frag Doll Cadette team, to work at the booth helping attendees preorder Rocksmith and managing the line for playing the game. I requested to work for this game partly for participation purposes and partly because I was familiar with the guitar and wanted to excel in the hopes of becoming a full-time member of the Frag Doll team. 


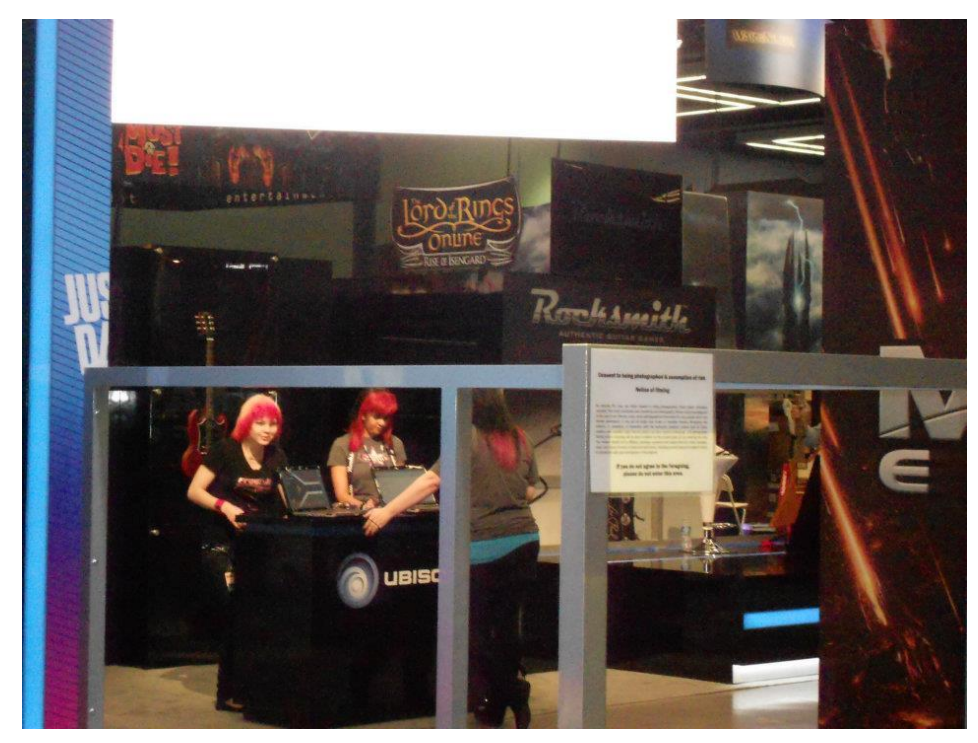

Figure 39: The researcher (center) working the Rocksmith booth at PAX West 2011. The booth consisted of approximately six to eight stations where an attendee and a member of the Rocksmith development team or Frag Doll would guide them through a playthrough of one song or a mini-game from Rocksmith's “Guitarcade.” The marketing efforts emphasized the game's commitment to authenticity. Giveaways at the 2011 booth included a branded guitar strap for people who preordered the game (which I wore as suspenders as pictured above), as well as a guitar that was raffled off at the end of the show. Outside the booth was a small riser that served as a stage. During scheduled times, a member of the Rocksmith development team would show off the game on an overhead screen while talking to passersby on a headset microphone. Every once in a while, the developer would use a virtuosic guitar solo to attract people to the booth.

When the developer wasn't displaying his prowess, a host would be up on the stage talking to attendees about the game over a loudspeaker and encouraging them to come to the booth for a playthrough and a preorder. He was not a Ubisoft employee but had been hired from an outside agency to promote the game on stage. The lines he used 
to attract people to the booth were generally innocuous, informing people about pre-order incentives as well at the raffle going on that weekend. One refrain, however, stood out, in which he repeatedly called Rocksmith, “...the game [popular video game news site] IGN says is guaranteed to get you laid!" While I couldn't find a quote or the source of this particular claim (that news site IGN considered the game an aphrodisiac), I did find the claim familiar as a musician who's often heard that the reason (typically) men take up the guitar or join a band is to woo women. At the end of the event, a Gibson guitar, previously behind glass, was raffled off to one person who became eligible through a preorder incentive, but not before I was called on stage to sing Bon Jovi's “Livin' on a Prayer" in order to attract and entertain the crowd waiting for the raffle result.

Rocking the block: Rocksmith at the Capitol Hill Block Party

In July of 2012, Ubisoft requested my assistance as a Frag Doll Cadette to show Rocksmith off at the Capitol Hill Block Party (CHBP), a multi-day music festival in Seattle, WA. The festival took place across approximately six blocks of the Capitol Hill neighborhood. Ubisoft had a designated tent in the middle of a blocked-off street (shown in Figure 40) which was a straight shot to the main stage where artists such as Diplo, Allen Stone, and Fitz \& the Tantrums performed. In the tent were four game stations with consoles and monitors as well as an Epiphone guitar (Gibson's lower end model) for each station. 


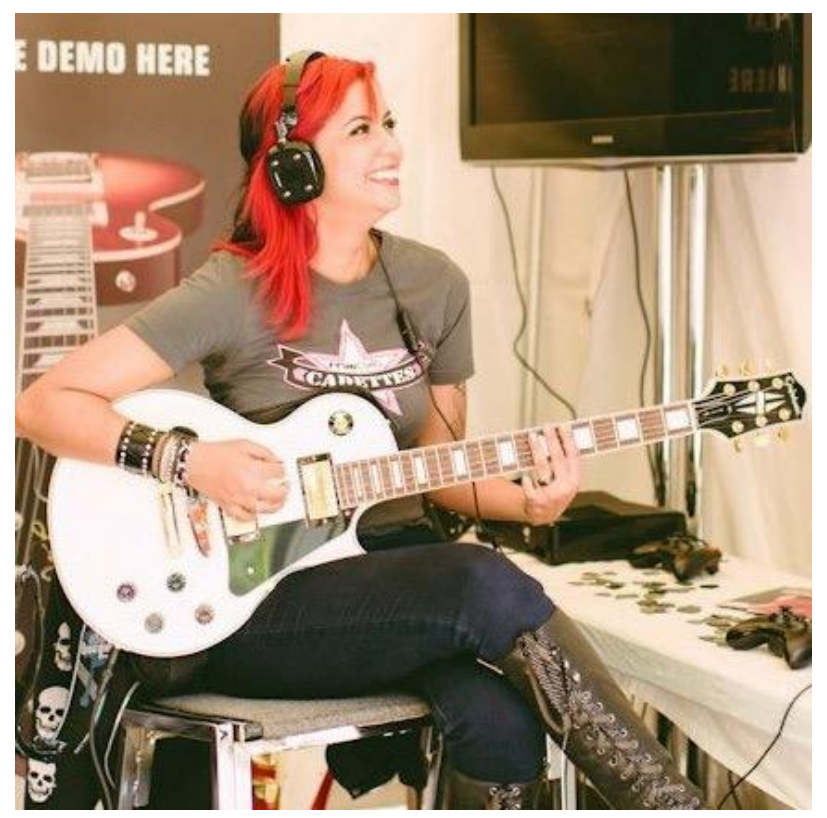

Figure 40: The researcher demonstrating Rocksmith in Capitol Hill (Seattle, WA). Note the branded guitar picks used for swag on the table to the right.

With the booth positioned at a music festival rather than a video game convention, the audiences were markedly different. The PAX booth was positioned before the game's release and encouraged pre-orders through the raffle of an expensive item. The CHBP experience came almost a year after the game's release, with the goal being awareness outside the usual video game circles. It was the difference between "play this video game that teaches you a real skill," and "here's a unique way to learn how to play guitar." One could see how having the game at a music festival makes the experience and goal of the game more tangible---one day, if you play the game enough, you too could be on a stage similar to this one just a few hundred yards away.

It may have been the music-heavy environment that contributed to a difference in the playerbase. I found that, throughout the course of the weekend, I had a more even mix of musicians and non-musicians than I would have seen at a PAX, although it was a relatively lean activity-I personally assisted no more than about 30 people each day of 
the show. I also noticed that musicians experienced the game differently than nonmusicians, having difficulty with the interface and reading the notes as they came down the noteway. While the interface with the scrolling notes is a callback to games such as Guitar Hero and Rock Band, it is possible that a) musicians aren't as familiar with playing those games, or b) there is a fundamental disconnect between their training (whether it consists of sight-reading or tablature) and the way notes appear in the game.

One important observation I made throughout the weekend was that, in general, men were more likely to pick up the guitar and play the game than women were. When I encountered mixed gender pairs, I saw that women were more likely to stand back and watch as men played. Even as I made an effort to oversample the women and actively encourage them to play, especially once a companion had a turn, they often acted with trepidation ("No, that's cool, I'm fine just watching"). Some thought that you needed preexisting guitar knowledge in order to play the game (“Oh, no, he's way better than I am")- despite the point of the game being to teach people how to play guitar from scratch. It felt like the meeting in the middle of the Venn diagram of gender in music and video games illuminated at this very point, with women being reluctant to play both a video game and a guitar, simultaneously, instead opting for the sidelines. It's no wonder. On the first day, a young man approached me about the game, wanting to know more: Attendee: "So, who made this game?"

Me: "Ubisoft! They're also the makers of the Assassin's Creed games, Ghost Recon: Future Soldier, Rainbow Si-“ 
Attendee: "-Not that you play any of those."

\section{Findings}

Right at the outset, my investigation of Rocksmith brought about answers to my research questions - and a rethinking of them - that I did not expect. While I came in with the same assumptions as my Rock Band research (little set list representation, potentially gendered hard-coding in the text, etc.), I found that the nature of the game, with its lack of avatar customization and player character in general, made me seek the answers to my research questions in different ways.

I did not have to, for example, come up with examples of hard-coded gender for Rocksmith - they were delivered to me personally by the developers, who, as soon as they learned my subject of study, tipped their hand as to how the environment and user interface of the game contributed to a heavily-skewed male playerbase. Rocksmith and Rocksmith 2014 are gendered, in different ways: one with a user interface that plays into smoky masculine stereotypes, and one that is less reliant on such tropes in order to be more inviting. One could say the switch in 2014 incorporated some of the "games for girls" checklist of features that developer Brenda Laurel (2008) once proposed:

exploration rather than a fixed path, an end to hierarchical scoring, and everyday settings rooted in reality $(2008: 12)$.

Unfortunately, the analysis is not as glowing when it comes to the set list. The question of hard-coding gender is answered with a set list of musicians that skew even more heavily towards men than Rock Band did (10\% women vs. 16\%). In taking its cues from the music industry, Rocksmith also replicated the same reliance of painting women 
as vocalists, which is damning for a game that aims for the player to become a musician in their own right — but does not teach vocals. To wit, it is a faithful replication of how far women had to stay away from each other in an industry that refused to play women back to back on rock radio, or once told singer-songwriter Sarah McLachlan that "no one...would pay to see two women on the same bill (Garr 1992:412).” McLachlan went on to found Lilith Fair, one of the highest-grossing festivals of all time (which happened to feature all women), but Rocksmith players do not yet have too much of a chance to see much variety when they try to construct their own version in-game.

In the absence of avatar customization or pages and pages of loading screen text to work with, I sought other ways of answering the questions of the gendering of Rocksmith and opportunities for subversion, and I found them-in marketing. This made me rethink the notion of "hard-coding," as it was born out of an idea that the game itself - the digital code, art, and sound design that comprise it, were the only places where I could analyze it. However, I was missing a key feature — marketing — which constructs the game's connotations and expectations before it is even purchased, much less played.

Their use of the Frag Doll crew to sell the original was limited-while women were at the booth and served to attract customers, there were few to actually show off the game itself. That, coupled with the repeated refrain that the game would get you laid, did not lend towards the most gender-inclusive advertising for the game. While the use of women to promote the game and actually play the guitar while doing it increased after launch, it's possible that the effect was not felt until the announcement of the revamped 
2014 version, complete with new aesthetic. In this way, marketing can play a role in hard-coding gender into a game without actually touching code itself.

I hope that these findings, particularly the methodology, reaches other games researchers and entices them to look beyond the game-as-text and seek developer interviews as well as a more holistic look at marketing practices to produce a thicker description of what a game represents. Whether it's reifying or subverting expectations about authorial intent, there is a lot to be said about how teams of developers from multiple disciplines work together to produce a product. There is also a lesson to be learned in treating games as static entities. Now, more than ever, games are frequently works in progress, and it is increasingly necessary to be conscious of how a game can change through downloadable content and updates through game patches - in the case of Rocksmith, more inclusive hiring after the game's release has led to sourcing more women musicians for the set list.

Unfortunately, there is potentially only one chance to make a first impression, and developers, too, must be conscious of how marketing aids in setting expectations and how the game's content at launch meets them-more inclusive marketing and hiring earlier in the process may have led to a considerably more diverse and numerous playerbase the first time around, rather than feeling the need to win more players through downloadable content long after the fact. 


\section{THE PLAYERS: BUTTONS, STRINGS, AND A FLIP OF THE HAIR}

The third point of the creator-game-player framework I wanted to explore in this dissertation was the role of the player. The creators borrow from their own experiences as well as their own research and education to collaborate with a team to produce a game, a text that can be read and analyzed. However, a game cam become something else entirely in the players' hands, where it is their role as audience and participant to craft a unique experience through their play sessions and potentially create opportunities for subversion.

In the case of music games, I aimed to look at the players exclusively for the answer to my third research question, which addresses opportunities for subversion, wherewithal of subversion of gender norms, and whether these can be considered effective resistances. I imagined going into the project that gaming code was more fixed, printed on a disc or delivered via a code, while players and their individual experiences would be the source of subversion and change. In order to tease this out, I embarked on informal interviews, a player survey, participant observation of public play sessions, and an interview with a professional music game competitor to seek out the answers.

\section{Our Avatars, Ourselves}

After my Xbox Live gamer spotlight experience, I was inspired to conduct informal interviews with 8 self-identified gamers to see whether their Xbox Live avatars cemented this link, both in gender and overall appearance. Most of my informants $(n=$ 5), especially the women, stated that they tried to make their avatars' facial features, skin color, body type, and hair color match them as much as possible. They may have game- 
specific costumes (such as Catwoman, or a harlequin character available from the Assassin's Creed franchise), but otherwise tend to match their owners in appearance:

- It looks like me! Or at least as close as it can... (Respondent 1)

- My [Xbox] avatar looks as much like me as I can get it: blonde hair, blue eyes, similar hairstyle, etc. I just wish I could make my avatar's boobs bigger lol! (Respondent 3)

- ...Oh, the usual...I dress her as me to show everyone that you can be you anywhere ya go! (Respondent 4)

What happens when this relationship is fraudulent, that is, if the avatar and reallife genders do not match up? Because my informants overwhelmingly cemented the link in their Xbox Live avatars, I expanded the question to include situations where they engaged in cross-gender play. When given the choice, the women tended to link genders with their avatars with few exceptions: One informant told me that the man she was playing with, upon the reveal, had expressed that he had "won the jackpot," finding a girl who also played video games. Another changed genders because they feared comments otherwise: "I always used dude avatars until I...found my girl self in gaming. You don't have to deal with idiots as often when your character looks like a dude.” The genderswitch is justified, as evidenced by a comment received in response to one woman's gender-matched and even race-matched avatars: "I once received a message from a guy who said my avatar looked hot and he bet I was too lol."

These responses are often the very reason men choose to cross genders in games such as the massively multiplayer online role-playing game World of Warcraft. A couple 
of the men in the interviews expressed that, in these games, female avatars are given preferential treatment, including powerful in-game items, in-game gold, and aid during quests. Others used cross-gender play as a means to 'troll' others (online mischief). A rare answer was a self-identified straight man who used cross-gender play as a means to show his support for female characters, which are less numerous. Regardless of the reasoning behind cross-gender play for these men, the results upon discovery that female avatars are being controlled my men are laced with homophobia and transphobia:

- Some dude sent me a rose in my mailbox. When I [private messaged] him telling him I was a guy, he went ballistic. Telling me I was some sort of transvestite homosexual and such, in obviously less elegant and PC language...calling me a 'confused faggot.' (Respondent 1)

- It was mainly 'You Queer' and 'Fucking Fag' and along the like of that...people overact even back in the day when I would play a female human...I would get mail from dudes expecting me to call them [in real life] ...then when I say I'm a dude...the same thing comes back around. (Respondent 2)

An explanation for the harsh reactions, aside from the aforementioned typical responses to non-normative avatar performances is found in Currah \& Moore's discussion of Erving Goffman's presentation of self: there is a 'promissory character,' a moral character (Goffman in Currah \& Moore 2004) in one's self-presentation. The avatar's programming and presentation as female is a "promissory document... about an 
individual's body... what is in fact social gender is assumed to guarantee a

correspondence between one's present body, its past, and the gender presentation one puts out (119)." It is the natural attitude linking genitals and gender that can be blamed for this trust, which is so strong, that it bleeds into a world where one can choose from an increasingly varied set of gender performances with a click of a mouse.

\section{The Player-Performer: A Survey}

I conducted a player survey in July 2014 where I specifically asked about player's experiences with both Rock Band and Rocksmith, as well as perceptions of gender, race, and sexuality in these game spaces and games in general. The survey was deployed via survey site SurveyMonkey, and participants were found via convenience sampling: sharing on social media as well as forums I frequented. After approximately two weeks of gathering respondents, there were a total of 163 participants. Their race/ethnicity breakdown (see Figure 41) is as follows:

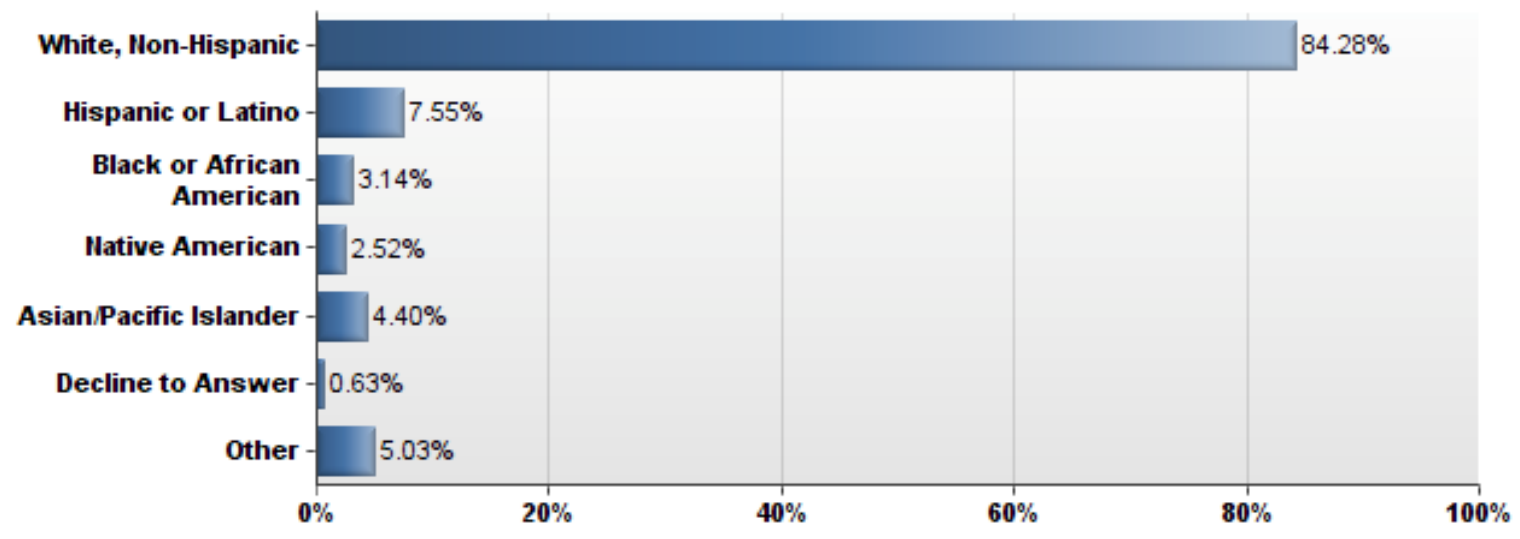

Figure 41: Racial/ethnic breakdown of respondents. Image rendered by SurveyMonkey. 
The majority of respondents $(84.28 \%, \mathrm{n}=137)$ identified as White, Non-Hispanic. Of the respondents that answered "Other $(5.05 \%, \mathrm{n}=8)$ " the write-in field yielded the following:

- The Whitest of the White Girls (Respondent 11)

- Middle eastern (Respondent 37)

- Australian (Respondent 46)

- Mixed White and Hispanic (Respondent 67)

- Do we really need to make this a 'race' thing? (Respondent 70)

- Human (Respondent 79)

- White British (Respondent 83)

When asked about their gender identity (Figure 42), the results were as follows:

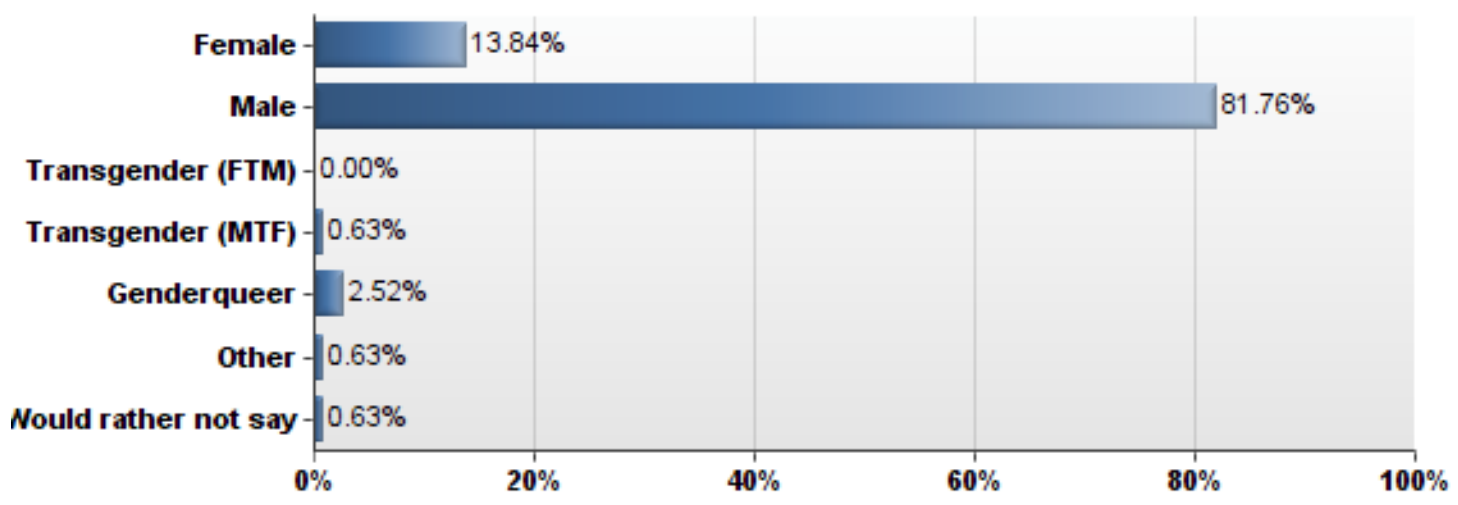

Figure 42: Gender breakdown of respondents, with categories already problematically out of date. Image rendered in SurveyMonkey.

The one person who selected "Other" wrote in their gender identity as "agender." Despite the distribution of the survey in an all-woman gaming group and shared by a few prominent women games journalists, the respondents were overwhelmingly (82\%, $\mathrm{n}=133)$ male. 
Perceived target demographics

Respondents were asked to write who they thought was the target audience for both Rock Band and Rocksmith. For Rocksmith, 12\% of respondents thought the game specifically targeted men:

- Age 15-50. Male. Musically enclined [sic] gamer (Respondent 150)

- Honestly, it comes off more like it's marketed to older men. What I've seen and heard of it on YouTube, a lot of the content feels like it would resonate more with that demographic (Respondent 21)

- $13-55$ probably mostly male but maybe a $80 / 20$ split of male to female (Respondent 147).

Rock Band perceptions were more likely to deliver a response of "all genders" in response to the demographic ( $31 \%$ of gendered responses vs $10 \%$ with Rocksmith), but a majority of responses that mention gender specifically as part of the demographic states that the game caters to male players:

- All ages and genders. Song choices seem to be a bit more in favor of male tastes (Respondent 9).

- Male $18-24 \ldots$ is what I assume, as everything is apparently targeted to that group (Respondent 22).

- Stereotypical gamers: SWM [Straight White Men] (Respondent 156) 
More often than not, respondents found that these games were catered to men just by nature of being a video game, although Rocksmith may be more likely to be listed as a male-dominated game due to song choice, the emphasis on authentic guitar experiences, or marketing materials. Most of the players had some experience with an instrument (88\%), with most concentrating on instruments typically found represented in Rock Band and Rocksmith (guitar, bass, drums, vocals).

\section{Harassment in video game spaces}

$17 \%(\mathrm{n}=26)$ of players said they have had negative experiences with video games due to their race, gender, or sexuality. When asked to describe their negative experiences, most were gender-based ( $61 \%$ of harassment suffer respondents, $n=16)$, although some expressed negative reactions due to race and sexuality (26\% and $11 \%$ of harassment sufferers, respectively). Online play for women often draws reactions which reference gendered tropes of housework and domesticity:

- Guys used to ask me to make them a sandwich. Or ask how I could play Xbox from the kitchen, or tell me to talk to them when my "balls dropped" cause in disbelief that was a girl they would think I was a 10 year old boy (Respondent 26).

- Because I am female I constantly get the "go back to the kitchen and make me a sammich" treatment (Respondent 33).

- Being a female, male gamers often like to tell me that they will rape me or something because they got beat. Other times I am told to go back to the kitchen or something (Respondent 29). 
The result of these negative experiences is to hide who they are or "brush it off." Negative online behavior has been normalized to the point of being an almost accepted evil: "Online gaming is an absolute cesspool that I recommend no one participate in with a headset."

In contrast, only seven respondents noted that they had had negative experiences as a result of the games themselves or the community:

- In a similar way that I experienced it with online games, the assumption is that as a female player I wouldn't be as good or as interested in competition as male players (Respondent 22).

- I kept getting challenged by a random, cause I was singing better than him on the vocals, he said it wasn't fair cause I'm a girl and could hit the higher notes (Respondent 26).

- I have had people say online that playing Rock Band at a tournament level does not qualify me as a gamer, just like girls who brag about 3-starring all the levels in Angry Birds. I posted some gaming videos on YouTube and sometimes people would comment on my appearance rather than my performance in the game (Respondent 48).

One respondent asserted that it's possible that music games do not suffer the same kind of harassment problems as other game genres because of a lack of support for online communication. These games tend to not support synchronous online multiplayer due to 
issues with latency, which in turn doesn't encourage online communication even with Xbox, PlayStation, or PC platform-level methods. Instead, Rock Band and Rocksmith encourage what is known as couch co-op, where you play cooperatively with people in the same room.

\section{Meta-conversations on the feminist study of games}

In an interesting turn, two respondents expressed negative experiences as a result of the increased examination of video games from a feminist lens:

- Anita Sarkesian (spelling) (sic) I'm a white male and that makes me terrible because games are targeted (sic) toward my demographic (Respondent 110).

- Not because of the games in themselves, but because of the community. People often accuse me of being sexist without me actually doing anything, which is why I often hide my gender and ethnicity if possible. I know that there are bad white men out there that are both racist and sexist, but I'm not one of them, so I don't think it's fair to judge me in the same light (Respondent 80).

Additionally, some of the most compelling responses did not address the games, so much as the study itself. One respondent expressed that his status as the hegemonic gaming demographic may not have made his as well-suited to provide good responses to the survey: "I have a feeling my answers were not very helpful, since gender did not even occur to me when playing these games (and from what I've seen of Rocksmith). Not surprising for a cis gendered white male, though, 
right?" His thoughts are reflective of an increased sensitivity to conversations on diversity and inclusion in video game spaces.

In addition to (and in response to) this increased sensitivity, there was one respondent who asked me to "Please [do] not sink to the level of Anita Sarkeesian (Respondent 86)." Anita Sarkeesian is a feminist media critic who came under fire for her Kickstarter-funded video series Tropes vs. Women in Video Games. The video series focuses on the numerous (potentially problematic) ways women are portrayed in video games. The series was derided by a subset of gamers as being part of a call to censor, or an effort to make games more politically correct and sanitized of all sexual content. Some accused her and women like her (also known derisively as "Social Justice Warriors") of trying to sink the video game industry altogether. By virtue of being a feminist researcher, I am instantly considered a threat to the male-dominated space of video games with an aim to destroy them or sanitize them as opposed to encouraging more inclusive design.

\section{"Rocking" Out in Public}

As part of my qualitative analysis, I chose to observe players at different sites of public performance, to see the ways in which players tend to emulate rock musicians' movements when playing Rock Band or Rocksmith. I chose two kinds of sites: Rock Band nights at a club that hosts live music regularly, and video game conventions (which also host live music, but that is not their primary focus). I attended one Rock Band night at a club once known as Transit Lounge in the downtown Miami area. I also observed people using designated public play areas at two different video game consumer and fan 
conventions, the Penny Arcade Expo (PAX) in Seattle, and the Music and Gaming Festival (MAGFest) in Maryland.

This look at public performance is an extension of the views of scholars Kiri Miller (2012) who confirms that playing these games are more than just pressing buttons, but are collaborative performances in themselves: "each player collaborates with the game designers to turn code into a virtual performance, while remaining aware that millions of other players have engaged in the same endeavor (5)." Karen Collins (2013) extends this view to actual physical performance:

Through promoting performance, these types of games strengthen the role-playing aspects of the game, encouraging a kind of rock performative excess and rewarding the player for these gestures (raising the guitar to enter Star Mode and so on). Shy players may in this way mimic the kinds of performative activities that they witness popular stars enact and though this physical mimicry may be able to live that performance mentally (69).

To wit, I experienced another interpretation of this performative notion in a more private setting. I had my husband assist in capturing game footage to replace footage I had lost during my initial playthrough, in order to grab reliable screenshots for this dissertation. As he played, I noticed his playstyle was different. We have both been musicians for a couple of decades, and his experience is primarily guitar-based. I saw that, as he took on my guitar-playing avatar, he frequently used the whammy bar on the plastic guitar to shift the pitch of the notes he played to bend them in and out of key. He wanted to see if that would in any way affect his score. It didn't, even though he often took the song to a...rather displeasing dissonant place (mostly to get a laugh out of me). Soon after he 
began doing that, he got a loading screen tip encouraging the use of the whammy bar to customize his sound. In addition, some of the songs end with a section allowing you to perform a freestyle solo (see Figure 43), and you can add effects to the sound of your guitar, even if the song itself doesn't use them:

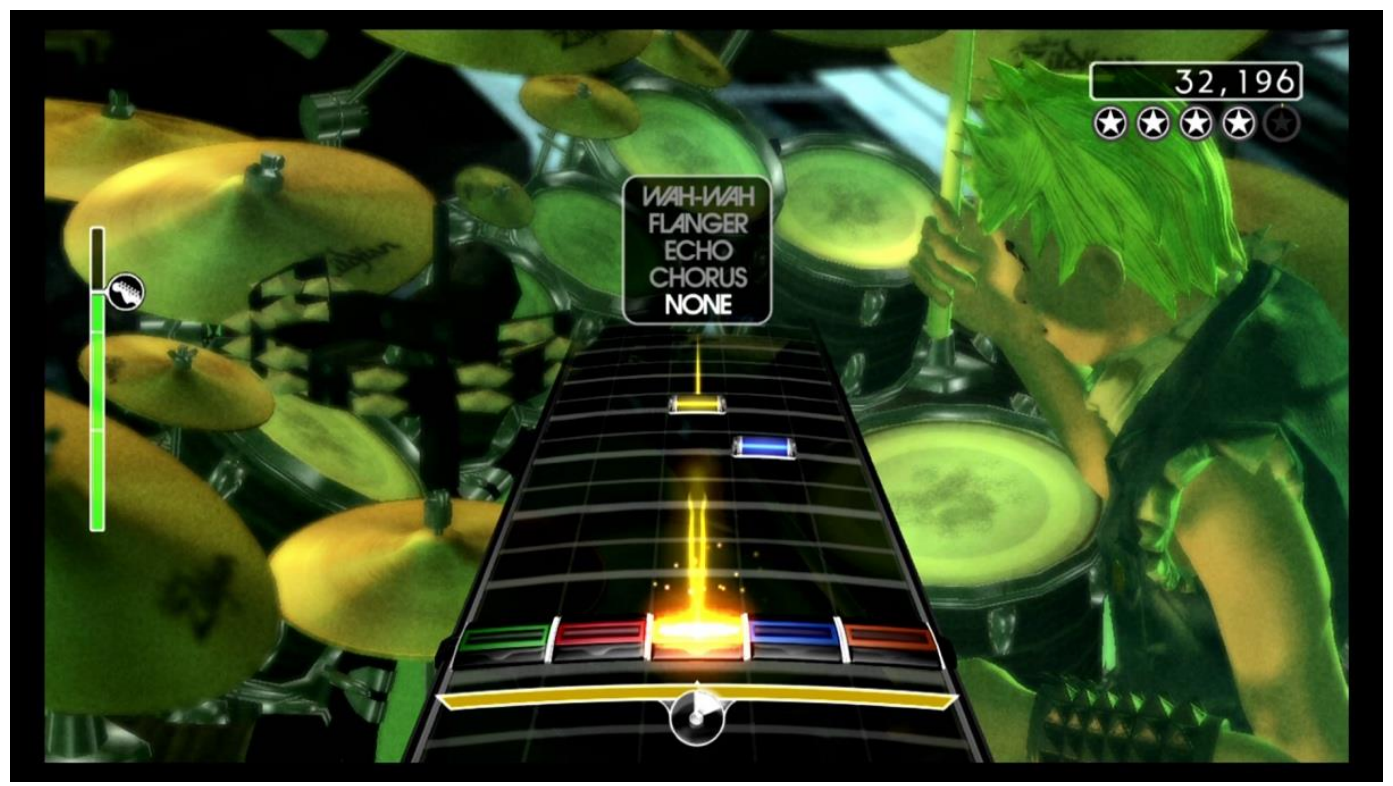

Figure 43: Embellish the sound of the song through whammy bars or effects to make the song your own. Screenshot from Rock Band.

These are not the only ways in which one can customize a song's performance-which is why I turned to public performance in front of crowds. My brief observances of public play as well as an interview with a competitive music game player serve as a way to determine what these movements are, how closely they replicate traditional rock choreography, and in what ways gender is performed through these public play sessions.

The first site was a Rock Band night at downtown Miami’s Transit Lounge (now Blackbird Ordinary) in February 2011. I arrived close to midnight, where I saw the plastic instruments (and one real microphone) set up on the stage. The event was a 
competition in which the best band would win a $\$ 50$ bar tab. "Best" was determined via quantitative methods: a mix of the number of stars each person earned, along with total band stars and total band score. The purely quantitative nature of the scoring system did not, however, detract most people from performing what could be considered canonical rock choreography.

Out of the five bands observed that night, four of them displayed some semblance of "rock performance." The first band, performing Green Day's "Basketcase," displayed interaction between the vocalist and guitar player during the guitar solo, where the vocalist plays a more supportive role and eggs the guitar player on. In addition, the band decided on kicks and sticking their tongues out as signature moves, an aggressive and performatively ugly gesture that belied the punk origins of the band they were covering (Eileraas 1997).

While there were more men than women competing on stage, the instrumentation among the three women observed (versus 14 men) was spread evenly between bass, guitar, and vocals respectively. The last band featured a woman lead singer, choosing "Spiderwebs" by No Doubt after a long deliberation. Along with kicks and stomping her feet, the singer placed a finger to her ear, a move that I recognized as an 'I' $m$ trying to hear myself" move. By blocking the auditory canal, one is able to hear one's voice inside their heads, and it is a move famously employed by female singers (and often lampooned because of its diva implications). The singer, I found out later, was a singer in a local rock band in her own right. In a brief chat after she left the stage, she revealed to me that she had also experienced some of the frustrations regarding being a woman in a rock band, including not being taken seriously as a musician versus her male counterparts. 
At the Penny Arcade Expo East in April 2014, there was a riser set up in the convention center with a Rock Band set-up featuring guitar/bass, drums, keyboards, and multiple microphones. For the most part, it appears that the focus is more score-centric than performance-centric. Players tended to stare at the screen to focus on the gameplay rather than engage in "rocking out"-type behavior. When players do engage in performance behavior, they headbanged, nodded their heads to the beat, kicked, and employed other related motions reminiscent of live music performance (such as making a fist and pumping it to the beat). In terms of gender demographics, more men than women tend to attend these events. However, out of all of the public play spaces observed, more women were seen playing Rock Band than any of the Guitar Hero or Rocksmith stations observed.

\section{When Players Compete: An Interview}

Players in the July 2014 survey were not likely to compete in Rock Band competitions. Competitive gaming, also known as esports, is currently centered around first-person shooters (FPSes), multiplayer online battle arenas (MOBAs), and sports games rather than rhythm games. When music gaming was at its peak, players could compete in national-level Guitar Hero and Rock Band tournaments, as well as recording their attempts at perfect scores and uploading them to YouTube.

I had the opportunity to interview one such competitor, "StarSlay3r," about her experiences as a Guitar Hero and Rock Band competitive gamer. Her story of discovering video games follows a familiar trajectory in which she received a console for Christmas 
and grew up playing. Once she found out that people could play video games professionally and make money doing it, she pursued competitive play.

In order to get a grasp of the competitive scene in Guitar Hero and Rock Band, she would seek out the Scorehero.com forums. Scorehero.com is "where most of the top players would go to discuss guitar hero on a competitive level from strategy to star path charts to tournaments and general advice (StarSlay3r, Personal Correspondence, 2014)." Word of mouth also played a role in entering tournaments, which speaks to a more insular experience where one would have to build networks through competitive play in order to get an invitation to compete.

Practice sessions for StarSlay3r included sometimes putting in 40-60 hours a week and balancing full-time work in order to compete. Her instrument of choice? Guitar. She explains:

Guitar is where it was at for all competitions. It was very rare to find a competition involving the whole band, and even more rare to find a tournament revolving around any other single instrument in the game. Playing the guitar was fun and challenging (StarSlay3r, 2014).

Her suggestion that it was rare to find competitions with other instruments is telling, as it points to continued reification of the guitar's role as signature instrument of the rock genre, even in a video game competition. It seems like a majority of her competitions were using the guitar, and we also score-based. She describes playing rhythm games like Guitar Hero or Rock Band as a different animal than fighting games or FPSes, citing that one mistake at the beginning of a song could be the difference between a win and a loss. Score-based play leans into entelechial motivation as described by Charles Soukup 
(2007), which is based on a desire for "completeness, finishedness, and perfection (166)."

It is this perfection that she describes as being markedly different than other genres: It's not like a fighting game where you can nail a combo breaker and save yourself and turn it around. In Guitar Hero missing 1 note at the beginning of the song usually means you lose that song and there's no coming back unless your opponent drastically messes up somehow (2014).

This type of competition, which was purely score-based, involved very little physical movement on her part: "I couldn't even get used to playing Guitar Hero standing up let alone think about moving without messing up (2014)." This eventually evolved as more competitions arose which also judged entrants on "showmanship." She eventually found that "rocking out," as she describes it, became an integral part of the competition. "Rocking out" is mainly defines as paying attention the audience rather than the screen as well as engaging in physical movements that are familiar as classic rock choreography: ...singing along, headbanging, etc. Basically everything a REAL guitarist does when they're playing at a concert. I remember back when I first started doing showmanship stuff people used to laugh at me for being "too into the game" and trying to "act like it's a real guitar", but later on down the line I began getting hired by companies exactly for that reason! (2014)

Despite her prowess, she found herself subject to a lot of the perils that often befall women in the gaming public eye:

Guys will either totally fall head over heels in love with me for being their "dream girl" who is good at games and decent looking, or they will harass me to no end 
asking me to show my boobs on stream or claim I only got the things I have (sponsorships, etc.) simply because I am a girl or I have not been taken seriously as a player at times as people simply view me as a "cheerleader" at events/tournaments judging me off appearances and not actually watching me play (2014).

In that last exchange, StarSlay3r reveals that she has, in one fell swoop, fallen into all three of the categories Taylor, Jenson, and de Castell (2009) observe that exist for women in esports: "Cheerleaders, booth babes, and Halo [or Guitar Hero in this case] hoes (2009:239)." The cheerleader's role is to hold an exclusively supportive role, the booth babe serves as sexualized marketing prop, and the Halo hoe is the groupie whose only purpose is to pick up the stars. In all three of these cases, they are placed in direct heteronormative opposition to the male professional gamer. In none of these cases is there room for a woman to be there just to play---another stark similarity to rock music, in which women serve to be fans, sell guitar amps, or be sexual muses, but not to actually create music.

What is remarkable, however, is that she did not experience the same sort of harassment or skepticism within the rhythm game community, stating that there were enough women involved that the novelty (and some of the scrutiny that comes with it) was not as persistent in this particular space. StarSlay3r specifically cites representation within the community as a source of a more inclusive. While this provides a potential solution and possible example for video game genres and playerbases as a whole, it may not extend to competitive scenes in other genres as long as esports maintains an attitude that aims to emulate mainstream sports discourses that "[base] continued male 
domination (and privilege) on an alleged physiological superiority of men over women (2009:249).”

\section{Findings}

I sought out to answer the question of opportunities for subversion by examining music video game players. The surveys, interviews, and observation of public play provided an answer to the third question, but it also provided support for my other research questions and led to a re-examination of some of my preconceived notions coming into this project.

The first point of support came in the survey looking at how players identify with their avatars. My findings from informal interviews supported existing literature on avatar identification (Banakou 2010; Sioni et. al. 2017; Yee 2006). Making avatars that looked like themselves — or, more importantly, amplified versions of themselvessupports literature that links having these avatars to greater senses of self-efficacy. This echoes a lot of what was found in my look at Rock Band avatars: clothing and makeup options than can allow you to become this stylized form of self (albeit, with some limitations such as shade range and still holding onto a gender binary).

When it comes to actual subversion, I found that players provided their own by virtue of showing up to play, with StarSlay3r as a prime example. Despite discussions of women in the gaming industry (as developers, characters, and players) becoming more prevalent over the last decade, attitudes still persist regarding women's inclination (or lack thereof) to play and create games. To this end, it is still somewhat revolutionary for 
women like StarSlay3r to not only play games, but to play them professionally at a high level of skill.

For all of its support, however, this portion of the study made me reexamine both my questions and methodology. While the idea of "just existing publicly" can provide a form of subversion, it was one of the only forms of subversion I found. In fact, I saw that, in the process of answering the third research question on subversion, I ended up seeing just how effective Rock Band emulates and facilitates an "authentic" rock public performance (thus contributing an answer to my first research question). When playing Rock Band, you can move the plastic instruments in such a way as to perform double duty: the movements emulate rock choreography, thus adding to the immersion into the rock fantasy, but performing them successfully earns you more points. By performing these movements accurately in a public play setting (especially at a video game convention), players perform rock authenticity as well as "gamer" authenticity in one flip of a plastic guitar neck. Ultimately, then, I answered the question of how Rock Band emulates existing rock and gaming discourses in the process of trying to find gendersubversive spaces.

By the time I sought out these public performance spaces, they had significantly dwindled to free play spaces at conventions and a lucky local find of a Rock Band night. Gone were the days of major competitions for cash prizes with authentic developer and instrument sponsors, replaced with a party game that fills public space at a convention for casual social play, bringing the living room to a bigger audience. In this case, it may have behooved me to seek out more in-depth experiences, including more in-depth interviews 
with professional players, rather than to cover a wide swath of similar public play spaces as different conventions over the last few years.

Methodologically, this is a cautionary tale for similarly-minded academics who chase less mainstream genres of games. The music genre is an example of a genre that reached wide mainstream appeal followed by a decline that was just as swift. As such, it may be more difficult to conduct research when a genre is on a downswing in popularity (unless the downswing itself is the subject of study).

It is also a cautionary tale for those that aim to conduct research on games from a feminist lens. While I attempted to maintain as much of a critical distance as possible, I still found myself in the shadow of Gamergate, with my research and aspects of my personal life put greatly at risk for the audacity to speak up and conduct this research openly. While the Gamergate movement is not at its 2014 fever pitch, the effects are still felt, so taking measures to protect oneself is still as necessary now as it was when I first began this study in 2009 . 


\section{DISCUSSION, LIMITATIONS, AND CONTRIBUTIONS}

This research took place over more than a decade, between previous forays into feminist musicology and this current research project, in addition to over two decades as both a musician and video game enthusiast which informed the entire project. Through the exploration of the games, the creators, the players, and myself as researcher/musician/gamer, I aimed to shine a light on a relatively underrepresented genre of video game while applying a well-trodden feminist lens in a more thorough way. I also sought to answer the following questions:

1. How are Rock Band and Rocksmith gendered? How do they emulate or subvert gendered music or gaming discourses?

2. How are gender norms hardwired into the game itself?

3. What possibilities exist for the subversion of gender norms? Where do these resistances occur (solo performance, small groups, public play/competitions)? Are they effective resistances? Why/why not?

\section{Rock Band and Rocksmith: Gendered Cover Versions}

Rock Band and Rocksmith are, in general, gendered productions. I would argue that it is difficult, if not impossible, to produce a cultural work absent of the political, much less an interactive product, and even less an interactive product experienced in first-person or via an avatar of your own design. Regardless of authorial intent, a work of entertainment is not absent of politics, much less identity politics - particularly a game that makes you choose a gender for your avatar as part of the game, or one that specifically releases a "Women Who Rock" song pack. 
Rock Band provided the easiest answers to the questions of the nature of the gendered game, as you are asked to choose between a gender binary for your rocker avatar, and then dress them up in mostly normative clothing. Both games share a perfect replication of a mainstream American rock canon that is somewhat devoid of women, much less musicians. Rocksmith presented a unique opportunity for a direct comparison between a more masculine-coded design (the dark studio, the leather-clad instructor) and an attempt at a more gender-inclusive design. While I do not have numbers to show whether this was effective, this researcher sees it as a much-needed and welcome change.

\section{Gender as Hard-Coded}

Gender norms are hardwired into the games themselves via their design and gameplay elements. In fact, my interviews with developers from Harmonix and Ubisoft point to ways in which developers actively try and make games more inclusive, whether that is through narrative or user interface design. Aside from these more deliberate versions of gender hard-coding are ones that are borne from attempts at replications of music culture as well as game design conventions and mainstream gender norms. In particular, women's subservience and supportive role to male heroes as well as a dampened desire to take part in technology (Whether that is a controller or a musical instrument). The makeup of setlists, the inclusion of a "sound guy" as part of your personnel, the clothes you wear-all create a discourse of what it means to be in a rock band, and who is welcome to join. While both these games exhibited intentional design with an eye towards inclusivity, it is necessarily to have more diverse points of view in 
all aspects of game design in order to catch unintentional or well-meaning but poorly executed attempts.

Because Rocksmith did not offer customizable avatars to perform a comparative analysis, my unique proximity to the development of the game led me to lend a closer eye to video game marketing. One of my biggest surprises was in seeing just how much or a role marketing can play in the service of hard-coding gender into a game, instead of looking at the game itself.

If the game is the lead singer, the marketing is an accompanist, supporting and enhancing aspects of the performance while silencing others. When a game is marketed as real, or as a pedagogical tool, or as being guaranteed to get you laid, it is catering to specific audiences and outlines who this game is for. From experience, I know that marketing can often times be at odds with game design intent — and what is a gamechanging feature to the person that made it is not necessarily the thing that will be attractive to the widest audience. My recommendation is for diverse perspectives on both the design and marketing side in order to avoid alienation of potential markets. This is not merely a play at getting companies to embrace diversity in order to fill their coffers-it is a necessary part of creating more responsible content, because representation matters. It may not matter how many skin tones you can choose for your avatar, or how much of a gender-balanced setlist you have in your game, if your marketing ends up turning people off from experiencing the game in the first place. 


\section{Subversion in Song?}

While I expected much of the resistances to gendered rock and video game discourses to come from public play spaces, a bulk of them actually came from representation in development spaces (in the case of Harmonix's gender-conscious writer) and the use of user research to influence design (in the case of Ubisoft San Francisco). In general, subverting gender norms through avatar selection and customization is fraught, as seen through player surveys, as those opportunities can be met with potential harassment. For music games, however, this becomes less likely and public performance can be the source of subversion as many people regardless of gender identity can either emulate rock movement or stay still and focus on achieving a high score. Public play, especially expert public play, can become a source of resistance, as seen in Starslay3r's example. Her experience as a visible member of the esports community provided much-needed representation in a world that still struggles with the roles women play. On the other hand, how effective can this resistance be when it's as part of an already inclusive (according to her) subgenre of games, and if it takes expert-

level play to be taken seriously as a woman player-in fact, it is merely a reflection of the state of affairs in both rock music and video games.

There is, however, a flip side to this. With Clawson (1999) arguing that age of first access to music production may be as much of a predictor of future performance as representation, it is entirely possible that a more inclusive rhythm game community could be the basis for more women gaining access to music performance (albeit simulated) earlier in their lives and provide inspiration for them to create their own music — and perhaps even their own video games. 
Verse, Chorus, Solo, Double Chorus, Fade Out: Subverting Expected Formulas

Upon entering this study, I expected for these three questions to be summed up in their entirety by the methods set out. Of course, as I imagine with all research projects of this length and magnitude, it ended up being far more nuanced and gray than I initially thought. The conversations with developers proved to be the most eye-opening portion of the study. When I read previous academic forays into gender and games that treated games as texts devoid of their creator and player contexts, I expected to encounter developers that would tilt their heads at any mention of the word "gender," or outright defensiveness and denial that their products could be even mentioned within this conversation. What I encountered were writers and designers for both Harmonix and Ubisoft that made conscious efforts to make their games as inclusive as possible. My study shows how successful those attempts at subversion are-some attempts more than others.

Another point of insight that made me rethink my questions was in the treatment of games as somehow being crystallized in amber while taking on the theoretical framework that gender is in constant state of negotiation between performer and audience, and that gender is not premade or fixed, but in a constant state of being written and rewritten. So, too, are musical performances, and so too are games. When looking at the creator-game-player dynamic, I predicted this would be true-each player experience is different, and games often provide multiple game experiences (solo vs. multiplayer, a narrative playthrough vs. just jumping into one song, customizing an avatar vs. choosing a preset) that increase the degree to which my experience of a game can be different than 
yours exponentially. It was here that I expected the most fluidity in terms of possibility of subversion.

However, I learned that the creators and the games themselves have just as much malleability. My experience in the game industry over the last few years contributed to the knowledge that games are often reshaped through subsequent patches after their release. When a game hits store shelves, it is no longer an accepted end to their story. Parts of the game that were previously broken or "bugged" get fixed. When it comes to music games, support from the developers in the form of releasing new music to play can continue years after the game's initial release. As games change, so do the developers, which often stay for a while before moving onto new projects and hiring in a constant state of flux. With those new hires can come new changes to games that were previously unexplored.

At the time of this writing, I learned of a recent hire to Ubisoft who took the same look at the first 1,000 Rocksmith songs and found them lacking in representation. She has been an active champion in trying to source more women artists and artists of colorspecifically guitarist and bassists. Three years after the release of Rocksmith 2014, strides are still being made to make the game more inclusive. However, this is coming three years after the game's release. It may be too little, too late unless another entry to the franchise is planned (with subsequent marketing spend behind it) to introduce a new audience to a more inclusive set list from the get-go.

I still think there is value in analyzing a game in itself. As shown in this study and countless others before mine, games by themselves are not free of gender politics, regardless of authorial intent. However, games and the people who make them can 
change in response to feedback, and the hiring of more people with a mind towards inclusive design is a step towards creating Fullerton et. al.'s "virtuous cycle" that can make better games, regardless of genre (2002:175).

\section{Limitations}

Access to the creators was made easier by establishing connections over years of working and attending industry events. However, there were some obstacles which presented themselves at the point of office visits. Both the Harmonix studio in Cambridge and Ubisoft Studio SF are currently producing games, including unannounced titles and content. Because of this, office tours and access to developers were limited by scheduling and unavailability of locations. As the employees are held to non-disclosure agreements, the interview protocol question of "What does a typical work day look like?" could not be answered in the present tense and had to be amended to some variation of "What would have a typical day looked like during the development of [released title]?" Tours were either carefully structured or cut short as to avoid my seeing content in progress. Even long after the game's release, obtaining access to salient documents such as the Rocksmith player survey was not possible due to it being information that should not be disclosed, lest competitors receive access to it. I am grateful for the access that I was granted in light of the circumstances, however.

On the player side, the participant observation at conventions was not as strong as originally intended due to a wane in competitive Rock Band play. Free play (rather than competitive play) did result in some salient observations, but there could admittedly be a shift in performance style due to the particular focus of a competition. 
I am conscious of the limited role race and class factor into the research. The history of American popular music and specifically rock n' roll is the story of black music being co-opted and sanitized for white audiences (where mainstream success is found). While they originated the music, people of color are not represented in mainstream rock music as much as their white counterparts are. Race also factors into the video game player base as well as development teams: people of color are represented in limited and often stereotypical forms, and games are often marketed (like a significant amount of popular media) to a young, white male demographic. Barriers to entry into the industry for women in general are many and varied. According to Fullerton et. al. (2008), industry surveys list the following factors behind this disparity in the participation of women:

These include extreme working conditions and poor quality of life, a misconception that girls don't play games, the industry practice of making games that makers (most of whom are men) like to play, an unfriendly work environment and "garage hacker" culture, and alienating business practices such as "booth babes" at trade shows (164).

These conditions are likely to hit women of color just as hard if not harder, as a lack of role models in both development and video game representation break the virtuous cycle that Fullerton et. al. strive for. For every Lara Croft you can play and every Brenda Romero you can look up to, there are countless sex workers and criminals in Grand Theft Auto and nameless brown terrorist masses to fight against—-that is, if people of color appear at all. According to Dahya et. al. (2017), this discrepancy can be mediated via early intervention in computer game development education that is "hands-on, student- 
driven, creative, feminist, racially inclusive, and development-focused...modeling expertise by female, nongender conforming, and racially diverse mentors (384-385)."

Until this more feminist approach to game mentorship and early intervention becomes widespread, lack of representation between rock music and mainstream video games causes a double-bind in which the disembodied hands of Rocksmith tutors are white hands playing mostly white songs, the majority of which take inspiration (whether acknowledged or not) from black artists.

Another limitation exists in the form of mere time. In the almost nine years it took to complete this research and write-up, the music game genre has ebbed and flowed, often calling into question the relevancy of this project. The music game genre currently sees life with a yearly release schedule of dance games (Dance Central and Just Dance, also from Harmonix and Ubisoft, respectively), but music simulation games have declined since Rock Band's peak in the early 2000s. While the hope is that this study can inform even more mindful creation of music simulation games, it is not known how many more music simulations there will be to inform.

\section{Contributions and Final Thoughts}

I am heartened, however, by the potential contributions to video game scholarship, gender scholarship, and the video game industry as a whole. In reviewing the literature on both video games and feminist musicology, I found that there is little scholarship on either topic surrounding their intersection, the rock music video game. Feminist musicology has done well to explore gender concerns in both classical and popular music, including artists' inclusion in the canon (or reasons for a lack thereof), 
how they are treated in the press, and performances on stage and screen in the form of music video. I argue that this study of music games presents another site of discovery, where one can analyze virtual bands, their movements, their treatment in text, and their set lists in the same way as you would corporeal bands.

This study also adds another dimension to video game literature, specifically gender discourse analysis of video games. These games are available to younger audiences and don't feature more traditionally explored female characters. As recently at this year, studies by prominent scholars such as Nick Yee (2017) have left out rock music games in breakdowns of player demographics and interests by genre. Even in the subgenre of rock music video game literature, this study may be one of the only ones that place gender at the center of analysis as opposed to educational impact, game design, or pure musicological analysis.

I also believe I have made a contribution through the methods employed in the study, including using activist work in the video game industry as a way of translating these sometimes ivory tower-level concepts of gender performance and sociological impacts of representation to the wider audience, as well as conducting more of an autoethnological look at how I am situated within both rock music and video game industry contexts, and how that impacts the research. I hope that my search within as well as my look towards intentional design, its failures, and player relationships with games can be used not just by academics but also game designers in an effort towards building a more inclusive future. 


\section{REFERENCES}

Ahmed, Sara. "Queer Feelings.” In the Cultural Politics of Emotion. Edinburgh: Edinburgh University Press, 2004.

Auslander, Philip. 2004. “I Wanna be Your Man: Suzi Quatro’s Musical Androgyny. “ Popular Music 23(1).

Banakou, Domna. 2010. "The Effects of Avatars' Gender and Appearance on Social Behavior in Virtual Worlds.” Journal of Virtual Worlds Research 2(5).

Bannister, Matthew. 2006. “'Loaded': Indie Guitar Rock, Canonism, White Masculinities.” Popular Music 25(1).

Barlett, Christopher P. and Richard J. Harris. 2008. "The Impact of Body Emphasizing Video Games on Body Image Concerns in Men and Women." Sex Roles 59(7/8):586601.

Bayton, Mavis. 1997. "Women and the Electric Guitar." Pp. 37-49 in S. Whiteley (Ed.), Sexing the Groove: Popular Music and Gender. New York, NY: Routledge.

Beasley, Berrin and Tracy Collins Standley. 2002. "Shirts vs. Skins: Clothing as an Indicator of Gender Role Stereotyping in Video Games." Mass Communication and Society 5(3): 279-293.

Behm-Morawitz, Elizabeth and Dana Mastro. 2009. "The Effects of the Sexualization of Female Video Game Characters on Gender Stereotyping and Female Self-Concept." Sex Roles 61(11/12): 808-823.

Berg, Bruce L. 2009. Qualitative Research Methods for the Social Sciences. $7^{\text {th }}$ Ed. Long Beacch, CA: California State University.

Berlant, Lauren and Elizabeth Freeman. 1992. "Queer Nationality." boundary 2, Vol. 19: $149-180$

Brookey, Robert A. and Kristopher L. Cannon. 2009. "Sex Lives in Second Life." Critical Studies in Media Communication 26(2):145-164.

Brooks, Daphne. 2008. "The Write to Rock: Racial Mythologies, Feminist Theory, and the Pleasures of Rock Music Criticism." Women in Music 12: 54-62.

Brown, R. Michael, Stephanie L. Brown, and Norma L. Brown. 1997. "Gender and Video Game Performance.” Sex Roles 36:793-812.

Bruno, A. (2009). "Gaming the System.” Billboard 121(3): 12. 
Bulik, B. S. 2007. 'Console Rock 'n' Roll: Two Video Games Clash.” Advertising Age 78(43): 8.

Bulik, B. S. 2008. "Video Games Rock the Music Industry." Advertising Age 79(26): 6.

Burgess, Melinda. C. R., Steven Paul Stermer, and Stephen R. Burgess. 2007. "Sex, Lies, and Video Games: The Portrayal of Male and Female Characters on Video Game Covers." Sex Roles 57(5/6): 419-433.

Butler, J. 1988. "Performative Acts and Gender Constitution: An Essay in Phenomenology and Feminist Theory." Theatre Journal 40(4): 519-531.

Butler, Judith. 1990. Gender Trouble: Feminism and the Subversion of Identity. New York, NY: Routledge

Butler, Judith. 1993. “Critically Queer.” GLQ. Volume I pgs. 17-32.

Butler, Judith. 1993. Bodies that Matter: On the Discursive Limits of "Sex." New York, NY: Routledge

Butler, Judith. 2004. Undoing Gender. New York, NY: Routledge

Cabiria, Jonathan. 2008. "Virtual World and Real World Permeability: Transference of Positive Benefits for Marginalized Gay and Lesbian Populations.” Journal of Virtual Worlds Research 1(1).

Churton, Mel and Anne Brown. 2010. Theory and Method. $2^{\text {nd }}$ edition. Basingstoke, UK: Palgrave.

Clawson, Mary Ann. 1999. "When Women Play the Bass: Instrument Specialization and Gender Interpretation in Alternative Rock Music." Gender and Society 13(2): 193-210.

Clawson, Mary Ann. 1999. "Masculinity and Skill Acquisition in the Adolescent Rock Band.” Popular Music 18(1).

Click, Melissa and Michael Kramer. 2007. "Reflections on a Century of Living:

Gendered Differences in Mainstream Popular Songs." Popular Communication 5(4).

Clifford-Napoleone, Amber R. 2015. Metal Bent: Queerness in Heavy Metal Music. New York, NY: Routledge.

Collins, Karen. 2013. Playing with Sound: A Theory of Interacting with Sound and Music in Video Games. Cambridge, MA: MIT Press. 
Connell, R.W. 1995. Masculinities. Los Angeles, CA: University of California Press.

Consalvo, Mia. 2012. "Confronting Toxic Gamer Culture: A Challenge for Feminist Game Studies Scholars.” Ada: A Journal of Gender, New Media, and Technology, 2012(1).

Crecente, B. (2007). “'Guitar Hero' still plucky.” Variety v. 408 no. 13 (November 12-18 2007) p. 51.

Croal, N. G. 2008. "They Put a Guitar Hero in Every House.” Newsweek 150(27/V151 part no1): 78-79.

Currah, Paisley and Lisa Jean Moore. "We Won"t Know Who You Are"e: Contesting Sex Designations in New York City Birth Certificates.” Hypatia 24, 3 (2009): 113-135.

Davies, Helen. 2001. "All Rock and Roll is Homosocial: The Representation of Women in the British Rock Music Press.” Popular Music 20(3).

Dahya, Negin, Jennifer Jenson, and Katrina Fong. 2017. “(En)gendering videogame development: A feminist approach to gender, education, and game studies." Review of Education, Pedagogy, and Cultural Studies,.39(4): 367-390

Den Tandt, Christophe. 2004. "From Craft to Corporate Interfacing: Rock Musicianship in the Age of Music Television and Computer-Programmed Music." Popular Music and Society 27(2): 139-160.

Dickerman, Charles, Jeff Christensen, and Stella Beatriz Kerl-McClain. 2008. "Big Breasts and Bad Guys: Depictions of Gender and Race in Video Games." Journal of Creativity in Mental Health 3(1).

Dietz, Tracy. L. 1998. "An Examination of Violence and Gender Role Portrayals in Video Games: Implications for Gender Socialization and Aggressive Behavior.” Sex Roles 38(5-6): 425-442.

Dill, Karen. E. and Kathryn P. Thill. 2007. "Video Game Characters and the Socialization of Gender Roles: Young People's Perceptions Mirror Sexist Media Depictions." Sex Roles 57(11/12): 851-864.

Donze, Patti Lynne. 2010. "Heterosexuality is Totally Metal: Ritualized Community and Separation at a Local Music Club.” Journal of Popular Music Studies 22(3).

Downs, Edward and Stacy L. Smith. 2009. "Keeping Abreast of Hypersexuality: A Video Game Character Content Analysis.” Sex Roles 62:721-733. 
Dozal, Mario A. 2016. "Consumerism Hero: The Selling Out of Guitar Hero and Rock Band." in Music Video Games: Performance, Politics, and Play. Michael Austin (Ed.). New York, NY: Bloomsbury.

Dibben, Nicola. 1999. "Representations of Femininity in Popular Music.” Popular Music 18(3): 331-355.

Dumitrica, Delia and Georgia Gaden. 2009) "Knee-High Boots and Six-Pack Abs:

Autoethnographic Reflections on Gender and Technology in Second Life.” Journal of Virtual Worlds Research 1(3): 3-23.

Eileraas, Karina. 1997. "Witches, Bitches, and Fluids: Girl Bands Performing Ugliness as Resistance." TDR 41(3).

Emerson, Rana. 2002. “'Where My Girls At?' Negotiating Black Womanhood in Music Videos." Gender and Society 16(1): 115-135

Entertainment Software Association (2017). "Essential Facts About the Video Game Industry." Retrieved from http://www.theesa.com/wpcontent/uploads/2017/04/EF2017_FinalDigital.pdf

Fast, Susan. 1999. "Rethinking Issues of Gender and Sexuality in Led Zeppelin: A Woman's View of Pleasure and Power in Hard Rock." American Music 17(3):245-299.

Feigenbaum, Anna. 2006. “'Some Guy Designed This Room I'm Standing In:' Marking Gender in Press Coverage of Ani DiFranco.” Popular Music 26(1).

Fox, Jesse and Jeremy N. Bailenson. 2009. "Virtual Virgins and Vamps: The Effects of Exposure to Female Characters' Sexualized Appearance and Gaze in an Immersive Virtual Environment." Sex Roles 61:147-157.

Fritz, B. 2007. “Heavy hitters tune up for rocker rivalry.” Variety 408(4): 1, 106.

Fritz, B. 2008. “'Hero's' beat goes on.” Variety 412(13): 45.

Fritz, B. and L. Alexander. 2008." 'Guitar' spinoffs play minor chord.” Variety v. 411 no. 8 (July 14-20 2008) p. 47.

Fullerton, Tracy, Janine Fron, Celia Pearce, and Jackie Morie. 2008. "Getting Girls into the Game: Towards a 'Virtuous Cycle.' In Beyond Barbie and Mortal Kombat: New Perspectives on Gender and Games. Cambridge, MA: MIT Press.

Gailey, Christine Ward. 1993. "Mediated Messages: Gender, Class, and Cosmos in Home Video Games.” Journal of Popular Culture. 27:81-93. 
Garr, Gillian. 1992. She's a Rebel: History of Women in Rock \& Roll. $2^{\text {nd }}$ ed.

Emeryville, CA: Seal Press

Geertz, Clifford. 1973. The Interpretation of Cultures. New York, New York: Basic Books, Inc.

Giddens, Anthony. 1990. Consequences of Modernity. Stanford, CA: Stanford University Press.

Giffort, Danielle M. 2011. "Show or Tell? Feminist Dilemmas and Implicit Feminism at Girls’ Rock Camp.” Gender and Society 25(5):569-588.

Goffman, Erving. 1959. The Presentation of Self in Everyday Life. New York, NY: Random House.

Goffman, Erving. 1977. "The Arrangement Between the Sexes." Theory \& Society 4(3): 301-331.

Groce, Stephen. B. and Margaret Cooper. 1990. "Just Me and the Boys? Women in Local-Level Rock and Roll.” Gender \& Society 4(2):220-229.

Hall Hansen, Christine. 1989. "Priming Sex-Role Stereotypic Event Schemas with Rock Music Videos: Effects on Impression Favorability, Trait Inferences, and Recall of a Subsequent Male-Female Interaction." Basic and Applied Social Psychology 10(4):371391.

Haraway, Donna. 1988. "Situated Knowledges: The Science Question in Feminism and the Privilege of Partial Perspective.” Feminist Studies 14(3): 575-599.

Harding, Sandra. 1986. "From the Woman Question in Science to the Science Question in Feminism." The Science Question in Feminism. New York, NY: Cornell University Press.

Harmonix Music Systems. http://www.harmonixmusic.com/about/timeline/

Haugen, Jason. 2003. “'Unladylike Divas': Language, Gender, and Female Gangsta Rappers." Popular Music and Society 26(4).

Hawkins, Stan. 1996. "Perspectives in Popular Musicology: Music, Lennox, and Meaning in 1990s Pop.” Popular Music 15(1):17-36.

Hayes, Elisabeth. 2005. "Women, Video Gaming \& Learning: Beyond Stereotypes." TechTrends 49(5). 
Hayes, Elisabeth. 2007. "Gendered Identities at Play: Case Studies of Two Women Playing Morrowind.” Games and Culture 2(1):23-48.

Heeter, Carrie, Rhonda Egidio, Punya Mishra, Brian Winn, and Jillian Winn. 2009. “Alien Games: Do Girls Prefer Games Designed by Girls?" Games and Culture 4(1):74100.

Hisama, Ellie. 2000. "Feminist Music Theory in the Millennium: A Personal History." Signs: Journal of Women in Culture \& Society 25(4).

Hubbard, Philip. 2000. "Desire/Disgust: Mapping the Moral Contours of Heterosexuality.” Progress in Human Geography 24(2): 191-217.

Jackson, Michele. 2007. "Exploring Gender, Feminism and Technology from a Communication Perspective: An Introduction and Commentary." Women's Studies in Communication 30(2).

James, Robin. 2009. “Autonomy, Universality, and Playing the Guitar: On the Politics and Aesthetics of Contemporary Feminist Deployments of the 'Master's Tools.'" Hypatia 24(2).

Jansz, Jeroen and Raynel G. Martis. 2007. "The Lara Phenomenon: Powerful Female Characters in Video Games." Sex Roles 56:141-148.

Jurgenson, Nathan. 2011. "Digital Dualism versus Augmented Reality." Cyberology: http://thesocietypages.org/cyborgology/2011/02/24/digital-dualism-versusaugmented-reality/

Kearney, Paul and Maja Pivec. 2007. "Sex, Lies, and Video Games.” British Journal of Educational Technology 38(3).

Kendall, Lori. 2000. "Oh No! I'm a Nerd! Hegemonic Masculinity in an Online Forum." Gender \& Society 14(2): 256-274.

Kennedy, Helen. 2002. "Lara Croft: Feminist Icon or Cyberbimbo? On the Limits of Textual Analysis." Games Studies 2(2).

Kirkland, Ewan. 2009. "Masculinity in Video Games: The Gendered Gameplay of Silent Hill.” Camera Obscura 24(2).

Kirkland, Ewan. 2005. "Restless Dreams in Silent Hill: Approaches to Video Game Analysis." Journal of Media Practice 6(3). 
Klastrup, Lisbeth and Susana Tosca. 2009. “'Because It Just Looks Cool!' Fashion as Character Performance: the Case of WoW." Journal for Virtual Worlds Research 1(3): 317.

Koerner, B. I. 2008. “It's OK to Party Like a Rock Star at Home.” Wired 16(2): 34, 38.

Laurel, Brenda. 2008. "Interview with Brenda Laurel (Purple Moon)." In Beyond Barbie and Mortal Kombat: New Perspectives on Gender and Games. Cambridge, MA: MIT Press.

Laurin, Helene. 2009. "The Girl is a Boy is a Girl: Gender Representations in the Gizzy Guitar 2005 Air Guitar Competition.” Journal of Popular Music Studies 21(3):284-303.

Leonard, David J. 2006. "Not a Hater, Just Keepin' It Real: The Importance of Race- and Gender-Based Game Studies." Games and Culture 1(1):83-88.

Lemish, Dafna. 2003. "Spice World: Constructing Femininity the Popular Way." Popular Music and Society 26(1).

Longhurst, Robyn. 1997. “(Dis)embodied Geographies.” Progress in Human Geography 21(4): 486-501.

Martins, Nicole, Dmitri C. Williams, Kristen Harrison, and Rabindra A. Ratan. 2009. "A Content Analysis of Female Body Imagery in Video Games." Sex Roles 61(11/12): 824836.

McClary, Susan. 1991. Feminine Endings. Minneapolis, MN: University of Minnesota Press.

McClary, Susan. 1993. "Reshaping a Discipline: Musicology and Feminism in the 1990s." Feminist Studies 19(2).

McClary, Susan. 2000. "Women and Music on the Verge of the New Millenium." Signs 25(4): 1283-1286.

McLeod, Ken. 2006. “'We are the Champions': Masculinities, Sports, and Popular Music." Popular Music and Society 29(5):531-547.

Mercer-Taylor, Peter. 1998. "Songs from the Bell Jar: Autonomy and Resistance in the Music of The Bangles." Popular Music 17(2):187-204.

Mettler, M. 2008. “Joe Perry of Guitar Hero." Sound \& Vision v. 73 no. 5 (June 2008) p. 88 .

Mikula, Maja. 2003. "Gender and Videogames: The Political Valency of Lara Croft." Continuum: Journal of Media \& Cultural Studies 17(1). 
Miller, Kiri. 2009. "Schizophonic Performance: Guitar Hero, Rock Band, and Virtual Virtuosity." Journal of the Society for American Music. (3)4: 395-429.

Miller, Kiri. 2012. Playing Along: Digital Games, YouTube, and Virtual Performance. New York, NY: Oxford University Press.

Miller, Monica K. and Alicia Summers. 2007. "Gender Differences in Video Game Characters' Roles, Appearances, and Attire as Portrayed in Video Game Magazines." Sex Roles 57: 733-742.

Morris, Rosalind. 1995. "All Made Up: Performance Theory and the New Anthropology of Sex and Gender.” Annual Review of Anthropology (24).

Mulvey, Laura. 1975/1999. "Visual Pleasure and Narrative Cinema." Film Theory and Criticism: Introductory Readings. Eds Leo Braudy and Marshall Cohen. New York, NY: Oxford University Press. 833-844.

Nakamura, Rika, and Hanna Wirman. 2005. "Girlish Counter-Playing Tactics.” Games Studies 5(1).

Nelson, Lise. (2014). "Engaging Butler: Subjects, cernment, and the ongoing limits of performativity." Performativity, Politics, and the Production of Social Space. 62-94.

Norris, Kamala O. 2004. "Gender Stereotypes, Aggression, and Computer Games: An Online Survey of Women.” CyberPsychology \& Behavior 7(6).

Nussbaum, Martha. 2000. “The Professor of Parody.” The New Republic Online.

O’Meara, Caroline. 2003. "The Raincoats: Breaking Down Punk Rock's Masculinities." Popular Music 22(3):299-313.

O’Meara, Daniel. 2016. "Rocksmith and the Shaping of Player Experience." in Music Video Games: Performance, Politics, and Play. Michael Austin (Ed.). New York, NY: Bloomsbury.

Ogletree, Shirley. M. and Ryan Drake. 2007. 'College Students' Video Game Participation and Perceptions: Gender Differences and Implications." Sex Roles 56(7/8): 537-542.

Pearce, Celia and Artemesia. 2009. Communities of Play. Cambridge, MA: MIT Press.

Pratt, Geraldine. 2004. "Spatializing the Subject of Feminism" in Working Feminism. Temple University Press. Pp. 12-37 
Reskin, Barbara F., Patricia A. Roos, Katharine M. Donato, Polly A. Phipps, Barbara J. Thomas, Chloe E. Bird, Linda A. Detman, and Thomas Steiger. 1990. Job Queues, Gender Queues: Explaining Women's Inroads into Male Occupations. Temple University Press.

Rheingold, Howard. 1993. The Virtual Community: Homesteading on the Electronic Frontier. Boston, MA: Addison-Wesley.

Roberts, Robin. 1990. “'Sex as a Weapon:’ Feminist Rock Music Videos.” NWSA Journal, 2(1).

Rodger, Gillian. 2004. "Drag, Camp, and Gender Subversion in the Music and Videos of Annie Lennox.” Popular Music 23(1):17-29.

Rosenberg, Jessica and Gitana Garofalo. 1998. "Riot Grrrl: Revolutions from Within." Signs 23(3).

Royse, Pam, Joon Lee, Baasanjav Undrahbuyan, Mark Hopson, and Mia Consalvo. "Women and Games: Technologies of the Gendered Self." New Media \& Society 9(4):555-576.

Salen, Katie and Eric Zimmerman. 2004. Rules of Play: Game Design Fundamentals. Boston, MA: MIT Press.

Sargent, Carey. 2009. "Playing, Shopping and Working as Rock Musicians: Masculinities in 'De-Skilled' and 'Re-Skilled' Organizations.' Gender \& Society 23(5).

Scharrer, Erica. 2004. "Virtual Violence: Gender and Aggression in Video Game Advertisements." Mass Communication \& Society 7(4).

Schiesel, Seth. 2009. "The Beatles Rock Band; All Together Now: Play the Game, Mom." The New York Times.

Schleiner, Anne-Marie. 2001. "Does Lara Croft Wear Fake Polygons? Gender and Gender-Role Subversion in Computer Adventure Games.” Leonardo 34(3).

Schilt, Karen. 2003. "A Little Too Ironic: The Appropriation and Packaging of Riot Grrrl Politics by Mainstream Female Musicians.” Popular Music \& Society 26(1):5-16.

Schippers, Mimi. 2000. "The Social Organization of Sexuality and Gender in Alternative Hard Rock: An Analysis of Intersectionality.” Gender \& Society 14(6): 747-764.

Sommers-Flanagan, Rita, John Sommers-Flanagan, and Britta Davis. 1993. "What's Happening on Music Television? A Gender Role Content Analysis.” Sex Roles 28(11/12). 
Shaw, Adrienne. 2009. "Putting the Gay in Games: Cultural Production and GLBT Content in Video Games." Games and Culture 4(3):228-253.

Sherman, Sharon. 1997. "Perils of the Princess: Gender and Genre in Video Games." Western Folklore 56(3/4).

Sioni, Sasha R., Mary H. Burleson, and Debra A. Bekerian. 2017. "Internet Gaming Disorder: Social Phobia and Identifying with Your Virtual Self." Computers in Human Behavior 71: 11-15.

SoundGirls.org.1993. http://www.soundgirls.org

Soukup, Charles. 2007. "Mastering the Game: Gender and the Entelechial Motivational System of Video Games." Women's Studies in Communication 30(2): 157-178.

Steinberg, Don. 2008. “Just Play.” Inc. Magazine.

Taylor, Nicholas, Jen Jenson, and Suzanne de Castell. 2009. "Cheerleaders/Booth Babes/Halo Hoes: Pro-Gaming, Gender, and Jobs for the Boys.” Digital Creativity 20(4).

Thornham, Helen. 2008. “'It's a Boy Thing:' Gaming, Gender and Geeks.” Feminist Media Studies 8(2).

Viamonte, Connie. 2015. "You Crit Like a Girl:' the Performance of Female Identity in the Virtual Gaming Community World of Warcraft." FIU Electronic Theses and Dissertations.

Wald, Gayle. 1998. "Just a Girl? Rock Music, Feminism, and the Cultural Construction of Female Youth.” Signs 23(3):585-610.

Walser, Robert. 1993. Running with the Devil: Power, Gender, and Madness in Heavy Metal Music. Hanover, NH: University Press of New England.

Watts, Evan. 2011. “Ruin, Gender, and Digital Games.” WSQ: Women's Studies Quarterly 39(3/4).

Weinstein, Deena. 2004. “All Singers are Dicks.” Popular Music and Society 27(3).

West, Candice and Don Zimmerman. 1987. "Doing Gender." Gender and Society 1(2): 125-151.

Westmoreland, Kalene. 2001. "“Bitch' and Lilith Fair: Resisting Anger, Celebrating Contradictions.” Popular Music \& Society $25(1 / 2)$. 
Whiteley, Sheila, ed. 2000. Women and Popular Music: Sexuality, Identity and Subjectivity. New York, NY: Routledge.

Winn, Jillian and Carrie Heeter. 2009. "Gaming, Gender, and Time: Who Makes Time to Play?" Sex Roles 61:1-13.

Wollman, Elizabeth. 1998. "Men, Music, and Marketing at Q104.3 (WAXQ-FM New York)." Popular Music and Society

Yao, Mike Z., Chad Mahood, and Daniel Linz. 2010. "Sexual Priming, Gender Stereotyping, and Likelihood to Sexually Harass: Examining the Cognitive Effects of Playing a Sexually-Explicit Video Game.” Sex Roles 62:77-88.

Yee, Nick. 2006. "Motivations for play in online games." CyberPsychology \& Behavior, 9(6): 772-775.

Yee, Nick. 2017. "Beyond 50/50: Breaking Down The Percentage of Female Gamers by Genre.” Quantric Foundry blog. January 29, 2017:

https://quanticfoundry.com/2017/01/19/female-gamers-by-genre/ 
VITA

ELISA M. MELÉNDEZ

Born, Rio Piedras, Puerto Rico

2006

B.A., Women's Studies

Florida International University

Miami, Florida

2009

M.A., Interdisciplinary Studies

Lesley University

Cambridge, Massachusetts

2014

M.A., Global and Sociocultural Studies

Florida International University

Miami, Florida

2013-2018

Doctoral Candidate

Florida International University

Miami, Florida

\section{PUBLICATIONS AND PRESENTATIONS}

Meléndez, Elisa (2013). "For Those About to Rock: Gender as Instrument in Rock Band" in Ctrl-Alt-Play: Essays on Control in Video Gaming; MacFarland Publishing, Matthew Wysocki (Ed.)

Meléndez, Elisa (April 2011). "For Those About to Rock: Gender as Instrument in Rock Band." Paper Presentation. Popular Culture Association National Conference; San Antonio, TX

Meléndez, Elisa (December 2010). "Chicks with Virtual Picks: Gender Performance on the Video Game Stage." Global and Sociocultural Studies Symposium. Florida International University; Miami, FL

Meléndez, Elisa (November 2010): "From Princesses to Frag Dolls: A Review of Women in Video Games." Poster Presentation; Women, Gender, Sexuality Studies Graduate Student Conference. Florida International University; Miami, FL

Meléndez, Elisa (October 2010). "Can One Play as a Guitar Heroine?: Music Performance as Gender Performance on the Video Game Stage." Southern Popular Culture Association/American Culture Association Conference; Savannah, GA 
Meléndez, Elisa (March 2009). "I'm in the Band:” A Course in the American Rock

Feminine. Paper presented at the Florida International University Women's Studies Student Conference. Miami, FL 